San Jose State University

SJSU ScholarWorks

Master's Theses

Master's Theses and Graduate Research

Spring 2014

\title{
Design of a Simplified Test Track for Automated Transit Network Development
}

Adam Livingston Krueger

San Jose State University

Follow this and additional works at: https://scholarworks.sjsu.edu/etd_theses

\section{Recommended Citation}

Krueger, Adam Livingston, "Design of a Simplified Test Track for Automated Transit Network

Development" (2014). Master's Theses. 4426.

DOI: https://doi.org/10.31979/etd.5tnx-gu6p

https://scholarworks.sjsu.edu/etd_theses/4426

This Thesis is brought to you for free and open access by the Master's Theses and Graduate Research at SJSU ScholarWorks. It has been accepted for inclusion in Master's Theses by an authorized administrator of SJSU ScholarWorks. For more information, please contact scholarworks@sjsu.edu. 
DESIGN OF A SIMPLIFIED TEST TRACK FOR AUTOMATED TRANSIT NETWORK DEVELOPMENT

\author{
A Thesis \\ Presented to \\ The Faculty of the Department of Mechanical Engineering \\ San José State University \\ In Partial Fulfillment \\ of the Requirements for the Degree \\ Master of Science
}

by

Adam L. Krueger

May 2014 
(C) 2014

Adam L. Krueger

ALL RIGHTS RESERVED 
The Designated Thesis Committee Approves the Thesis Titled

DESIGN OF A SIMPLIFIED TEST TRACK FOR AUTOMATED TRANSIT NETWORK DEVELOPMENT

by

Adam L. Krueger

APPROVED FOR THE DEPARTMENT OF MECHANICAL ENGINEERING

SAN JOSÉ STATE UNIVERSITY

May 2014

Dr. Burford Furman Department of Mechanical Engineering

Dr. Neyram Hemati Department of Mechanical Engineering

Dr. Ping Hsu Department of Electrical Engineering 


\title{
ABSTRACT \\ DESIGN OF A SIMPLIFIED TEST TRACK FOR AUTOMATED TRANSIT NETWORK DEVELOPMENT
}

\author{
by Adam L. Krueger
}

A scaled test platform has been developed for the purpose of testing, validating, and demonstrating key concepts of Automated Transit Networks (ATN). The test platform is relatively low cost, easily expandable, and it will allow future research and development to be done on control systems for ATN.

The test platform and reference vehicle were designed to adhere to a requirements document that was constructed to specify the necessary features of a fully functioning design. The work described in this thesis adheres to the phase one implementation of the requirements document. The vehicle and track were designed using commercially available computer aided design software. The prototype track was then assembled, and the vehicles were manufactured using a finite deposition three-dimensional printer. A control system was designed to control the velocity and position of the vehicle. This was accomplished using the feedback of a linear encoder that was designed and laid along the length of the track.

The vehicle functioned successfully according to the design requirements document. Testing showed that the vehicle is able to move to a specified position at a predetermined speed. Additionally, testing showed that a vehicle can maintain a specified following distance behind another vehicle within twenty millimeters. The vehicle and track can be used in the future to evaluate and validate specific questions regarding the implementation of an ATN system. 


\section{ACKNOWLEDGEMENTS}

This thesis is the result of the work of many individuals. I would first like to thank my committee Chair, Dr. Burford Furman, for his extensive support throughout the project. I would also like to thank my committee members, Dr. Neyram Hemati and Dr. Ping Hsu, for their input on this thesis. Jeff Davis from Lea+Elliott also provided a significant amount of support with the design requirements document, while gently guiding me in the correct direction, and pointing out roadblocks ahead. Lastly, I would like to dedicate this thesis to my family and friends for supporting me with in all of my pursuits. 


\section{TABLE OF CONTENTS}

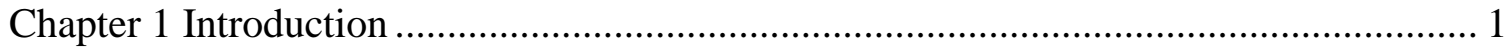

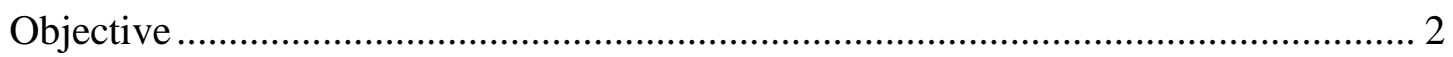

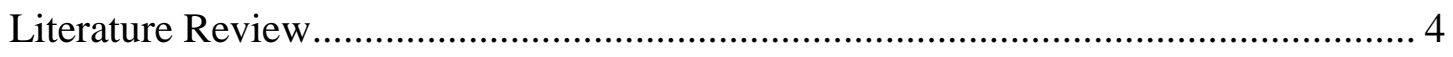

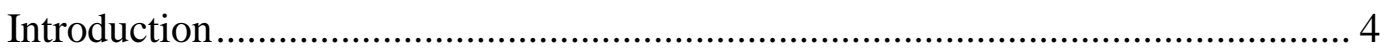

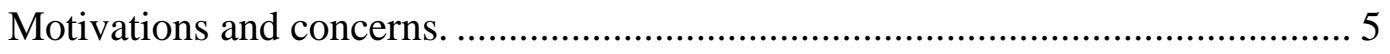

Features of ATN systems available ........................................................ 8

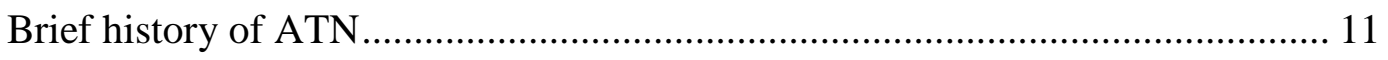

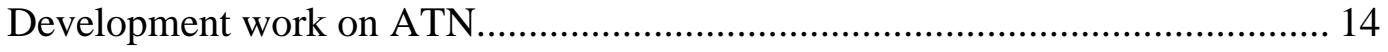

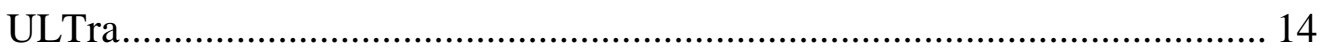

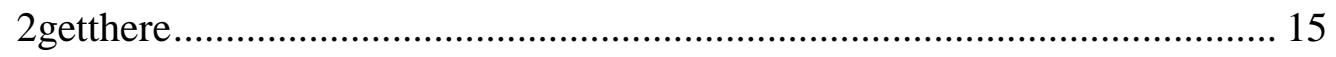

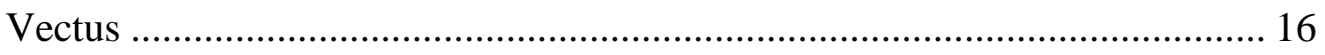

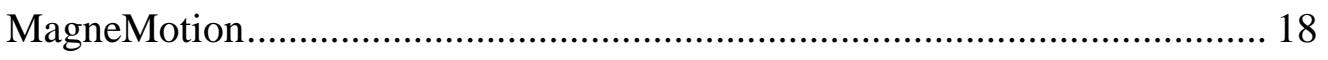

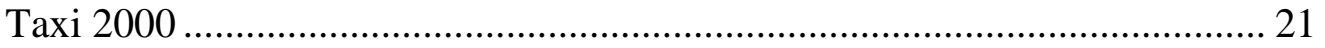

Aerospace Corporation ................................................................. 23

Conclusions and implications for this study ................................................. 25

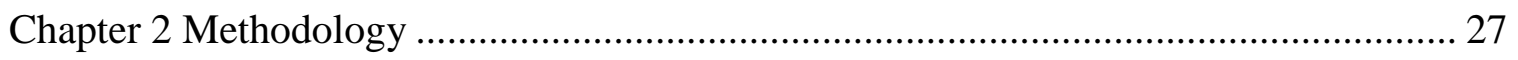

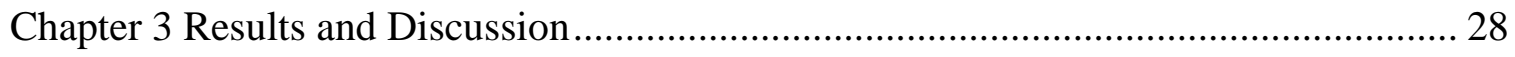

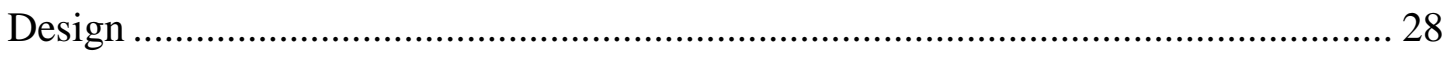

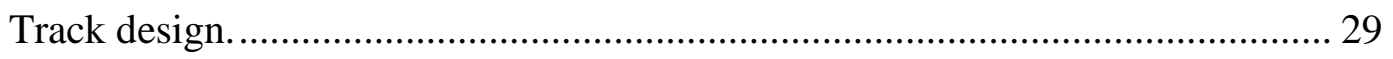

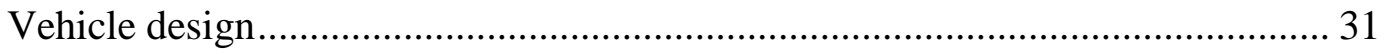

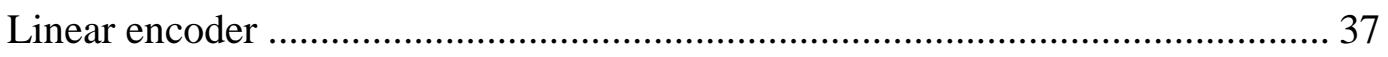




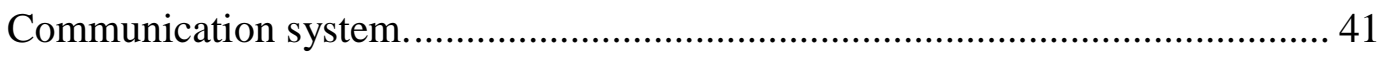

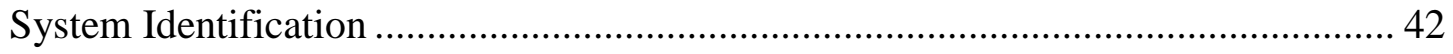

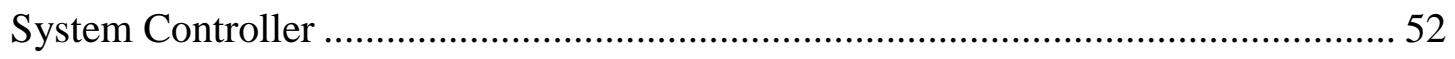

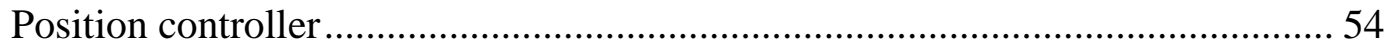

Cascaded position velocity controller ..................................................... 57

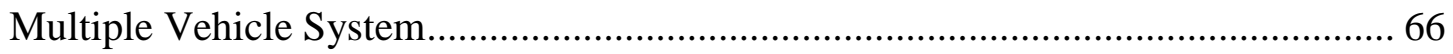

Chapter 4 Conclusions and Recommendations for Future Work .................................. 69

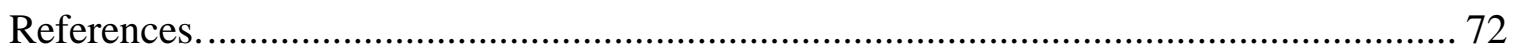

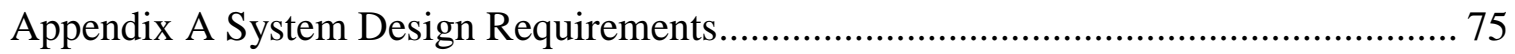

Appendix B Bill of Materials of the Track Assembly ............................................... 81

Appendix C Bill of Materials for the Vehicle Assembly .......................................... 82

Appendix D Drawings of Custom Parts................................................................ 83

Appendix E Matlab Proportional Controller Code .................................................... 97

Appendix F Matlab Cascaded Controller Code ....................................................... 98

Appendix G Microcontroller Code for the Vehicle .................................................. 99 


\section{LIST OF FIGURES}

Figure 1. Cabintaxi full-scale prototype system ................................................... 13

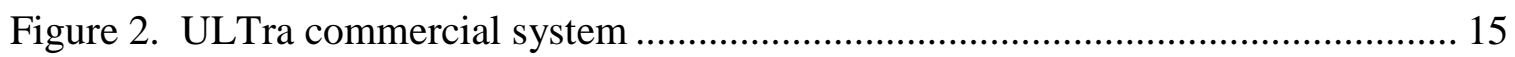

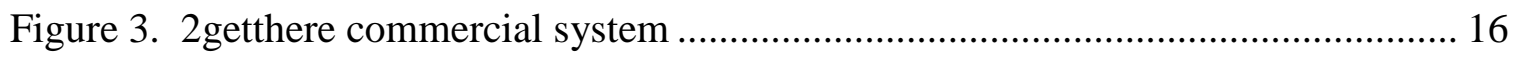

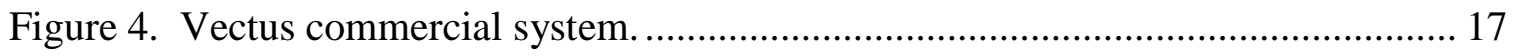

Figure 5. MagneMotion scale prototype ….......................................................... 19

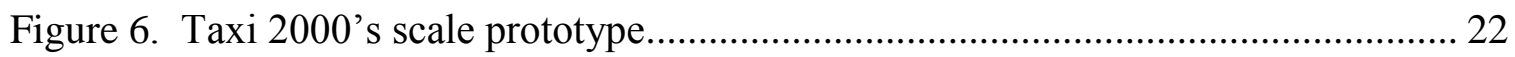

Figure 7. Aerospace Corporation's scale prototype ............................................... 24

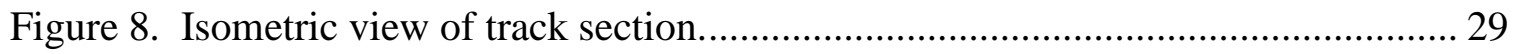

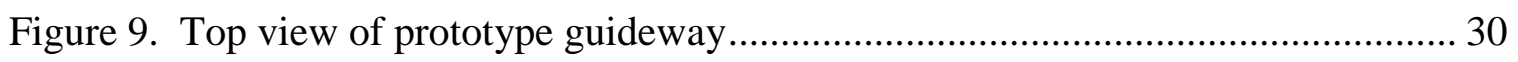

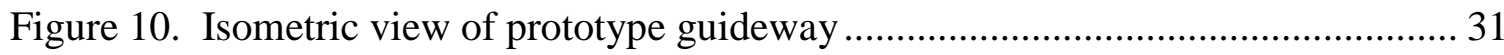

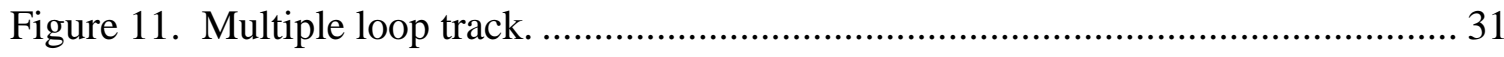

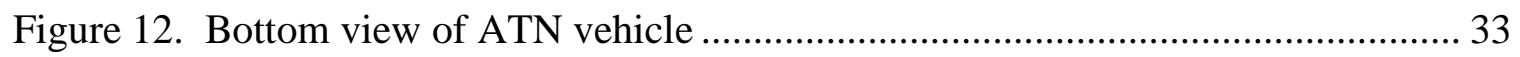

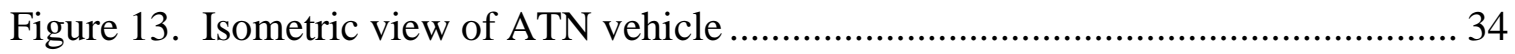

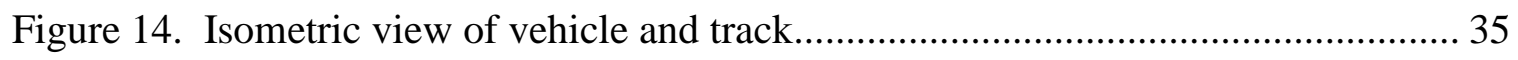

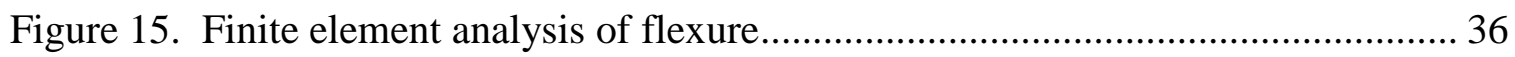

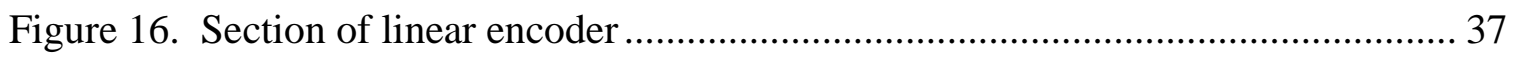

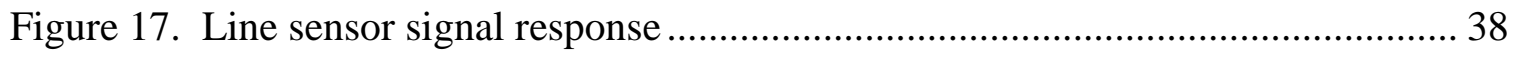

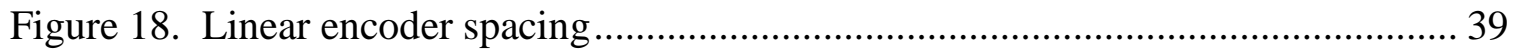

Figure 19. Quadrature encoder signal ............................................................ 41

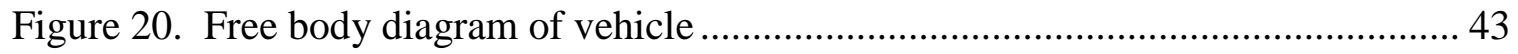

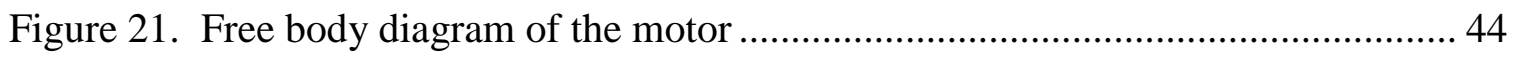


Figure 22. Electrical diagram of the motor.......................................................... 44

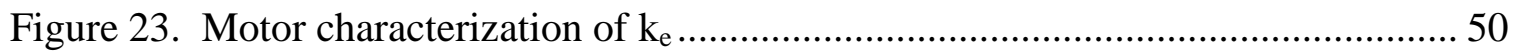

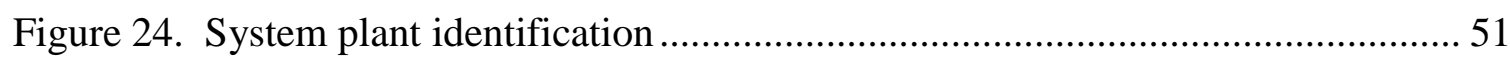

Figure 25. Block diagram of a system with a proportional gain controller ................... 52

Figure 26. Root locus of the open loop transfer function ....................................... 53

Figure 27. Step response of the vehicle with a proportional gain controller.................. 54

Figure 28. Block diagram of the system with a PID controller ................................... 55

Figure 29. Zeigler-Nichols criterion data for position control.................................. 56

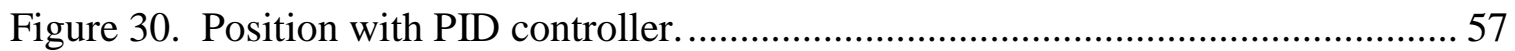

Figure 31. Velocity with a step voltage input ..................................................... 58

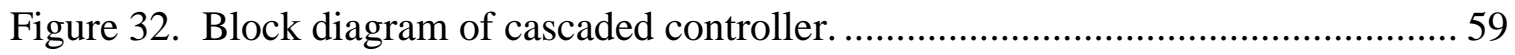

Figure 33. Zeigler-Nichols criterion data for velocity control................................... 60

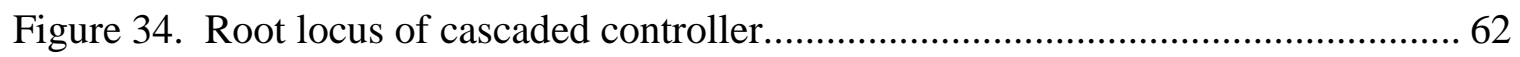

Figure 35. Closed loop pole and zero map of the cascaded controller......................... 63

Figure 36. Theoretical step response of the cascaded controller. ................................. 64

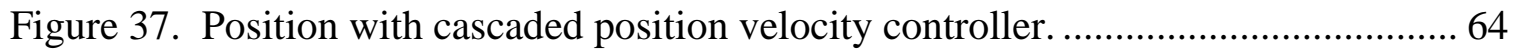

Figure 38 . Velocity with cascaded position velocity controller. ................................. 65

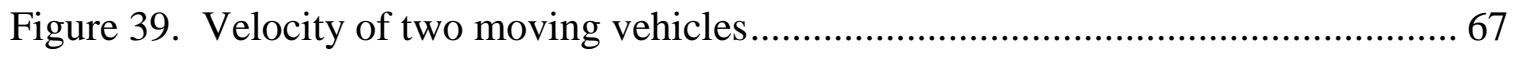

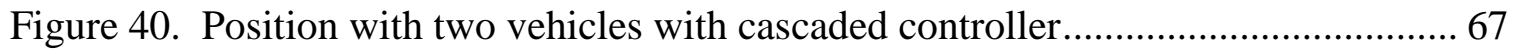

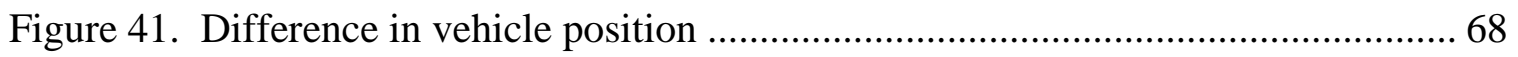




\section{Chapter 1 Introduction}

Transportation systems in use today are unsustainable. Most people drive automobiles as their current mode of transportation. There are inherent problems with using the automobile as a primary means of transportation in urban areas, which include limited fossil fuels, declining petroleum reserves, rising commute times, and growing pollution. Cities have tried to provide public transportation systems as alternative forms of transportation; however, the systems have not been widely adopted due to high costs, inconvenient schedules and coverage areas, and long commute times. Researchers at San José State University and in Sweden are working on solar-powered, Automated Transit Networks (ATN) as a potential solution to the problems with current transportation just described.

An ATN is a networked system of vehicles that operate autonomously on a dedicated guideway, which uses offline stations to carry passengers on demand from their origin to a specified destination. The concept has also been called Personal Rapid Transit (PRT) or pod cars. The system differs from conventional public transportation systems, such as light and heavy rail, because vehicles pick passengers up when they are requested, similar to a taxi, rather than on a set schedule as with conventional transit. On-demand travel allows the system to adapt to the passenger rather than the passenger adapting to the system. Additionally, the system transports passengers from their origin to their destination with no intermediate stops. An ATN is able to achieve this due to offline stations which allow mainline traffic to continue unimpeded. Additionally, vehicles in an ATN are designed to hold individuals or small groups of passengers who may wish 
to travel together rather than with strangers. This is not only an attractive way to travel for passengers, but it also allows for smaller, lighter vehicles that potentially could be powered electrically through solar photovoltaic panels mounted to the guideways and at stations. A system configured in this way would have the potential to capture enough energy to power the network within its footprint in an urban setting. Finally, most experts in the ATN field agree that the system should have a dedicated, elevated guideway used only for ATN vehicles. An elevated guideway provides several important benefits.

- Machines and humans are separated, which results in improved safety.

- Construction is less expensive compared to tunneling underground.

- Guideways can be placed in existing rights-of-way, such as roadways, to reach populated areas that have already been developed.

\section{Objective}

This thesis achieved the following objectives. First, simplified vehicles made of relatively inexpensive components were developed. In parallel, a test track was developed, which the vehicles travel on. The test track has four offline stations to demonstrate vehicle switching on diverging sections of track. These offline stations simulate passenger loading zones.

The system developed will continue to be used as an early stage prototype that can be used to test, validate, and demonstrate concepts used in ATN. As an example, the system can be used to validate various passenger loading scenarios, the effect of headway 
on capacity, and passenger wait time. The results of these studies will allow ATN to be better understood and will advance the state-of-the-art of ATN as a whole.

Important features of the system designed were that it was low cost, portable, easy to expand, and easy to assemble. It was necessary for the system to be low cost so that an expanded network could be built with modest funding. In this way, the network aspect of ATN with multiple vehicles and multiple stations could be investigated. An expanded network of guideways will enable investigation of system capacity, vehicle routing, empty vehicle management, and passenger surge loading before having to invest in a city-wide full-scale implementation.

The system should be portable so that it can be demonstrated to the public at places such as a city government center or library. This will facilitate educating the public about ATN and its benefits by showing how a model ATN system will look and how it will function.

The outcomes of the objectives described advance the state of the ATN by providing a platform where specific ATN concepts can be constructed, tested, and demonstrated. Currently, there are few small scale systems where ATN systems can be tested. There are many simulations of ATN systems, but there is little experimental data available. Additionally, most prototype systems are not small or accessible enough to be demonstrated to the public. The system described herein allowed experimental data to be collected and also allowed an ATN system to be demonstrated. 


\section{Literature Review}

Introduction. The idea for Automated Transit Networks has been around since at least 1953 (McDonald, 2013). Since then, many ATN systems have been conceived and designed including Cabintaxi, Vectus, Beamways, Ultra, H-bahn, and Skytran (McDonald, 2013). Of the many that have been designed, there are only a few that have been built and are functioning, which are Vectus, 2getthere, and ULTra. There are many reasons that ATN systems have taken this long to develop. This thesis will describe the state of development of ATN by first explaining the motivations and concerns related to ATN, detailing the important features of an ATN system, and providing the history of ATN. This background will give insight into the currently available ATN systems.

Next, the currently available scale prototype ATN systems will be discussed. Large scale commercial systems have still yet to be implemented even though ATN has come a long way with fully functioning systems. This is largely due to skepticism about the cost, return on investment, and functionality of a large capacity ATN system (Aerospace Corporation, 2012). Skepticism continues, even though there has been a substantial amount of theoretical and empirical work that demonstrates the functionality of ATN systems, as well as computer simulations that show the effectiveness of ATN systems. One way to overcome the skepticism is to install more ATN systems in order to validate the claims of ATN proponents. The advantage of model systems is that they demonstrate the effectiveness of an operational system without the need for as many simplifications needed for theoretical of simulation analyses. Prototype and model 
systems that have been developed will be described in detail so that future models can benefit from the triumphs and challenges of past work.

Motivations and concerns. ATN systems are often controversial and have become a topic of debate. A great deal of research has been conducted on the feasibility of the ATN systems, most of which has theoretically proven both the efficacy of the systems and its positive impact at alleviating congestion in highly populated areas. This research has recently led to the startup of many companies designing ATN systems and cities conducting studies on the feasibility of ATN systems including New Jersey (Carnegie \& Voorhees, 2007), Fresno, CA (Kimberly Horn and Associates, Inc., 2010), and San José, CA (Aerospace Corporation, 2012). Still, critics of ATN systems claim that ATN systems are unproven, costly, and too risky. Even the studies that have been performed for specific cities suggest that more research needs to be done of the topic before cities are willing to invest any money. As an example, San José paid for a feasibility study by Aerospace Corporation with the objective of performing a "rigorous, comprehensive analysis of the technology before determining whether to consider building a system" (Aerospace Corporation, 2012, p. 3). The results of the study stated that building an ATN system at the current time, in 2012, would be risky for the city because of the number of unanswered questions such as network capacity, power requirements, regulatory issues, and estimated cost. Similar results were found in studies performed in New Jersey stating that "PRT technology has not yet advanced to a state of commercial readiness" (Carnegie \& Voorhees, 2007, p. 5). As a direct result of these claims, more research and development is needed to address the unanswered questions. 
Specifically, research needs to be performed in the areas that these studies deem inadequate to reduce the risk associated with ATN systems.

If ATN can overcome the obstacles that are outlined in these studies, it has the potential to revolutionize transportation much in the same way the automobile did. John Anderson (2000), an expert in the field of ATN, provided a comprehensive list of the benefits of an ATN system in his review of ATN systems. Austin (2001) also described how ATN has the potential to revolutionize transportation in her description of idealistic transportation. A subset of the benefits is listed here.

- Fast, safe, private, secure, and all-weather transportation

- Reduction of roadway congestion

- Reduction accidents while travelling

- Reduction of air and noise pollution

- Reduction of energy usage

- Low street repair costs

- Improved mobility

In order for ATN to become a largely commercially based system, some skepticism with ATN systems needs to be overcome. The previous section alluded to some of the concerns with ATN. The concerns that were discovered by cities looking to implement ATN will be described below because these organizations are the ones that are looking seriously into building an ATN system.

- Average travel speed and overall trip times. Although there are many studies and simulations that have tried to describe the overall trip times, 
there are still questions about how this will work in practice. The major concern is that all of the studies that have been performed are conceptual (Carnegie \& Voorhees, 2007).

- System and station capacity. Capacity of stations and the total system continues to be a concern, even though theoretical capacities of 10,000 people per hour per direction can be achieved (Carnegie \& Voorhees, 2007). The main concern is how the system will respond in times of peak usage. Large wait times would minimize the effectiveness of the systems (Aerospace Corporation, 2012).

- Capital costs. The overall cost of the system is always a concern, especially when there is a large upfront cost that needs to be assumed by the public. There are preliminary estimates that predict ATN could cost approximately \$25 million per mile of guideway (Carnegie \& Voorhees, 2007).

- Operating and maintenance costs. There is a sizable risk in operating and maintenance costs required for an ATN system largely because these costs are contingent on a specific ATN design (Carnegie \& Voorhees, 2007). Additionally, costs associated with system outages are difficult to define.

- Energy use and environmental impact. The energy use of the system is contingent on the size of the system that will be implemented. This is largely affected by vehicle and guideway design that each individual 
system will use. Aerospace Corporation (2012) found that heating and ventilation concerns will be a large part of the power consumed.

Each of these concerns will need to be addressed in more detail for ATN to be more widely accepted. However, it seems that the major concerns with ATN are not that the system will not conceptually work. There are many papers that prove otherwise. The major concern is that there are few fully operational ATN systems available and the success of an ATN system is highly contingent on its final design. Therefore, many cities are hesitant to invest a large amount of capital with little knowledge of the final designs. More prototype systems, both small and full-scale, are needed in order to gain confidence in the concept of ATN as a whole.

Features of ATN systems available. The design of an ATN system is of the utmost concern when determining its effectiveness in a desired location. As a result, there are many important features that need to be incorporated into an ATN system for it to operate effectively and efficiently. These include capacity, switching, suspended versus supported vehicles, vehicle design, guideway design, reliability, safety, and energy considerations (Anderson, 2000).

Capacity is the single most important characteristic of ATN systems aside from safety for an effective ATN system. Capacity of the system directly affects the number of people the system can handle, wait times for passengers, and in turn, acceptance of the system. Capacity also has a large impact on the entire cost of the system. Larger vehicles can handle more passengers, which increases capacity; however, larger vehicles also require larger guideways to support the larger weight. Anderson (2005) describes 
how a system of many small vehicles versus a system with fewer larger vehicles with equivalent capacity will have a guideway weight and cost reduced by a factor of at least 20. Construction of guideways is the most expensive part of an ATN system (Anderson, 2000). Therefore, it is worth considering the possibility of using smaller vehicles. Smaller vehicles cannot carry as many people, so inherently the number of vehicles in operation would need to be greater. As a result, capacity and vehicle sizing are not trivial problems. Anderson (1984) has provided a detailed analysis of the capacity, cost, and size of traditional train systems versus an ATN system. He finds that it is cost effective to construct a properly designed ATN over a traditional bus transportation system. Still, the carrying capacity of each vehicle as well as the carry capacity of the system needs to be carefully considered for a system to be an effective transport method. Vehicle design is the second most important consideration of an ATN system. The vehicle design will dictate the capacity, the cost of the vehicle, as well as the cost of a guideway for it to run on. Furthermore, it has a large effect on the safety of the system and passenger comfort/acceptance. The ideal vehicle for a system will be relatively low capacity, low weight, and low cost. Approximately $90 \%$ of vehicles on the road during peak hours contain 1.2 persons (Anderson, 2000). Therefore, an ideal ATN vehicle would carry roughly the same amount of people. Additionally, research showed that the operation and maintenance costs are reduced if the smallest vehicles are used. As a result, special consideration should be given to the vehicle design to minimize size, weight, and cost. 
A switching mechanism is needed on the vehicle so that the vehicle can travel on a guideway that splits. Track splits are necessary so a vehicle can diverge off the guideway into a station or so that tracks that service different areas. The mechanism for which switching occurs has a great impact on the safety, reliability, and the capacity of the system. The switching mechanism that is used should be safe and repeatable. One of the considerations for a safe system is to have a locking system to ensure that the system cannot impale itself on a diverging section of track.

Combustion of hydrocarbon fuels is an increasing concern as global warming increases. For this reason, ATN should strive to minimize use of fossil fuels. Although the energy that is required for an ATN system is based on many factors, including vehicle design, propulsion system, guideway design and passenger loading, steps can be taken to minimize the energy consumption used in any system. The control system will have a large impact on the overall consumption of energy. For example, minimizing intermediate stops will greatly increase the overall efficiency of the system (Anderson, 2000). Regenerative braking can capture some of the energy normally lost during intermediate stops, but this is just a percentage of the energy needed to accelerate to operating speed. Additionally, the maximum acceleration needed in each vehicle will dictate the amount of energy the system consumes. "The maximum power requirement...can be cut almost in half with little penalty by gradually reducing acceleration above about half line speed" (Anderson, 2000, p. 15). As a result, special attention should be taken to evaluate the control systems that dictate intermediate stops, deceleration, and overall acceleration to minimize the energy usage of the system. 
Brief history of ATN. Numerous papers have been written examining the history of ATN, so that information from the past can be used to aid future development. McDonald's review gave a comprehensive review of ATN, including its origins. McDonald (2013) stated that the idea of ATN was conceived in 1953 independently by Donn Fichter and Ed Haltom. This information was supported by Anderson (2000) in his independent review of ATN. However, even though the idea of ATN was discovered in the early 1950s, research on the topic was largely un-collaborative until the US government endorsed the idea by passing the Urban Mass Transportation Act in 1964 (United States, 1964). Once the act was passed, there were many funded programs that sprung up leading to many research papers and company startups including Aerospace Corporation. Also, as a direct result of the federal funding surrounding ATN, the Morgantown ATN system was built (McDonald, 2013).

The Morgantown ATN system was funded and built because West Virginia University had limited space for the campus to grow while an increased number of students wanted to attend in the late 1960s. The solution was to build a separate campus 1.5 miles away. This split an already-separated campus into three campus locations. Congestion in the city quickly became a problem because of the increase traffic between campus locations. During this time, ATN was being worked on extensively, and it was considered as a concept to mollify the congestion problems. University officials proposed running a feasibility study on the concept of ATN in Morgantown. The feasibility study was conducted in 1970, which led to a federal grant for the construction of the system (Sproule \& Neumann, 1991). The system became operational carrying 
passengers on October 3,1975. This system was plagued with problems during the initial months of operation including vehicle malfunctions, sticking turnstiles, weather related problems, and exceeding the budget by a factor of four. Despite its initial setbacks, the system is still running today with an "operating reliability of over 99\%" (Sproule \& Neumann, 1991, p. 276).

The Aerospace Corporation (2014) had a large hand in getting the technology to a point that the Morgantown system could be built. It was set up after the Urban Mass Transportation Act of 1964 was passed as a non-profit entity and was funded by the government to develop technology of public importance. Jack Irving (1978) led the efforts of the company in developing the concept of an ATN system. They spent a large amount of time performing paper studies along with experimental design. The culminating project of the company was to develop a functioning prototype ATN system. After the company developed this, they published a book called Fundamentals of Personal Rapid Transit where they disclosed the lion share of work that the company had done with regard to ATN to spur public interest and share their knowledge with potential companies who would take the concept to a commercial state (Irving, 1978).

The surge of funding in ATN in the United States also spurred interest in other countries including Great Britain, Japan, Germany, France, Sweden, and Canada. Many companies spun out of the research performed in these countries with some large scale prototypes being constructed in countries such as Japan (McDonald, 2013).

The Cabintaxi program sprung out of international funding in Germany in 1969. The original development of the Cabintaxi system was started by two separate firms 
Messerschmitt-Bolkow-Blohm (MBB) and Demag. Then, in 1972, the German government facilitated combining the companies and funding the ATN project. The Cabintaxi system was developed as a result. The Cabintaxi system consisted of a twoway elevated track where one direction of the traffic moved above the guideway while the other traveled below the guideway. A full-scale test track was operational in 1976 with 24 vehicles and a 1.1 mile test track (Carnegie \& Voorhees, 2007). A picture of this is shown in Figure 1.

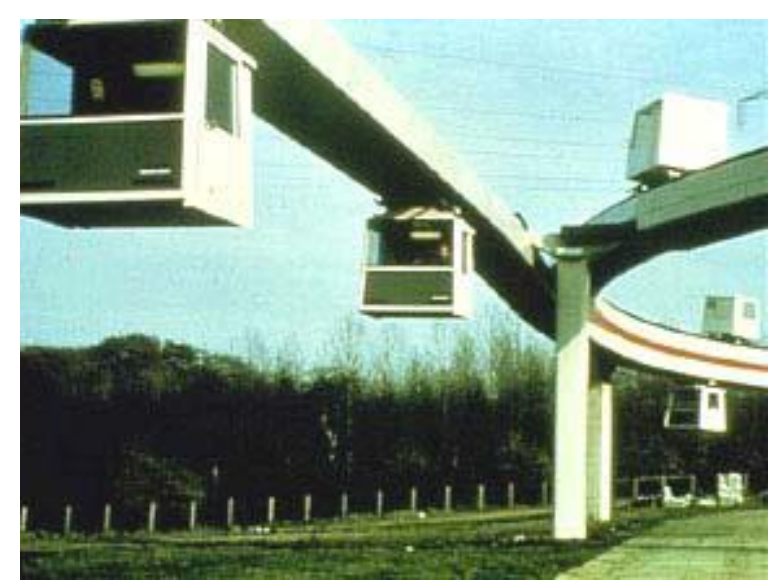

Figure 1. Cabintaxi full-scale prototype system (Carnegie \& Voorhees, 2007). Two-way traffic is achieved by having one traffic direction above the guideway and the opposite direction traffic below the guideway. Reprinted with permission from Cabintaxi.

Extensive testing was performed on this track, and it was studied expansively by outside firms interested in ATN, including many United States companies. There was a plan for a large scale system to be built in Hamburg, Germany, but lack of government funding led to the end of the program in 1980. A United States firm absorbed the technology, but the company is currently looking for funding to perform additional research and implement the technology at a commercial site. 
Over the next 50 years there have been many attempts at constructing a working ATN system. Today, there are several working ATN systems, although none of them are yet working under ideal conditions to realize the full benefits of ATN. The original concepts of ATN were ambitious and before their time. The technology needed to implement ATN was not available in the 1970s, making it expensive and difficult to design and construct a working system (Lowson, 2011). Still, the potential benefits of ATN have kept interest high in the technology. This can be seen in the large amount of funding for research that has cropped up over the years.

Development work on ATN. Research continues today on the concepts of ATN by both academia and private industries. There are many companies that have developed ATN systems that are waiting for potential sites to adopt them. These companies include MagneMotion Maglev, Vectus, Beamways, Ultra, H-bahn, 2getthere, and Skytran. ULTra, 2getthere, and Vectus have developed commercial systems and have implemented or are implementing them at specific sites.

ULTra. The ULTra system is operational in London, United Kingdom at the Heathrow airport. It began operation in 2010 with 2.4 miles of guideway, three stations, and 21 vehicles. The vehicles operate at a four second headway. This gives a one way capacity of 3600 seats per hour (Lowson, 2011). The guideway costs $\$ 15$ million/mile of one-way guideway (Helmer, 2009). This is less than the cost of developing a footbridge for walking pedestrians. The weight loading factor needed for pedestrians is $5000 \mathrm{~N} / \mathrm{m}^{2}$ whereas the loading factor needed for the ULTra systems is $2000 \mathrm{~N} / \mathrm{m}^{2}$ (Lowson, 2011). The system's vehicles are not track guided, but instead have a steering mechanism and 
four rubber tires. The system steers itself by using dead reckoning and sensors that relay to the control system the distance from the walls on the vehicle sides. The vehicle design is shown in Figure 2.

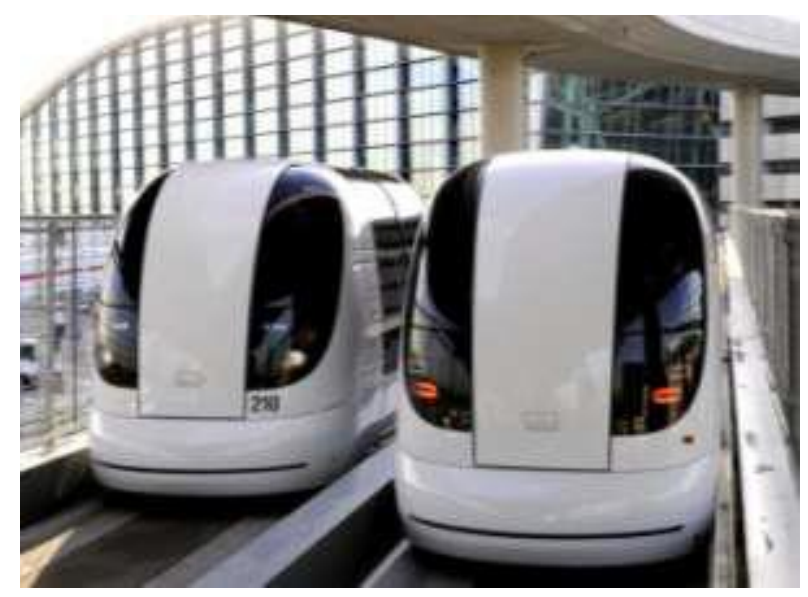

Figure 2. ULTra commercial system (Lowson, 2011). The system operates on an exclusive roadway with no rails. Reprinted with permission from ULTra.

2getthere. The 2getthere system is located in Masdar City, Abu Dhabi. It began operation in 2012 with 1.1 miles of guideway, five stations, and thirteen vehicles (2getthere, 2012). The current system is the link for the Masdar Institute of Science and Technology (MIST) (Muller, 2010). This is part of an initiative to make Masdar City the most sustainable city in the world. The city plans to not use any fossil fuel powered vehicles in the city. The plan is to eventually grow the ATN system to 3000 vehicles and 85 stations (2getthere, 2012). An example of a typical loading station is shown in Figure 3. 


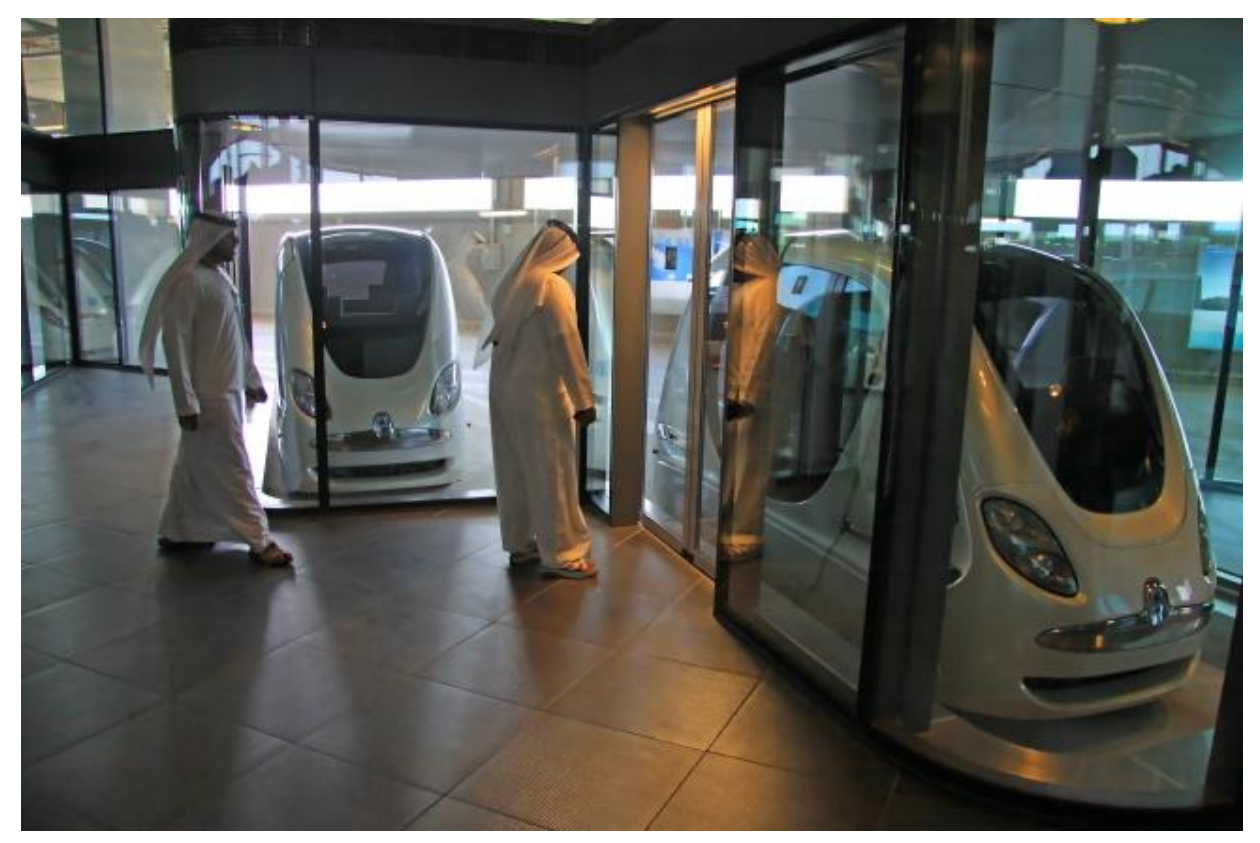

Figure 3. 2getthere commercial system (2getthere, 2012). Vehicle stations are shown where passengers can embark and disembark the vehicle. Reprinted with permission from 2getthere.

Vectus. Vectus is currently implementing a system in Suncheon Bay, South Korea. This system was expected to open in 2013 with 6 miles of guideway and 40 vehicles (Pemberton, 2013), but it is still undergoing system testing. The guideway is double tracked allowing two-way travel of vehicles along the guideway. A picture of the system is shown in Figure 4. 


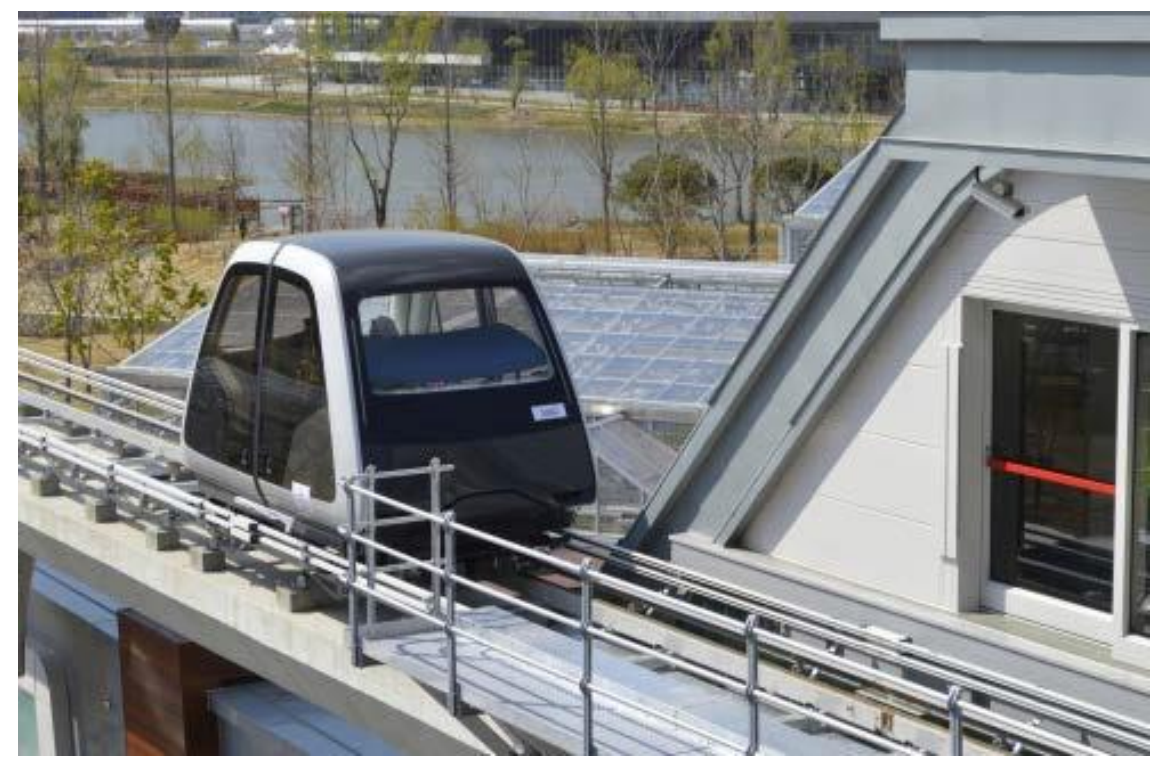

Figure 4. Vectus commercial system. The picture shows the double-tracked Vectus system in Suncheon Bay, South Korea. (Vectus Ltd., 2012). Reprinted with permission from Vectus.

The guideway being built has large pilings buried approximately 30 meters into the terrain due to the earthquake activity in the region. The system will start operation with two stations with the possibility of expansion. It is expected that the system will have 5000 passengers per day (Muller, 2010). The guideway is comprised of simple steel tubing. The mechanical switching mechanism is mounted in the vehicle, and is comprised of a wheel that moves to the outside of the diverging track, which guides the vehicle along the correct track (Muller, 2010).

The current systems that are available have limitations because they cannot scale to the capacity levels that the original promoters of ATN anticipated. Additionally, research is needed to prove that the capacities envisioned can be achieved. As a result, there is a need for robust scale models to further develop the technology. 
Prototype systems provide many benefits to learning about ATN systems. Simulations can provide intuitive information about how the systems will react. There are many simulations that are currently available that show how the control systems will work; however, simulations rarely provide a complete picture of the system. Instead, simulations use assumptions to simplify the system to make it easier to model. Assumptions still need to be made when assembling a prototype, but even a scale prototype will encounter similar disturbances that will be encountered in a full-scale system. As a result, a realistic system can be developed and tested for minimal costs compared to a full-scale system.

Many ATN developers have realized the benefits of developing prototype systems. The systems vary in size and scale. Companies such as MagneMotion, Vectus, Beamways, Ultra, Cabintaxi, Cabinlift, and Skytran have all built full size prototypes of their systems. There are also some ATN developers that realized the benefits of developing a scale system including MagneMotion, Taxi 2000, and Aerospace Corp. As described above, these small-scale systems are used to show operational systems, while not making unrealistic assumptions, for a fraction of the cost of a full-scale system or large-scale prototype. For this reason, the following section will describe each scale prototype system in detail, describe its advantages, and describe its disadvantages.

MagneMotion. MagneMotion developed a small test track system to showcase the development of their ATN concepts (Magnovate Technologies, 2013). The test track was mainly built to feature the effectiveness of their magnetic levitation system. The system used a Stabilized Permanent Magnet (SPM) suspension to levitate the 
MagneMotion vehicle. An additional highlight of the system is the track switching mechanism that MagneMotion uses. MagneMotion claims that this system is passive because there aren't mechanical or electrical components (Magnovate Technologies, 2013). In their prototype, the track switching is accomplished by manipulating the track stabilization fields at the diverging section of track. The field is manipulated to force the vehicle in the desired direction (Magnovate Technologies, 2013). The merging of the vehicle onto two converging tracks is handled automatically.

The system has one vehicle that moves on a test track with two switches via a linear synchronous motor (LSM). The system is shown in Figure 5.

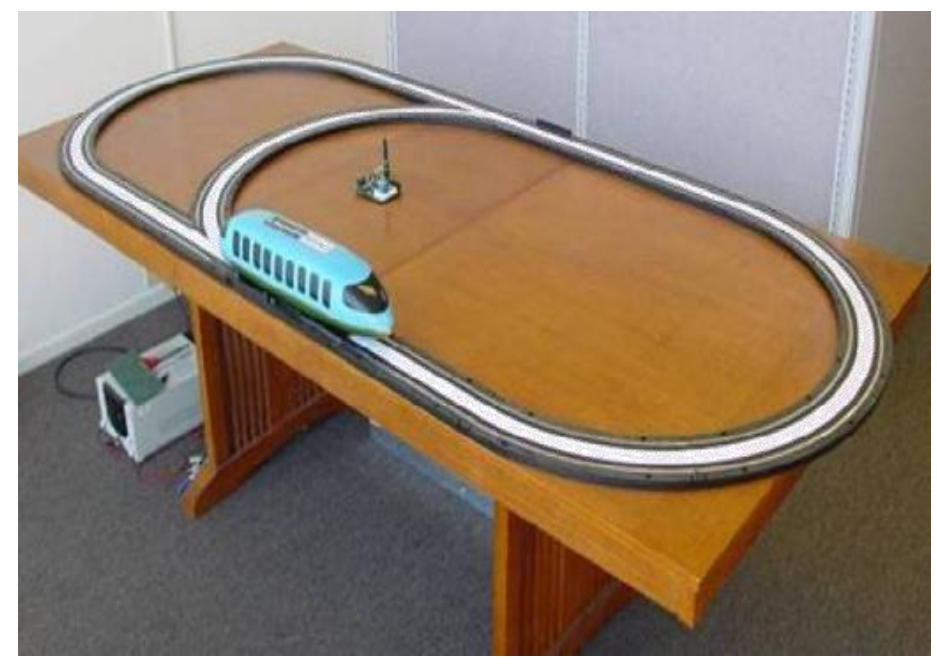

Figure 5. MagneMotion scale prototype. The system has a magnetic levitation ATN system with one vehicle (Magnovate Technologies, 2013). Reprinted with permission from Magnovate.

The vehicle shown has onboard radio control. It was designed to handle 20 to 40 passengers. MagneMotion expects that the vehicles could operate in platoons as well as individually. 
One of the main advantages of this system is that it effectively demonstrates the technology used behind both its drive mechanism and switching mechanism from track to track. A simulation would not intuitively explain the working mechanisms behind these systems. Furthermore, the system gives an effective representation of how the system will look because both the vehicle and the track were designed to look realistic from the outside. This is an important attribute because it shows the aesthetics of the system. Potential customers can easily get a feel for the aesthetic appeal of the system.

Although this prototype system is effective at showing the effectiveness of the magnetic levitation system, it lacks the capability to show how the system would react in many of the operating scenarios including multiple vehicle headways, fast track switching, multiple vehicle merges, and station overload.

One of the main attributes that people research in ATN is safe vehicle headway. Vehicle headway is a measurement of distance between two vehicles travelling at a velocity. Headway is measured by using the time it takes a trailing vehicle to be in the same position as the leading vehicle. A one vehicle system does not have the ability to show safe following distances can be achieved. If there were at least a two vehicle system, safe following distance could be experimentally measured. Additionally, tests could be conducted to determine how fast a vehicle could stop in an emergency.

Vehicles on ATN systems need to have a fast switching mechanism to quickly merge or diverge from tracks. For example, if vehicles were following in close proximity with a low headway, the system should be able to react fast enough so that vehicles do not miss diverging to a station or another guideway segment. The MagneMotion system 
does not have the ability to show the speed at which the track can switch to divert vehicles down multiple paths in real time. In theory, track switching such as this could be operated almost instantaneously; therefore, the system should not have any problem switching (Magnovate Technologies, 2013). However, it would be beneficial if this system had multiple vehicles so that it can show this.

Another main concern with regard to ATN is the ability to make safe merges on converging tracks. The MagneMotion system does not have the ability to show how multiple vehicle merges are performed. As a result, it cannot show how the control system will resolve potential merge conflicts when multiple vehicles want to merge at the same time. This could be improved by upgrading the prototype to have multiple vehicles.

Taxi 2000. Taxi 2000 (2010) developed a scale model of an ATN system that they named the Alpha Control System. The system is a 1/15th scale model comprised of a test track and twenty battery powered vehicles. The system uses a slot car approach to guide the vehicles on the track. In this method the vehicles roll along the track surface, but are guided by a slot cutout in the track. This slot allows the vehicle to only move in the direction of the slot. The vehicle movements are controlled via a master controlled that communicates to the vehicles through a wireless communication network. Proprietary TrakEdit software is used as the master controller. The vehicles can move at a maximum velocity of $0.7 \mathrm{~m} / \mathrm{s}$, which is the full-scale equivalent of $40 \mathrm{mph}$ (Taxi 2000, 2010).

The wood construction track has two loops and three stations on an elevated platform. The track is able to measure vehicle position using magnetic sensors embedded 
in the track to determine the absolute position of the vehicle. A picture of the system is shown in Figure 6.

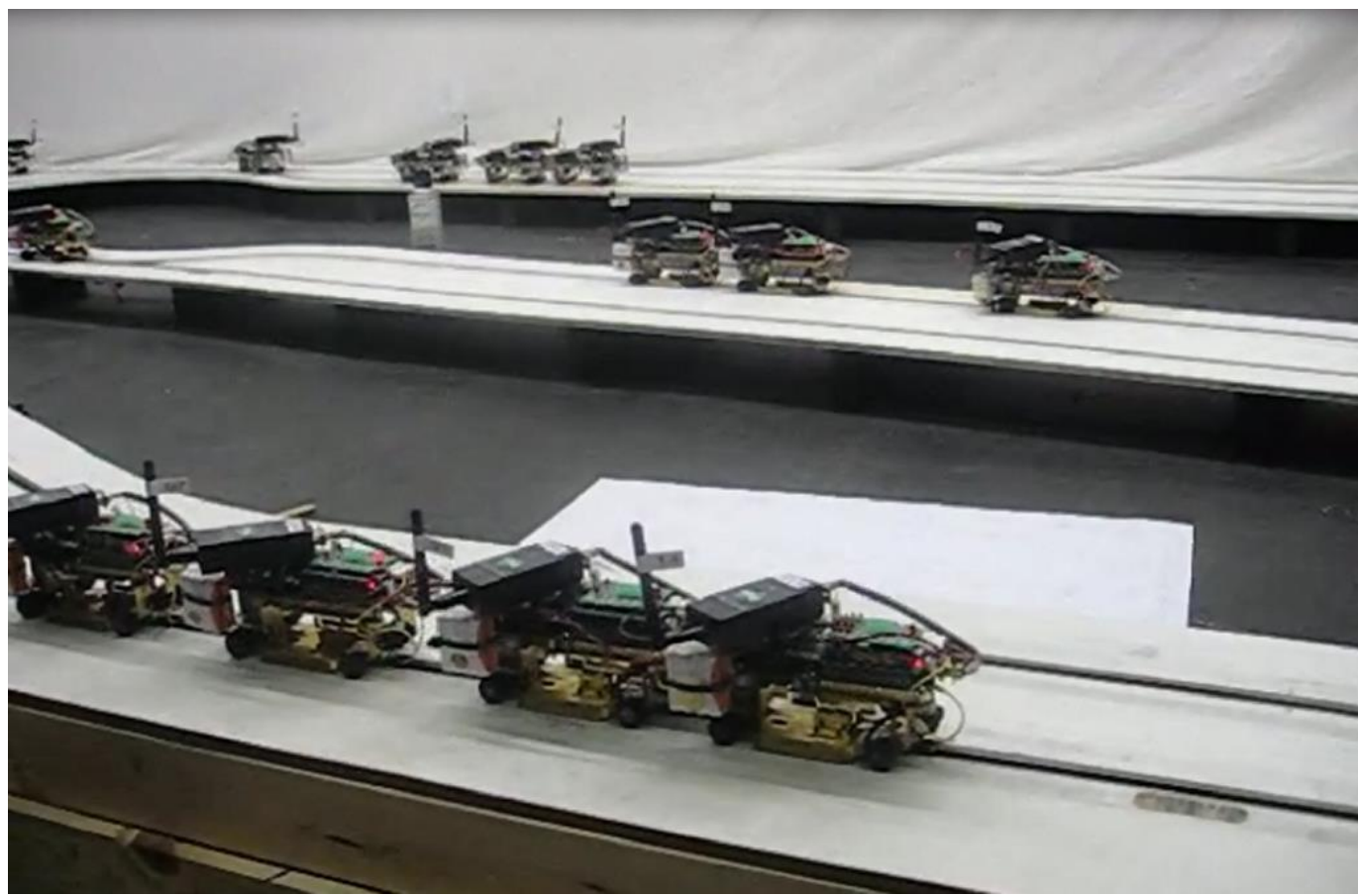

Figure 6. Taxi 2000's scale prototype. The company calls the prototype the Alpha Control System. It is a 1/15th scale personal rapid transit system (Taxi 2000, 2013). Reprinted with permission from Taxi 2000.

Some of the advantages of the system are that it can demonstrate multiple vehicle control with a specific headway, merging and diverging operations, and demonstrate system loading levels. These are all possible because the system has multiple vehicles and it has multiple stations for the vehicles to merge and diverge. In fact, this system was built to specifically test and validate some of the software schemes in the TrakEdit software (Taxi 2000, 2010). Validating the software was only possible by using multiple vehicles to demonstrate the various control schemas. 
The Alpha Control System had a few disadvantages, including lack of passenger information, lack of a realistic guide way, and overall cost. The system does not have the ability to display passenger information at the stations. Since passenger movement is at the heart of ATN, it would be beneficial to show the movement of passenger from stations to vehicles, and then to destination stations. Passenger indicators would make it easier to visualize the movement of passengers. Additionally, the system could be used to demonstrate the movement of empty vehicles to fill current demand at stations as well as movement of vehicles to fulfill predicted future demand.

Second, the system is costly. The system is extensive, with twenty vehicles, and each of these vehicles costs money. Additionally, the track is costly to assemble because there is a significant amount of time invested in cutting the track pieces and assembling them.

Aerospace Corporation. The Aerospace system was designed and built in 1971. It was one of the first scale models built to show the concept of ATN. The concept was a $1 / 10^{\text {th }}$ scale model consisting of a $45 \times 14$ foot oval track and three vehicles (Irving, 1978). The vehicles operated using linear electric motors that were onboard each of the vehicles. The nominal vehicle velocity was $3 \mathrm{ft} / \mathrm{s}$ which corresponded to a full-scale velocity of $20.5 \mathrm{mph}$. All the propulsion systems in the model were scaled directly to $1 / 10^{\text {th }}$ tenth the size of a full-scale system. Also, the dimensions of the motors and track and the motor power were scaled exactly to $1 / 10^{\text {th }}$ of a full-scale system. The mass of the system was scaled appropriately to account for the inertia of the system when 
accelerating. In this way, the propulsion system was designed so that it would mimic a full size model accurately. A picture of the prototype system is shown in Figure 7.

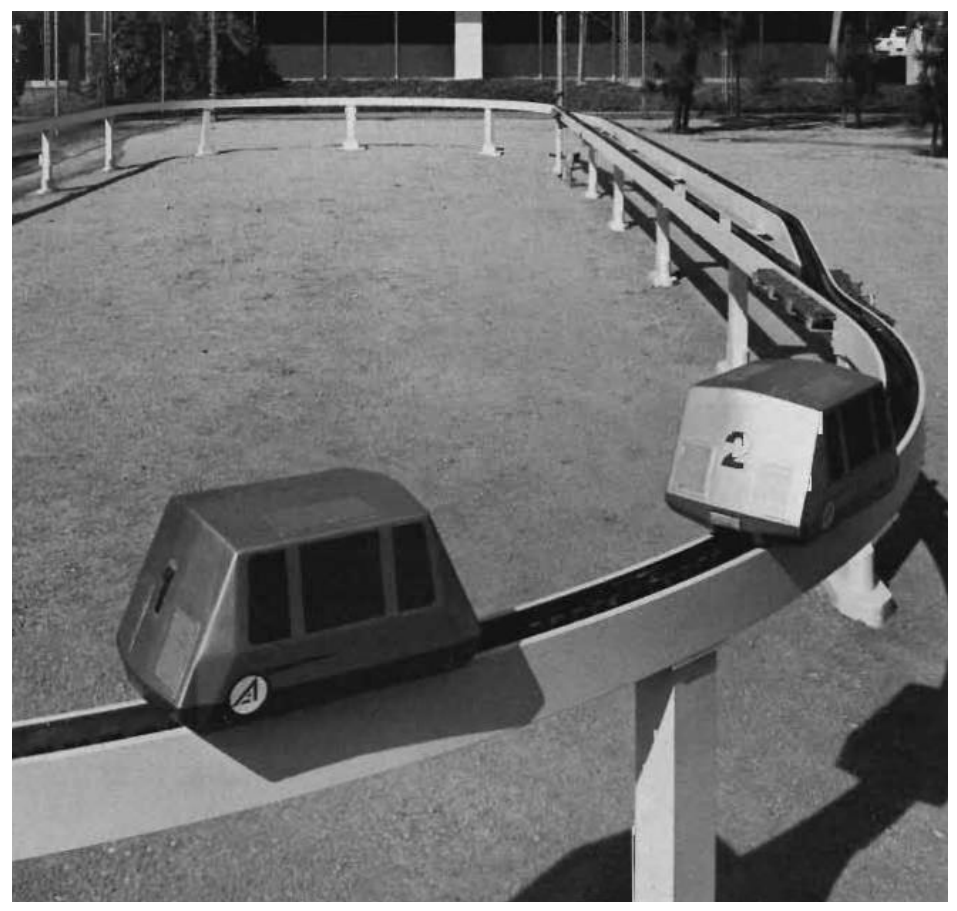

Figure 7. Aerospace Corporation's scale prototype (Irving, 1978). This is a $1 / 10^{\text {th }}$ scale prototype with three vehicles. Reprinted with permission from Aerospace Corporation.

The main advantage of this system was the effort that was put into sizing the system appropriately in all facets including size, weight, and propulsion. This accurate sizing of the system allowed each subsystem to be tested effectively without making large assumptions. Additionally, the vehicles were designed to look similar to a final concept. As described above, this showcases the aesthetic appearance of the vehicles.

The outdoor nature of this system also allowed it to be tested against the elements in a similar situation to how a commercial system would have to perform. Again, this allowed for thorough testing to be conducted on the prototype in many different types of conditions. 
One of the main disadvantages of this system is its size. The $1 / 10^{\text {th }}$ scale model would require ample installation space as well as a large amount of money to fully implement. Interestingly, Aerospace Corp. selected the scale based on the technology that was available at the time. Aerospace Corp. did not think that they could effectively house all the electronics in the vehicles at a smaller scale. Electronics have decreased orders of magnitude in size since the 1970s. Therefore, a smaller scale would not be a problem for scale models built today.

Another disadvantage of the system is that it only has one station on the guideway and only three vehicles. A multiple station prototype has the ability to show how the vehicles could be used to transport passengers throughout the system without excessive delays. Demonstration on the movement of empty vehicles is also necessary to show how the vehicle will respond to unbalanced demand. This would be difficult to do with only three vehicles and one station.

Conclusions and statement of problem. ATNs have many benefits that can be realized with a properly developed system. Urban traffic congestion, dependence on fossil fuel, and reduced emissions are a few of the direct benefits that can be realized from ATN in addition to many of the indirect benefits that can provide value to a city. With all these benefits, only a few cities have adopted ATN systems and most of them have happened only recently. Feasibility studies have shown that most cities have not adopted ATN systems because they will be assuming too much risk. The studies claim that the technology is still too immature to risk the capital necessary to implement them. The current system implementations will help other cities adopt ATN systems as they 
observe the effectiveness of the ATN implementations. In addition, more research needs to be performed on key areas of ATN to minimize the risk that interested cities will have to assume.

Theoretical research as well as conceptual research needs to be performed. Unfortunately, there are few good physical models of systems that have been implemented. Implementation of a physical model is important because it can demonstrate the important features of ATN with a low up front cost. It also provides an avenue for interested parties to experiment and develop ATN technology further. With an effective model, many of the benefits of ATN can be discovered, and many of the problems will be exposed, allowing the world to come closer to an environmentally responsible, economical solution to transportation that passengers will appreciate.

Even though there are a limited number of scale models, the few shown here offer insight into the important elements of a scale model. The scale vehicle needs to be small, easy to manufacture, and minimal cost. These attributes are essential because a scale system needs to have at least three vehicles to accurately model an ATN system. A system with fewer vehicles makes it difficult to simulate ATN systems. The Taxi 2000 model shows the benefits of having a complex system.

Additionally, the track needs to be minimal cost, easy to manufacture, and easy to assemble in custom configurations. An important feature of a scale ATN track is that it can be configured similar to a full-scale system. As a result, it is important that the track can be easily constructed to mimic full-scale implementations. Also, the ability to quickly expand the track and vehicle system will allow more complex systems to be 
tested. Therefore, special care needs to be taken to quickly and easily expand the track and size of the vehicle fleet.

\section{Chapter 2 Methodology}

Prototype systems are necessary to show the development of the design concept. There are many steps in the design process that are listed below.

1. Concept

2. Design requirements and design specifications

3. Scale model (functional)

4. Full-scale mock-up (non-functional)

5. Full-scale engineering development prototype

6. Final design

There are few ATN prototype systems that are currently available in the functional scale model step. One of the reasons for this is that there has been funding available for the development of full-scale prototype systems in the past. However, scale models are more effective at building confidence to get additional funding when working with a limited budget (Transport Innovators, 2013). Still, experts studying ATN have noted that there is a lack of acceptable scale ATN models. Therefore, a low cost system that can be used to successfully test, validate, and demonstrate various ATN control concepts was developed.

In order to design a system that meets all the requirements needed for an effective ATN model, a design requirements document was developed and it is attached to the end 
of this document in Appendix A. This document outlines the necessary requirements for the system to work properly. These requirements were necessary because they helped to determine what features were needed in the design. This guided the design to ensure the

final system functioned as it was intended. Once this document was complete the design of the system commenced.

One of the requirements of the system was for it to be track guided and not independently steered. The reason for this was that it was important for the system to be similar to a track guided system that has track switching control. As one might expect, track switching controls could not be developed without a track guided system.

Another requirement was to design a system that approximates a currently available system. This allows testing and validation of controls schemes on a model level. The system design was chosen to approximate the Vectus system and other real systems. Therefore, required features of the design were that the vehicle is supported underneath, it has guide wheels along the track, and it has a mechanically operated switching mechanism to guide the vehicle along a diverging section of track.

\section{Chapter 3 Results and Discussion}

\section{Design}

In the following section, the design of the prototype ATN system is described in detail. Additionally, a supplementary video of the system was made so that the dynamics of the system could be better understood. This video was then posted to the internet and can be viewed by referring to the web site titled "Automated Transit Network (ATN) 
prototype" posted in the references of this document (Krueger, 2014). Also, drawings of the custom parts are included in Appendix D for reference.

Track design. Many ideas were developed in the process of designing an economical, easy to assemble track. The most economical system was constructed of bent sheet metal strips mounted to a plywood board with screws. This design is shown in Figure 8 .

(a)

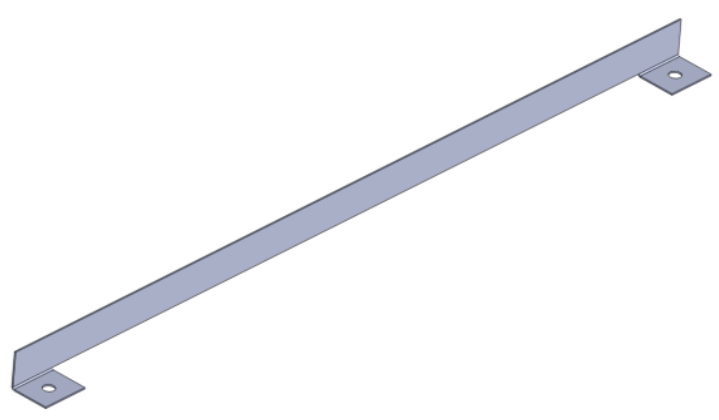

(b)

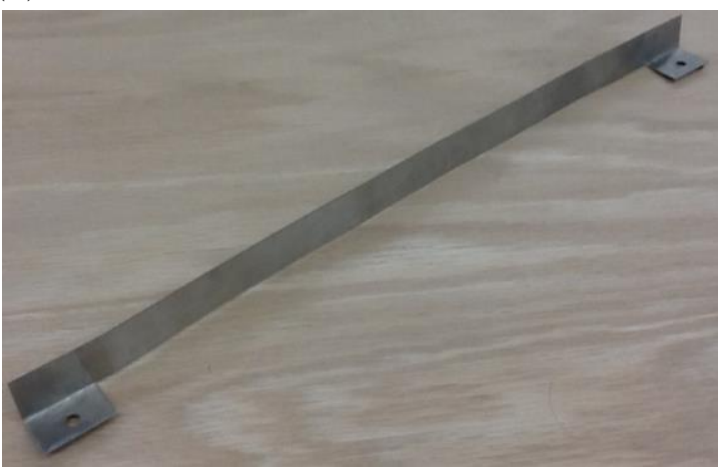

Figure 8. Isometric view of track section. The sheet metal part shown forms a vertical section of track that the vehicle can ride along. The 16 in long part is bolted to plywood with two tabs that have a hole punched in them.

The overall cost of the track is shown in the bill of materials for the track in Appendix B. The track guideway has four stations designed to fit in an $8 \times 8$ foot area. The station locations are shown in Figure 9. 


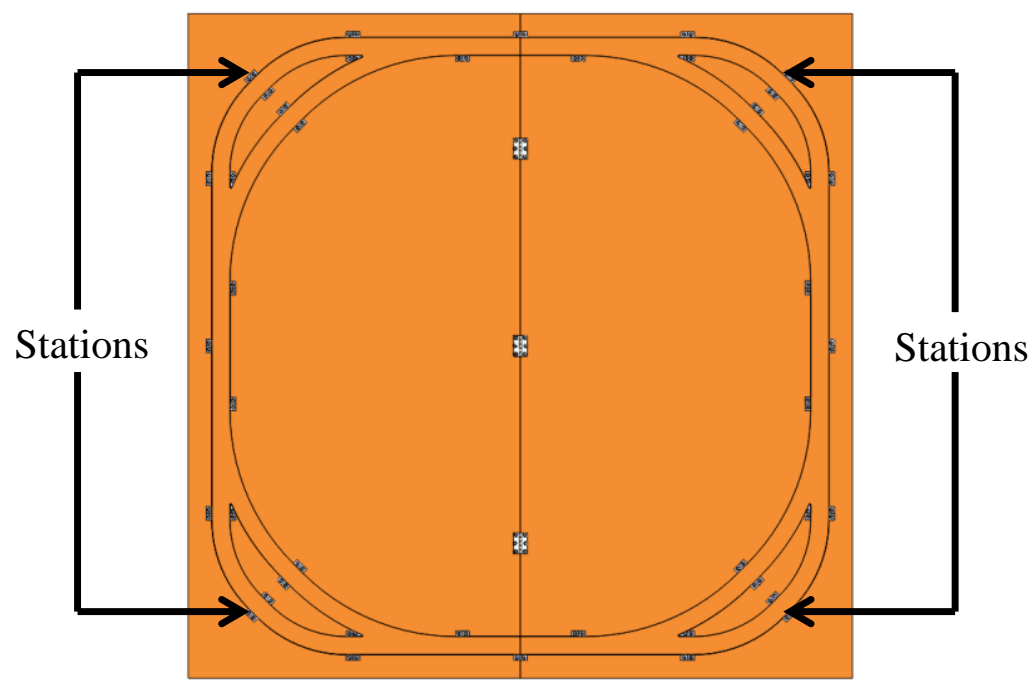

Figure 9. Top view of prototype guideway. The guideway is mounted on two plywood sheets with four stations with one at each of the four corners.

Four stations were incorporated into the track to allow each vehicle to have three alternate stations where it could travel. A four station system is sufficiently complex so that the routing algorithms used by each car are not trivial. The final guideway is shown in Figure 10. 
(a)

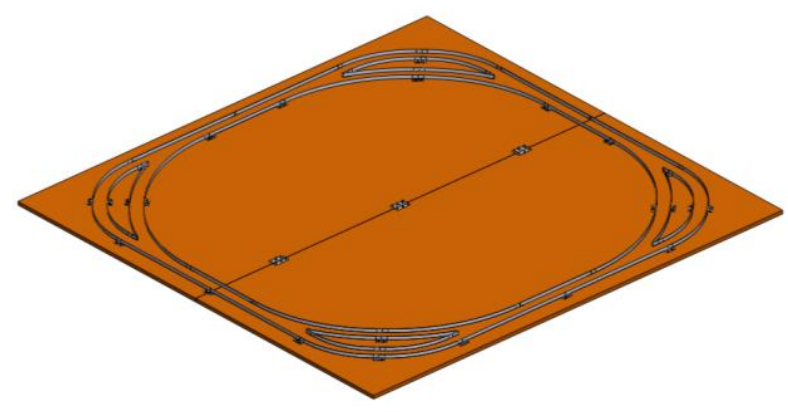

(b)

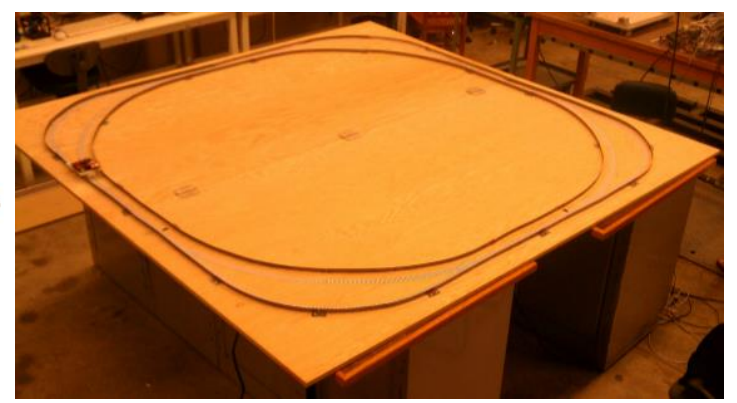

Figure 10. Isometric view of prototype guideway. The guideway consists of vertical sheet metal strips that are mounted to plywood by bent tabs.

The track system can be expanded quickly and inexpensively by creating a diverging section of track that moves to a new area. One example of this is shown in Figure 11, where an addition loop is added to the existing track.

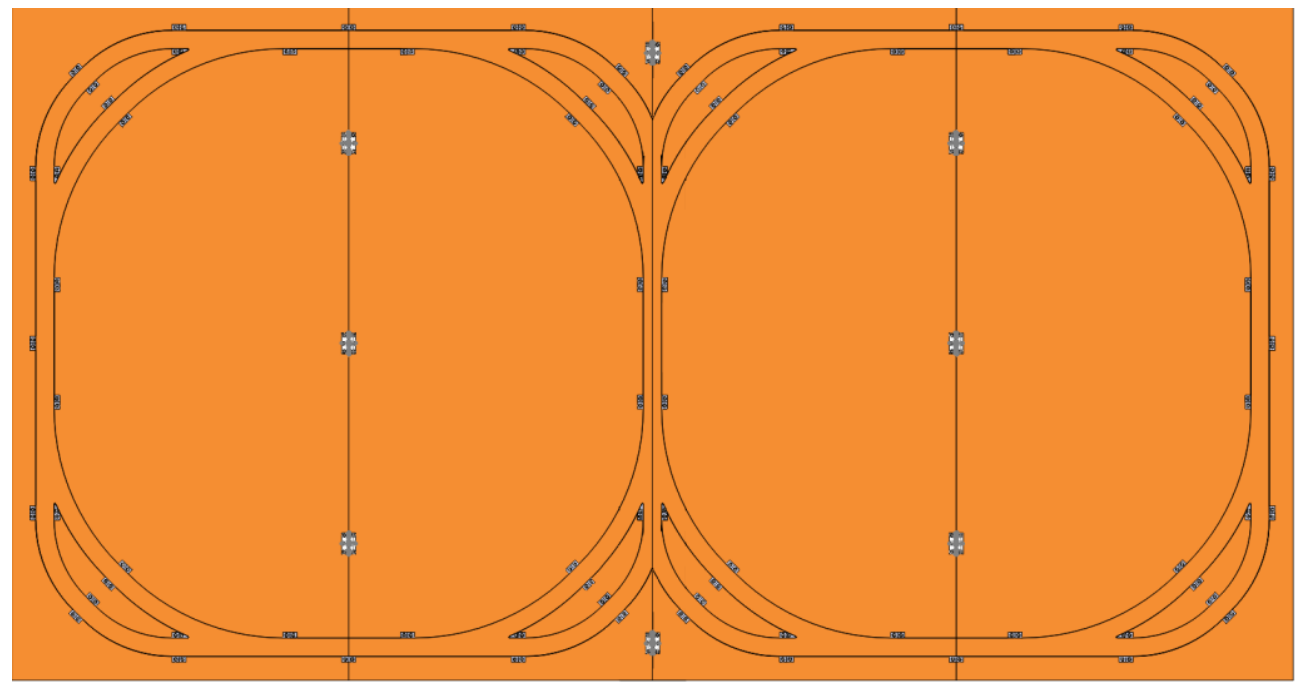

Figure 11. Multiple loop track. The picture shows an alternate setup of the track where an additional diverging section of track can be added to expand the track system.

Vehicle design. The vehicle was designed to be able to traverse the guideway smoothly while having adequate sensors incorporated to control the vehicles. 
Additionally, the chassis was designed to be inexpensive to minimize costs if the system is expanded. In order to minimize cost, the chassis was designed around 1/32 scale slot car parts because these parts are readily available and inexpensive. In order to quickly fabricate the vehicle, a Stratasys uPrint SE Finite Deposition Model three-dimensional printer was used. The material used was white acrylonitrile butadiene styrene (ABS) plastic. The overall size of the vehicle is $94 \times 140 \times 50 \mathrm{~mm}$ and the overall cost of the vehicle is approximately $\$ 157$ and the cost breakdown is shown in Appendix C.

The vehicle is powered by three 3.7 volt lithium ion batteries. These batteries are connected in series to provide power at 11.1 volts to both an Arduino Uno R3 microcontroller and an SN754410 half H-bridge driver. The system is propelled by a Scalextric C8146 $12 \mathrm{~V}$ direct current (DC) motor. The drive motor is connected to the rear axle by a 3:1 gear ratio. This is shown in Figure 12. 
(a)

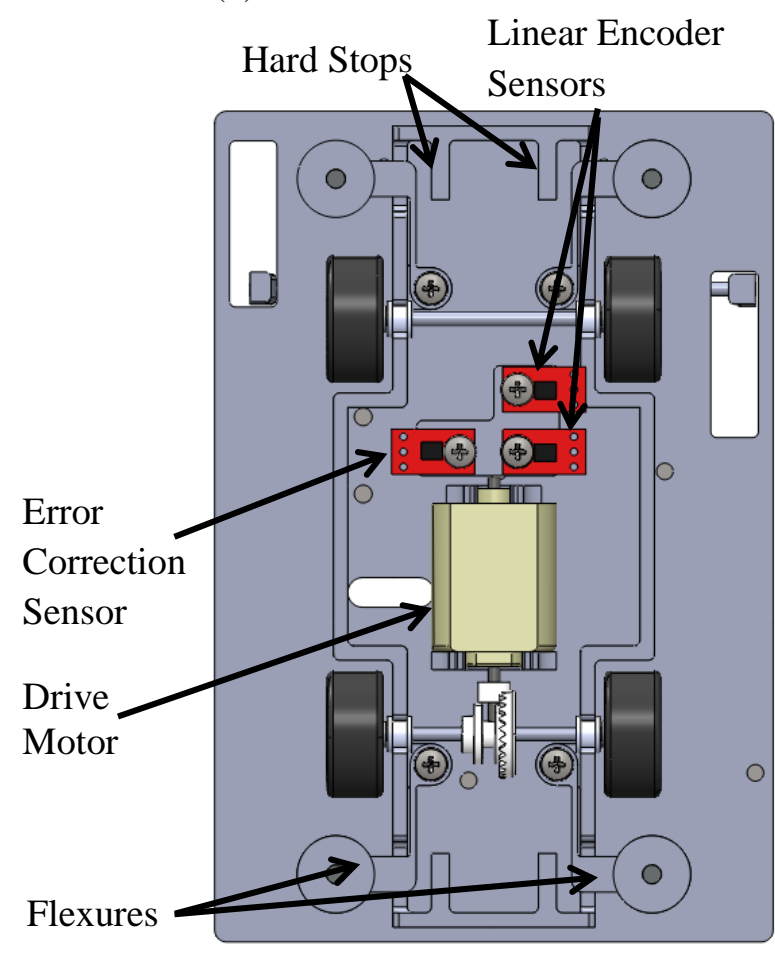

(b)

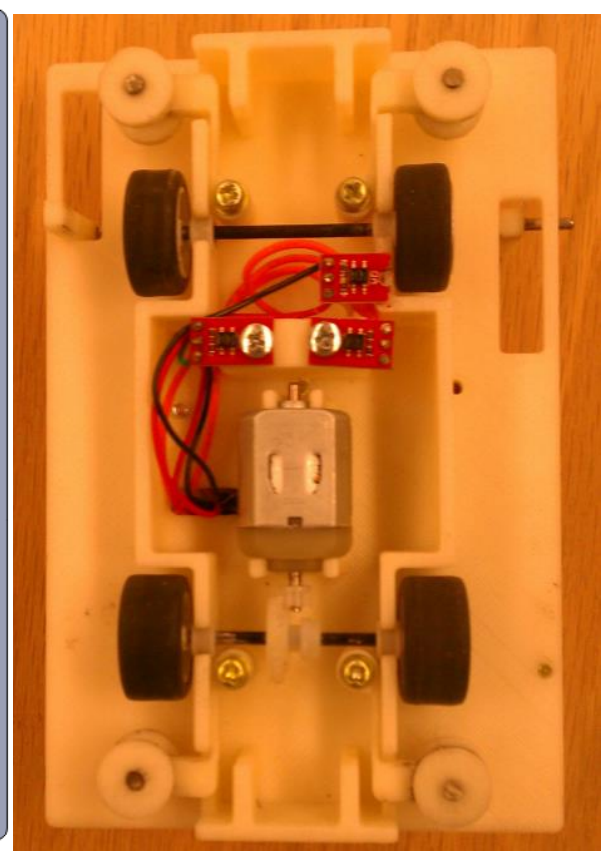

Figure 12. Bottom view of ATN vehicle. The drive mechanism of the vehicle is mounted to the bottom of the vehicle. Wheels mounted on flexures provide vehicle guidance within the track. Hard stops are behind each flexure to limit their deflection.

The sensors to control the vehicle are three QRE1113 infrared reflectance line sensors mounted on the bottom of the vehicle (Fairchield Semiconductor, 2009). The location of the sensors is shown in Figure 12. The line sensors count lines on a linear encoder mounted on the track. It does this by using the fact that a white surface has a different reflectivity from a black surface. To take advantage of this principle, the line sensor shines an infrared beam down and it reflects back onto a detector. The detector output is a different voltage based on the reflectivity of the material below it. This can be used to count lines on a linear encoder which allows the position of the vehicle to be known. 
The switching mechanism is operated by one 12 V DC motor from a Scalextric slot car kit. This motor drives a worm gear mechanism which then drives the switching mechanism up and down. Figure 13 (a) shows the switching mechanism in one position and Figure 13 (b) shows the switching mechanism in the other position. The worm gear was chosen to be self-locking so that the switch will stay in position even if a high force is imposed upon the switch from the track.
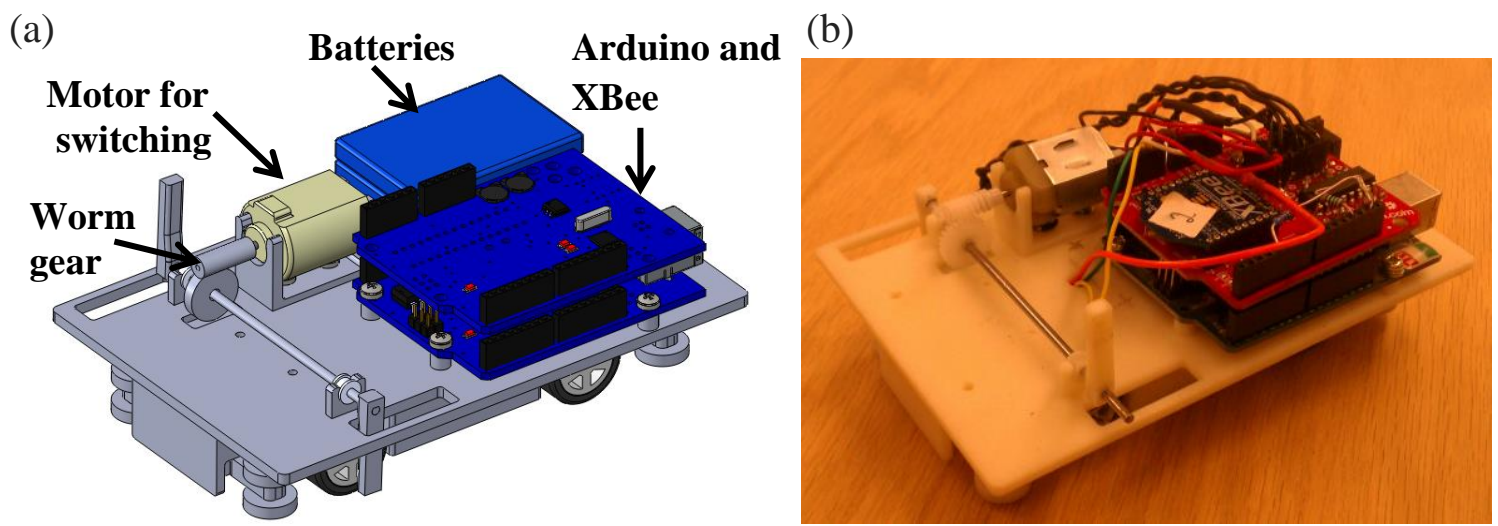

Figure 13. Isometric view of ATN vehicle. The vehicle switching mechanism is operated by the motor and worm gear mounted to the top of the vehicle. The batteries are directly behind the motor and the microcontroller is beside the batteries. (a) The rendered picture shows the left switch activated. (b) The actual prototype shows the right switch activated.

When the vehicle approaches a diverging track and the destination is to the left, the left switch is driven down to the outside of the track. The right switching mechanism is automatically driven to the up position as shown in Figure 13. This was an intentional safety feature implemented so that it was impossible for both switches to be engaged simultaneously on a diverging track. The left switching mechanism then slides on the outside of the left track, guiding the vehicle to the left. If the desired destination is to the 
right, the right switching mechanism is driven to the down position. An example of the vehicle moving right on a diverging section of track is shown in Figure 14.

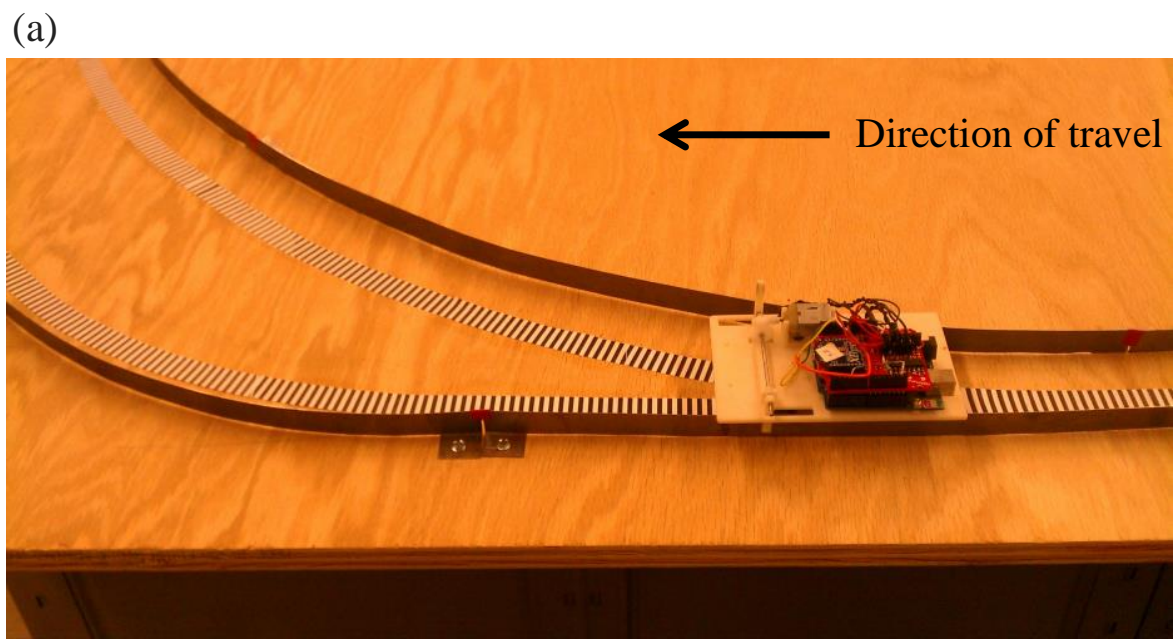

(b)

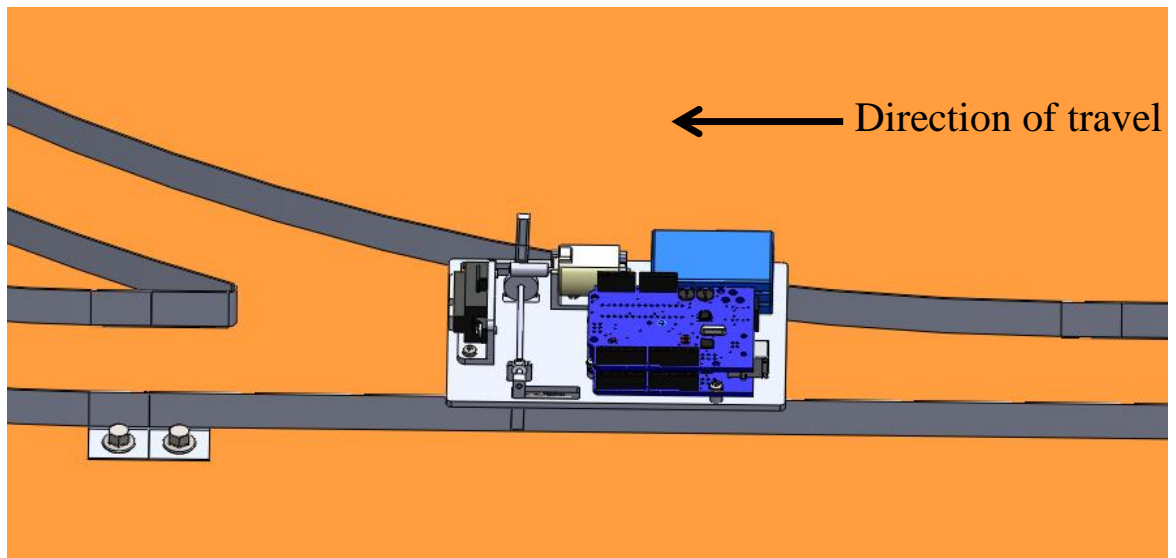

Figure 14. Isometric view of vehicle and track. The right side switching mechanism is engaged so that the vehicle will follow the outside track. (a) The prototype vehicle is moving along the track with the linear encoder. (b) The picture shows a rendering of the vehicle moving along the track.

Guide wheels were designed into the vehicle to guide the vehicle along the track and they were placed at the four corners of the vehicle. The wheel axles were mounted on flexures to allow compliance in the roller mechanism. This allows the rollers to take up error if the track is not perfectly constructed. The flexures can be seen at the four corners in Figure 12. 
The flexures were designed using the Solidworks finite element package. The desired deflection of each flexure was $3 \mathrm{~mm}$. This gave $6 \mathrm{~mm}$ of total tolerance in the width of the track. The finite element package was used to measure the stress on the flexure when loaded to achieve a $3 \mathrm{~mm}$ deflection. The length, width, and height of the flexure were varied to minimize the stress on the flexure. The results with $1 \mathrm{~N}$ of force applied are shown in Figure 15.

(a)

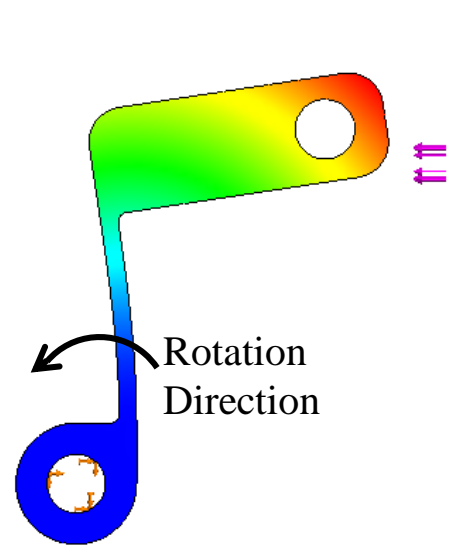

(b)

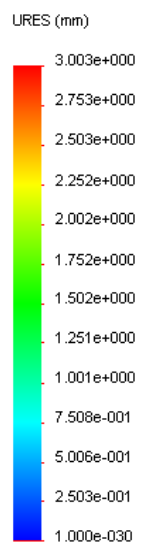

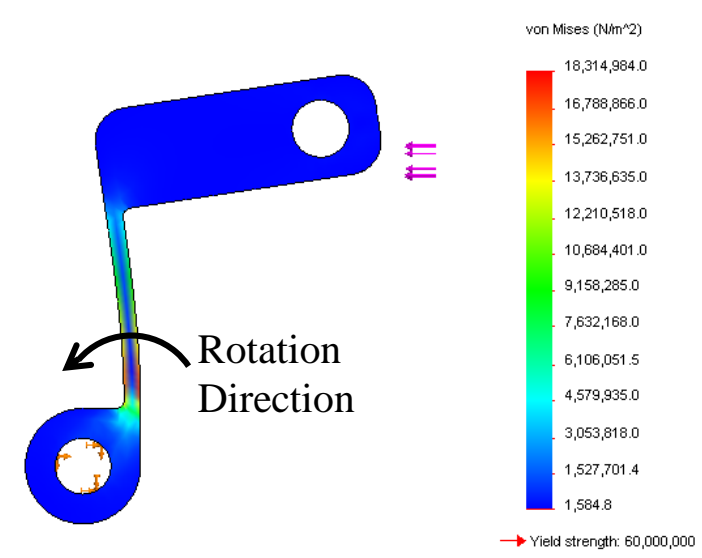

Figure 15. Finite element analysis of flexure. The flexure deflects $3 \mathrm{~mm}$ when a force of $1 \mathrm{~N}$ is applied, and at this deflection the von Mises stress is below the yield strength, so the flexure will not permanently deform.

The results show that the flexure deflects $3.003 \mathrm{~mm}$ and has a maximum von Mises stress of 18.31 MPa. This is more than three times the yield strength of the ABS plastic. Therefore, the flexure will have an infinite endurance limit and will not fail under fatigue. In order to ensure that the flexure was not damaged by overstressing the material, hard stops were incorporated into the design to prevent the flexure from deflecting more than $3 \mathrm{~mm}$. 
Linear encoder. A linear encoder was incorporated into the design so that the position and velocity of the vehicle could be determined at any location along the track. The linear encoder was designed to maximize the resolution of position detection while keeping in mind the hardware limits including microcontroller speed and the line sensor resolution. After testing many materials, it was found that white paper gave a low signal response while black ink on white paper gave a very high signal response. These materials maximized the range of the sensor. A section of the encoder design is shown in Figure 16.

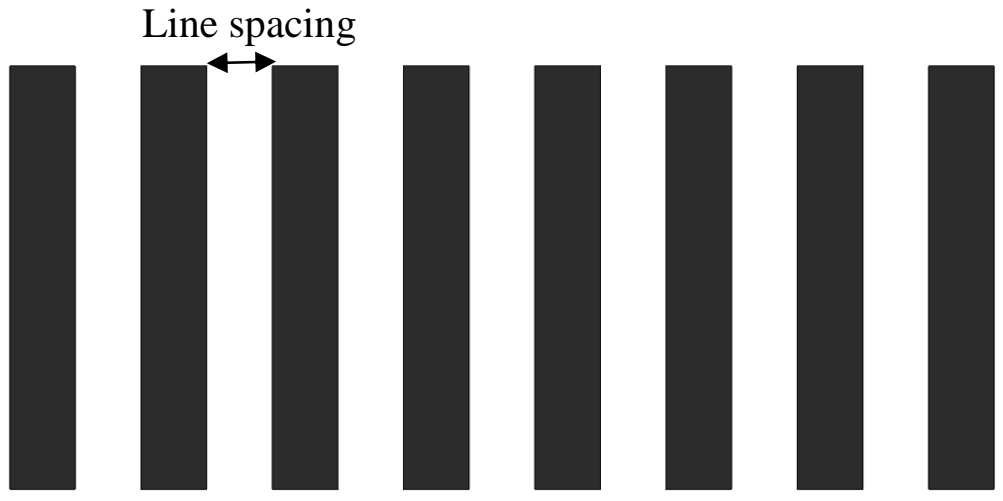

Figure 16. Section of linear encoder. The linear encoder design contains equally spaced white and black lines.

A test was conducted to determine the line spacing the line sensor could detect. Black rectangles that were $30 \mathrm{~mm}$ long were equally spaced on white paper to get equal amounts of white and black lines. The widths of the rectangles were varied from $2 \mathrm{~mm}$ to $6 \mathrm{~mm}$. The line sensor was then passed over the light and dark lines and the signal response was measured. The results are shown in Figure 17. 


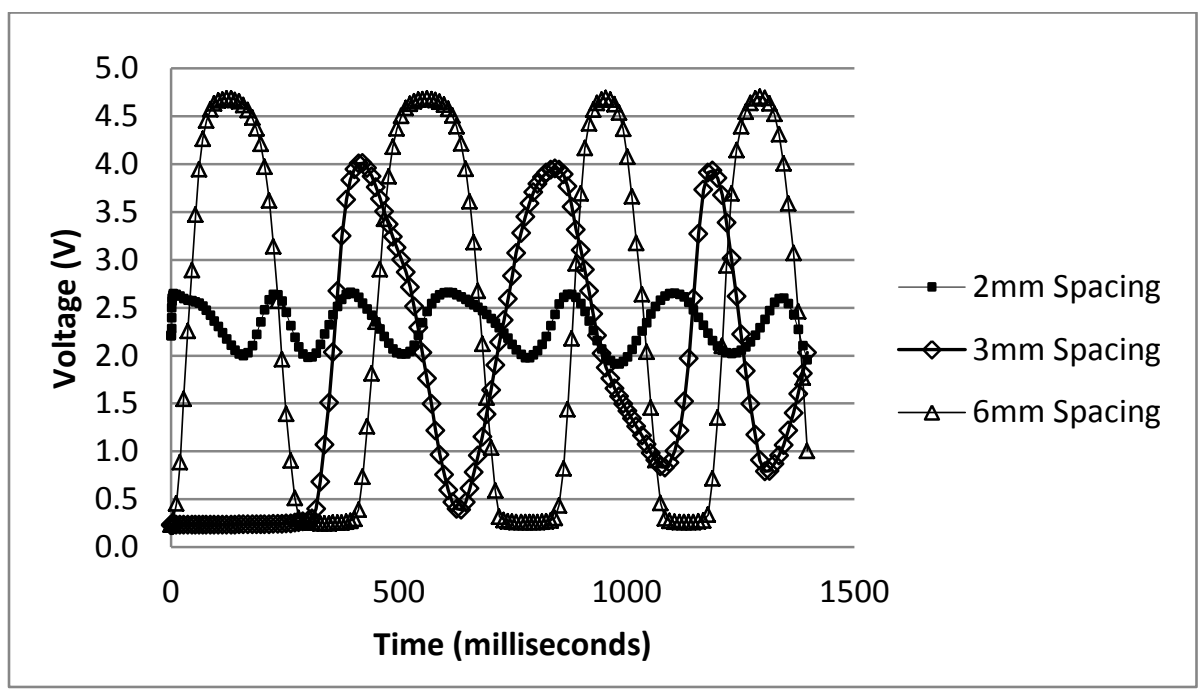

Figure 17. Line sensor signal response. The line sensor signal strength decreases as the spacing decreases because the line sensor averages the black and white lines together.

The data showed that the maximum signal difference between light and dark lines occurs when the spacing is $6 \mathrm{~mm}$. However, at $3 \mathrm{~mm}$ the signal difference was still greater than $3 \mathrm{~V}$. Once the spacing decreased to $2 \mathrm{~mm}$, there was a significant decrease in signal resolution. This made sense because the line sensor is $2.9 \mathrm{~mm}$ wide; therefore, if the resolution of the line encoder is below $2.9 \mathrm{~mm}$ the sensor would read an average of the two lines. A comparison of the linear encoder width and the infrared sensor width is shown in Figure 18. 


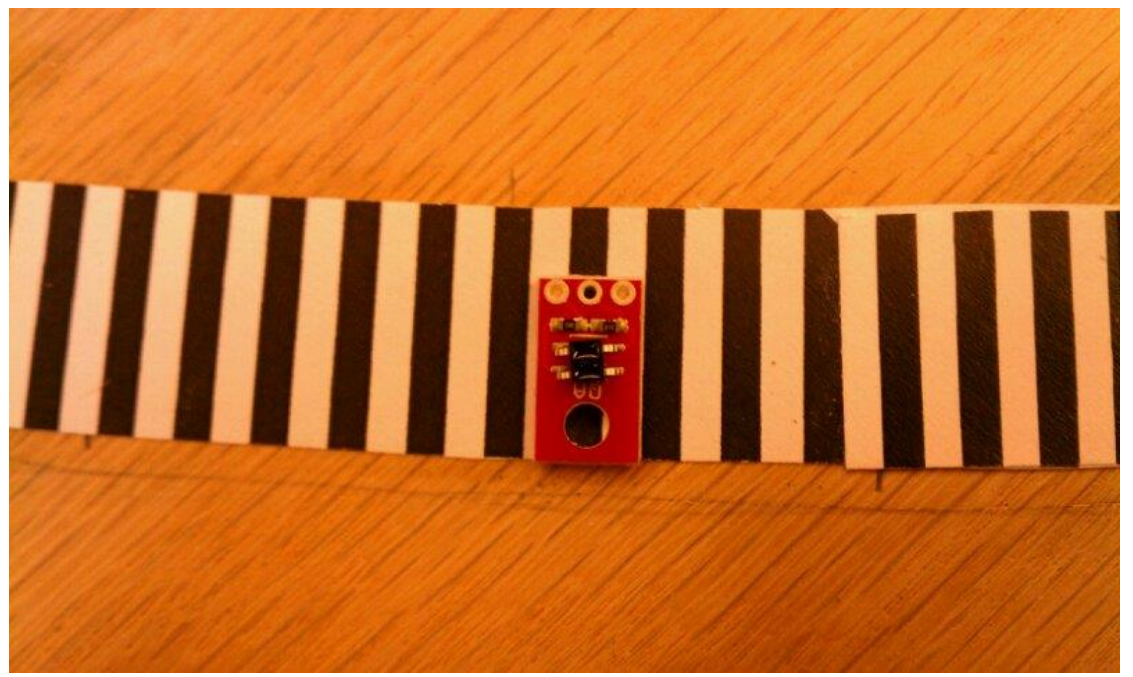

Figure 18. Linear encoder spacing. The picture shows that the black square QRE1113 sensor is just smaller in size than the $3 \mathrm{~mm}$ linear encoder.

The spacing of the line encoder was set to $3 \mathrm{~mm}$ to maximize resolution while maximizing the signal range of the line sensor.

Another important consideration with the linear encoder was to ensure that the microcontroller could sample the line sensor data fast enough when the vehicle was at maximum speed. The maximum speed of the vehicle was selected to be $800 \mathrm{~mm} / \mathrm{s}$ or 3.6 $\mathrm{km} / \mathrm{hr}$. This corresponds to a full-scale speed of 57 miles per hour since this is a $1 / 32$ scale prototype. This is more than adequate for most ATN systems because the national average travel speed is $14 \mathrm{mph}$ and heavy rail average travel speed is $20 \mathrm{mph}$ (Carnegie \& Voorhees, 2007). Additionally, the maximum speed of current commercially available ATN systems including Ultra, Vectus, and Cabintaxi is less than $40 \mathrm{mph}$ (Carnegie \& Voorhees, 2007).

If the vehicle is travelling at $800 \mathrm{~mm} / \mathrm{s}$ and the linear encoder has a spacing of 3 $\mathrm{mm}$, then the line sensor signal has a period of 7.5 milliseconds. The Nyquist sampling 
theorem states that the sampling rate of signal needs to be at minimum twice the maximum frequency of the signal. If the sampling frequency is less than this, the signal will be aliased. An aliased signal is a lower frequency signal than the original that produces the same set of sampled data. Therefore, with a $3 \mathrm{~mm}$ encoder and a vehicle travelling at maximum speed the sampling rate needed to have a maximum period of 3.75 milliseconds. The sampling rate was set to 1 millisecond to improve upon the integrity of the signal received. The microcontroller code is in Appendix G and the sampling rate was set on line 296.

Once the encoder was built on the track, it was tested to determine its robustness. In order to do this, code was written for the Arduino that counts a line every time the infrared line sensor voltage increased or decreased below a threshold corresponding to light and dark lines. After this code was written, the vehicle was deployed on the track. On average, at speeds varying from $100 \mathrm{~mm} / \mathrm{s}$ to $800 \mathrm{~mm} / \mathrm{s}$, the vehicle missed two lines every five laps. This corresponds to a position error of $6 \mathrm{~mm}$. In order to correct for this error, four rectangular black strips were placed at the four opposite sides of the track. An additional line sensor was placed on the bottom of the vehicle to read the four black strips. This is the sensor on the left in Figure 12. When the vehicle travels over a black square the vehicle location is reset to the location corresponding to the square. As a result, the location error will not accumulate.

The next step with the linear encoder was to determine the direction of travel of the vehicle. In order to decelerate quickly, the motor is driven in reverse, even while the vehicle is moving forward. Therefore, the voltage of the motor cannot be used to 
determine direction of travel. An additional line sensor was placed at the bottom of the vehicle to read the $3 \mathrm{~mm}$ encoder. The two sensors used to read the $3 \mathrm{~mm}$ encoder are shown in Figure 12. The sensors have a separation distance of $10.5 \mathrm{~mm}$; therefore, the voltage signals are 270 degrees out of phase with each other. The signal response of the two sensors while the vehicle is moving forward is shown in Figure 19.

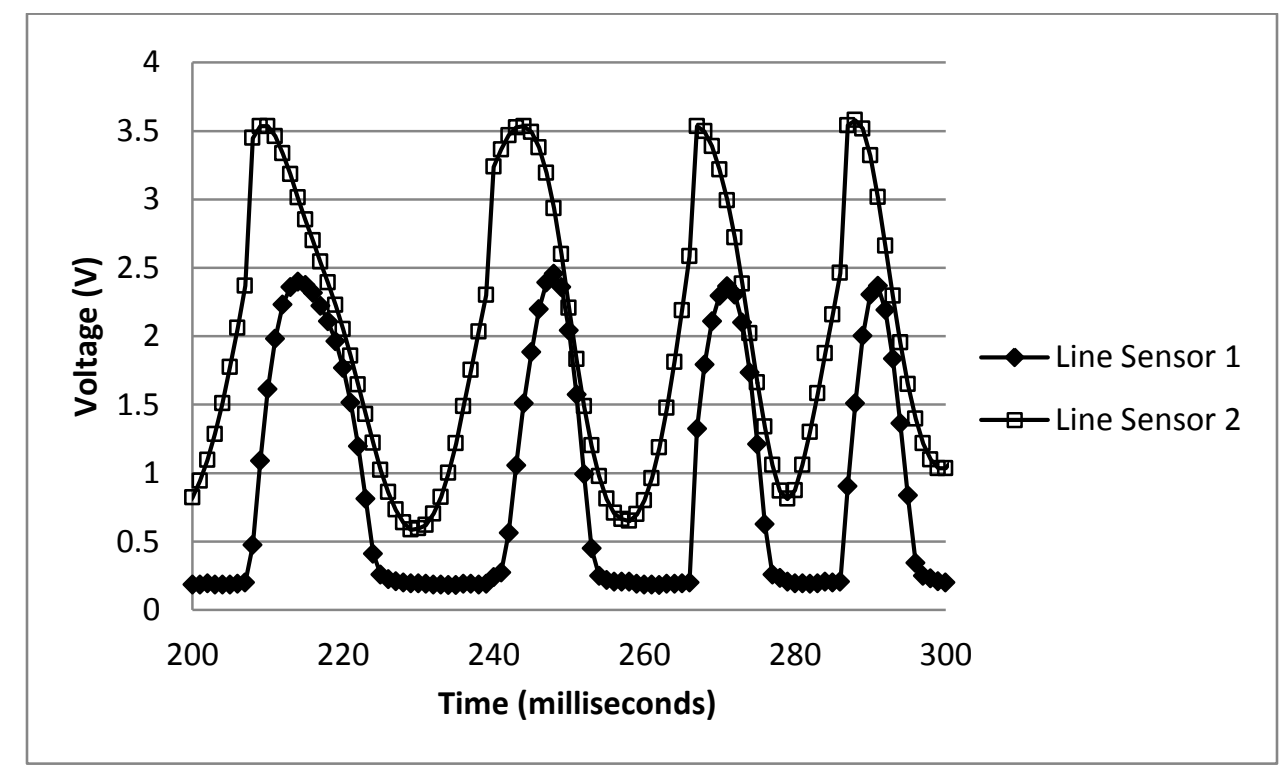

Figure 19. Quadrature encoder signal. Two line sensors are used to read the same set of encoder lines with a phase offset of 270 degrees. This allows the direction of travel to be determined.

The graph shows that the first line sensor signal peaks just after the second line sensor signal as the vehicle is moving forward. If the vehicle travels in reverse, then the first line sensor signal peaks just before the second line sensor. This information was used to write the microcontroller code on line 318 in Appendix G to determine direction based on if the first or second line sensor is leading.

Communication system. The communication system uses $2.4 \mathrm{GHz}$ XBee Series 2 wireless sensors to send information between the master controller and each vehicle 
(Digi, 2014). Each sensor is a transceiver so that it can send and receive information from each vehicle. XBee Series 2 sensors were selected over XBee Series 1 sensors because XBee Series 2 allows for mesh networking. This is beneficial for complex systems with many nodes because nodes will pass on information to other nodes when there is a lot of network traffic or there is a large distance between nodes.

Each vehicle has a XBee on board. It is connected to the Arduino microcontroller though a XBee shield that connects the RX and TX pins. This is shown in Figure 13. The Arduino sends out commands via the serial port to the XBee. The XBee then sends an RF signal out wirelessly, which other XBee nodes can pick up. The Arduino Serial port was set to a baud rate of 115,200 . This is the maximum data transfer rate to a XBee. This is important because the Arduino cannot perform any other tasks while sending data; therefore, it is important to send information quickly so that the microcontroller can perform other tasks.

Each XBee has a specific address, and information is sent to and from its address. Therefore, when sending data the address of the receiving node is specified. Additionally, once data is received, the address of the XBee that sent that data can be retrieved. In the scale system, the XBees on the vehicles report their location and velocity to the master controller every 50 milliseconds.

\section{System Identification}

The first step in the system identification process was to develop a model of the vehicle. In order to develop the vehicle model, a free body diagram was constructed for the vehicle. The free body diagram was constructed with the vehicle accelerating in the 
positive $\mathrm{x}$ direction; therefore, if the vehicle is decelerating or not accelerating at all, the equations are still valid by applying a negative or zero acceleration, respectively. The free body diagram of the chassis and axles are shown in Figure 20. In the following free body diagrams and equations, $\mathrm{F}$ is the symbol for force $(\mathrm{N})$ and $\mathrm{T}$ is the symbol for torque $(\mathrm{N} \cdot \mathrm{m})$.
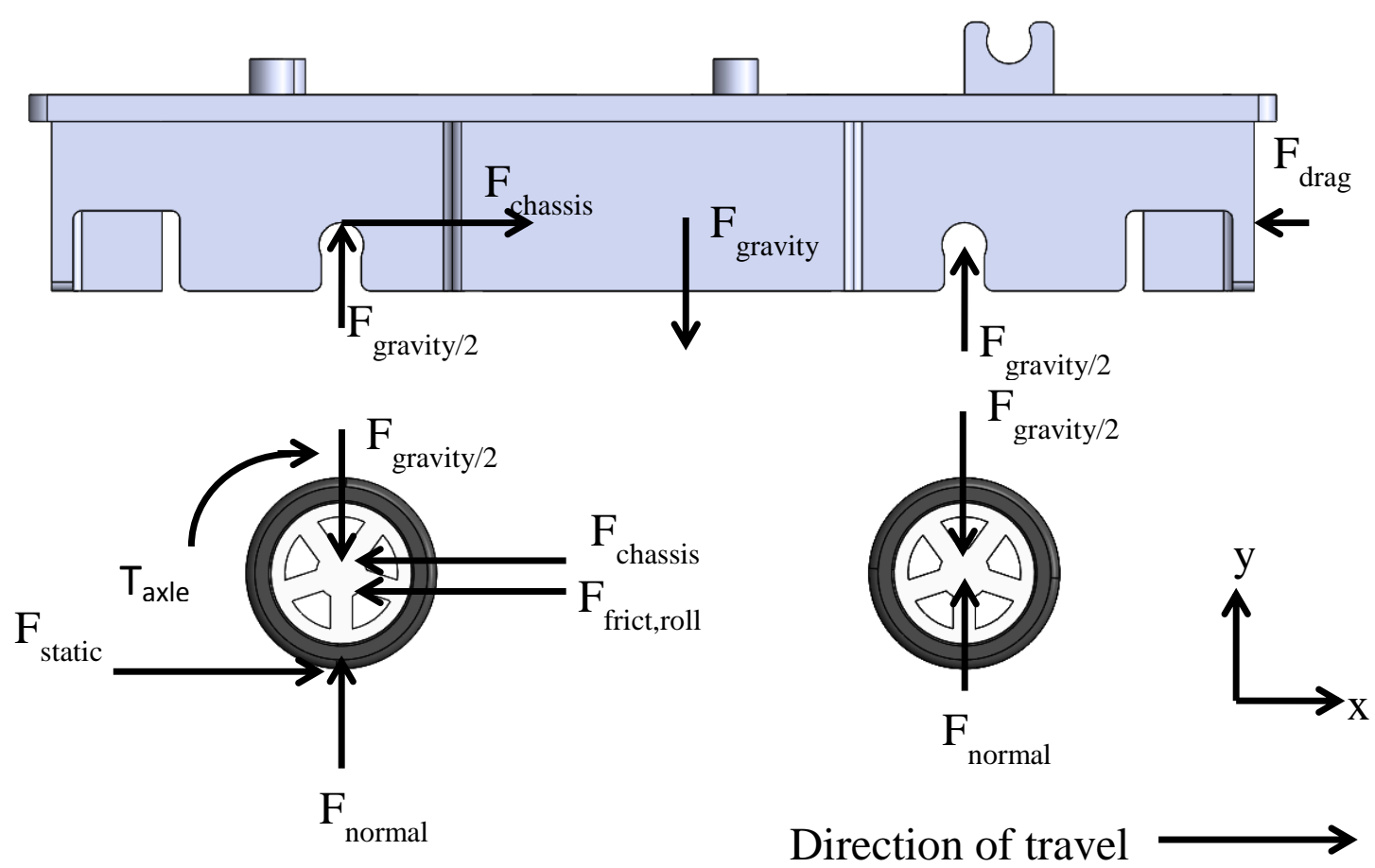

Figure 20. Free body diagram of vehicle. The free body diagram is shown for the vehicle accelerating in the positive $\mathrm{x}$ direction and $\mathrm{F}$ is used as the symbol for force.

Next, the free body diagram of the motor was developed, and it is shown in Figure 21. In this free body diagram, B is the coefficient of friction $(\mathrm{N} \cdot \mathrm{m} \cdot \mathrm{s} / \mathrm{rad})$ and $\omega$ is the angular velocity of the motor $(\mathrm{rad} / \mathrm{s})$. 


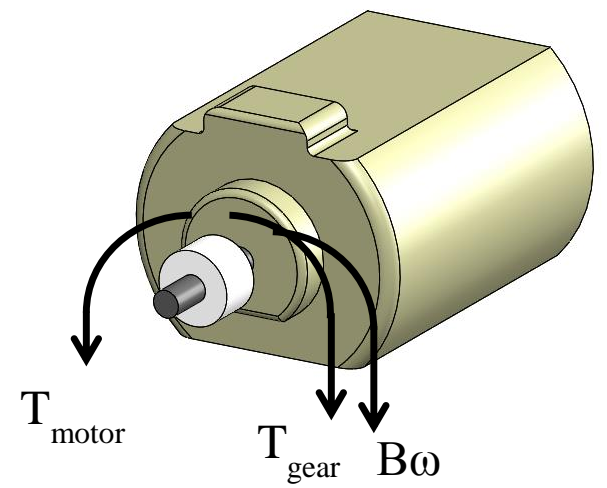

Figure 21. Free body diagram of the motor. The moments in the free body diagram are taken about the motor shaft axis where T is used for torque $(\mathrm{N} \cdot \mathrm{m}), \mathrm{B}$ is the friction coefficient $(\mathrm{N} \cdot \mathrm{m} \cdot \mathrm{s} / \mathrm{rad})$, and $\omega$ is the rotational velocity $(\mathrm{rad} / \mathrm{s})$.

Next, the electrical model of the motor was developed and it is shown in Figure

22.

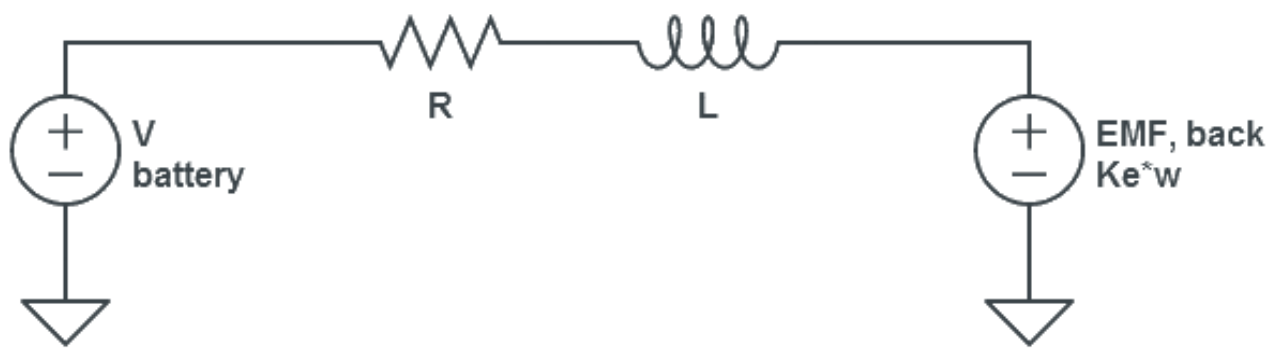

Figure 22. Electrical diagram of the motor. The simplified electrical model of the motor where $\mathrm{V}$ is input voltage $(\mathrm{V}), \mathrm{R}$ is resistance $(\Omega), \mathrm{L}$ is inductance $(\mathrm{H}), \mathrm{k}_{\mathrm{e}}$ is the motor velocity constant $(\mathrm{V} \cdot \mathrm{s})$, and $\omega$ is the rotational velocity of the motor $(\mathrm{rad} / \mathrm{s})$.

The most interesting direction in the free body diagram of the chassis is the $\mathrm{x}$ direction.

The equation of motion is written out below for this direction in equations 1 and 2 where $\mathrm{F}$ is force $(\mathrm{N}), \mathrm{m}$ is mass $(\mathrm{kg})$ and a is acceleration $\left(\mathrm{m} / \mathrm{s}^{2}\right)$.

$$
\begin{aligned}
& \sum F_{x}=m_{\text {chassis }} a \\
& m a=F_{\text {chassis }}-F_{\text {drag }}
\end{aligned}
$$


The vehicle drag can be calculated using equation 3 where $F_{d}$ is drag force $(N), C_{d}$ is the coefficient of drag (dimensionless), $\rho$ is the density of the fluid $\left(\mathrm{kg} / \mathrm{m}^{3}\right), \mathrm{v}$ is the relative velocity of the object the fluid $(\mathrm{m} / \mathrm{s})$, and $\mathrm{A}$ is the frontal area of the object $\left(\mathrm{m}^{2}\right)$ (The Engineering ToolBox, 2014).

$$
F_{d}=\frac{1}{2} C_{d} \rho v^{2} A
$$

The coefficient of drag for a flat plate is 1.98 (The Engineering ToolBox, 2014). This is the worst case scenario so it will give a conservative estimate for drag force. The drag force was then calculated using the properties of air and the dimensions of the vehicle.

$$
F_{d}=\frac{1}{2} * 1.98 * 1.18 \frac{\mathrm{kg}}{\mathrm{m}^{3}} *\left(800 \frac{\mathrm{m}}{\mathrm{s}}\right)^{2} * .0054 \mathrm{~m}^{2}=.07 \mathrm{~N}
$$

The drag force is near zero, and therefore, it was neglected, which left the equation 5.

$$
m_{\text {chassis }} a=F_{\text {chassis }}
$$

The equation of motion for the drive wheel in the $\mathrm{x}$ direction is shown in equations 6 and 7.

$$
\begin{aligned}
& \sum F_{x}=m_{\text {wheel }} a \\
& m_{\text {wheel }} a=F_{\text {static }}-F_{\text {chassis }}
\end{aligned}
$$

The value of $\mathrm{F}_{\text {chassis }}$ is known from equation 5.

$$
\begin{aligned}
& m_{\text {wheel }} a=F_{\text {static }}-m_{\text {chassis }} a \\
& m=m_{\text {total }}=m_{\text {wheel }}+m_{\text {chassis }} \\
& m a=F_{\text {static }}
\end{aligned}
$$


Next, the moment equation of motion was taken around the center of the rear drive wheel where $\mathrm{T}$ is torque $(\mathrm{N} \cdot \mathrm{m})$, I is moment of inertia $\left(\mathrm{kg} \cdot \mathrm{m}^{2}\right), \alpha$ is angular acceleration $\left(\mathrm{rad} / \mathrm{s}^{2}\right)$, and $\mathrm{r}$ is radius $(\mathrm{m})$.

$$
\begin{aligned}
& \sum T=I \alpha \\
& I \alpha=r_{\text {wheel }} F_{\text {static }}-T_{\text {axle }} \\
& I \alpha=r_{\text {wheel }} m a-T_{\text {axle }} \\
& r_{\text {wheel }} \alpha=-a \\
& I \alpha=-m r_{\text {wheel }}^{2} \alpha-T_{\text {axle }} \\
& \left(I+m r_{\text {wheel }}^{2}\right) \alpha=-T_{\text {axle }}
\end{aligned}
$$

The torque on the axle and the torque on the motor are related by the gear ratio between the gear on the motor and the gear on the shaft. The gears have a 3:1 gear ratio and it reverses the direction of motion. The moment equation of motion around the motor shaft is derived starting with equation 17 where $\theta$ is angular position (rad).

$$
\begin{aligned}
& \sum T=I \alpha \\
& I_{\text {motor }} \alpha_{\text {motor }}=T_{\text {motor }}-T_{\text {gear }}-B \omega \\
& I_{\text {motor }} \alpha_{\text {motor }}=T_{\text {motor }}+\frac{T_{\text {axle }}}{3}-B \omega \\
& I_{\text {motor }} \alpha_{\text {motor }}=T_{\text {motor }}-\frac{\left(I_{\text {wheel }}+m r_{\text {wheel }}^{2}\right) \alpha_{\text {wheel }}}{3}-B \omega \\
& \alpha_{\text {motor }}=3 * \alpha_{\text {wheel }} \\
& I_{\text {motor }} \alpha_{\text {motor }}=T_{\text {motor }}-\frac{\left(I_{\text {wheel }}+m r_{\text {wheel }}^{2}\right) \alpha_{\text {wheel }}}{3}-B \omega \\
& I_{\text {motor }} \alpha_{\text {motor }}=T_{\text {motor }}-\frac{\left(I_{\text {wheel }}+m r_{\text {wheel }}^{2}\right) \alpha_{\text {motor }}}{9}-B \omega
\end{aligned}
$$




$$
\begin{aligned}
& T_{\text {motor }}=\left(I_{\text {motor }}+\frac{I_{\text {wheel }}+m r_{\text {wheel }}^{2}}{9}\right) \alpha_{\text {motor }}+B \omega \\
& T_{\text {motor }}=\left(I_{\text {motor }}+\frac{I_{\text {wheel }}+m r_{\text {wheel }}^{2}}{9}\right) \ddot{\theta}+B \dot{\theta}
\end{aligned}
$$

The electrical model of the motor is described with the following equations where $\mathrm{V}$ is voltage $(V), R$ is resistance $(\Omega)$, I is current $(A), L$ is inductance $(H), k_{e}$ is the motor velocity constant $(\mathrm{N} \cdot \mathrm{m} \cdot \mathrm{s} / \mathrm{rad}), \omega$ is the angular velocity $(\mathrm{rad} / \mathrm{s})$, and $\theta$ is angular position (rad).

$$
\begin{aligned}
& V-R I-L \frac{d I}{d t}-V_{e m f, b a c k}=0 \\
& V_{e m f, b a c k}=k_{e} \omega \\
& V-R I-L \frac{d I}{d t}-k_{e} \dot{\theta}=0
\end{aligned}
$$

The motor torque is related to the current by the following equation where $\mathrm{k}_{\mathrm{i}}$ is the motor size constant $(\mathrm{N} \cdot \mathrm{m} / \mathrm{A})$.

$$
T_{\text {motor }}=k_{i} I
$$

The torque constant $\mathrm{k}_{\mathrm{i}}$ is equal to the voltage constant $\mathrm{k}_{\mathrm{e}}$ when using International System of Units (SI) so the constant $\mathrm{k}$ will be used to represent both numeric values in equations that follow. Using Laplace transforms, the transfer function of the electrical equations were combined with the mechanical equations of motion to determine the overall system equation.

$$
\begin{aligned}
& k I=\left(I_{\text {motor }}+\frac{I_{\text {wheel }}+m r_{\text {wheel }}^{2}}{9}\right) \ddot{\theta}+B \dot{\theta} \\
& I=\frac{9 I_{\text {motor }}+I_{\text {wheel }}+m r_{\text {wheel }}^{2}}{9 k} \ddot{\theta}+\frac{B}{k} \dot{\theta} \\
& I(s)=\frac{9 I_{\text {motor }}+I_{\text {wheel }}+m r_{\text {wheel }}^{2}}{9 k} s^{2} \theta(s)+\frac{B}{k} s \theta(s)
\end{aligned}
$$




$$
\frac{I(s)}{\theta(s)}=\frac{\left(9 I_{m o t o r}+I_{w h e e l}+m r_{w h e e l}^{2}\right) s^{2}+9 B s}{9 k}
$$

Next, the transfer function between the motor shaft angle and the velocity was derived.

$$
\begin{aligned}
& V(s)-R I(s)-L s I(s)-\mathrm{ks} \theta(s)=0 \\
& V(s)-(R+s L) \frac{\left(9 I_{\text {motor }}+I_{\text {wheel }}+m r_{\text {wheel }}^{2}\right) s^{2}+9 B s}{9 k} \theta(s)-k s \theta(s)=0 \\
& \frac{V(s)}{\theta(s)}=(R+s L) \frac{\left(9 I_{\text {motor }}+I_{\text {wheel }}+m r_{\text {wheel }}^{2}\right) s^{2}+9 B s}{9 k}+k s \\
& \frac{V(s)}{\theta(s)}=\frac{(R+s L)\left[\left(9 I_{\text {motor }}+I_{\text {wheel }}+m r_{\text {wheel }}^{2}\right) s^{2}+9 B s\right]}{9 k}+\frac{9 k^{2} s}{9 k} \\
& \frac{\theta(s)}{V(s)}=\frac{9 k}{(R+s L)\left[\left(9 I_{\text {motor }}+I_{\text {wheel }}+m r_{\text {wheel }}^{2}\right) s^{2}+9 B s\right]+9 k^{2} s} \\
& \frac{\theta(s)}{V(s)}=\frac{9 k}{s\left[(R+s L)\left[\left(9 I_{\text {motor }}+I_{\text {wheel }}+m r_{\text {wheel }}^{2}\right) s+9 B+9 k^{2}\right]\right.}
\end{aligned}
$$

Equation 39 gives the position of the rotor when a given voltage is applied.

Equation 39 can be greatly simplified because the moment of inertia of the wheels and motor is negligible compared to the mass of the vehicle. Additionally, the inductance of the motor is negligible. This is shown later when the motor parameters are determined. The simplified equation is shown in equation 40.

$$
\frac{\theta(s)}{V(s)}=\frac{9 k}{s\left[R m r_{\text {wheel }}^{2} s+9 B+9 k^{2}\right]}
$$

The equivalent transfer function for velocity of the vehicle is shown in equation 41 .

$$
\frac{\Omega(s)}{V(s)}=\frac{9 k}{R m r_{w h e e l}^{2} s+9 B+9 k^{2}}
$$

The next step in the system identification process was to characterize the Mabuchi C8146 motor used in the system. The motor constants were determined by running a series of tests. 
First, the motor resistance was determined by applying a voltage to the motor while stalling the rotor and measuring the current that the motor produced. The measurement was taken after five seconds to ensure that the voltage drop due to the inductance of the motor was zero. This was then repeated for several voltages and several positions of the armature to ensure that there were no variations due to the locations of the brushes within the motor. The resistance of the motor was found by dividing the voltage by the current, and then all resistances were averaged to get a value of $7.74 \mathrm{ohms}$. This method applies because the voltage drop due to the inductor is $0 \mathrm{~V}$ since the current is not changing and the back electromotive force is $0 \mathrm{~V}$ since the rotor is not rotating.

Next an inductance capacitance resistance (LCR) meter was used to measure the inductance of the motor. The meter had an average reading of $1.5 \mathrm{mH}$. This value of inductance is small. This is typical for a small DC motor. The electrical time constant of the motor was calculated using the following equation where R is resistance $(\Omega)$ and $\mathrm{L}$ is inductance (H) (Movellan, 2013).

$$
\tau_{\mathrm{e}}=\frac{\mathrm{R}}{\mathrm{L}}
$$

The electrical time constant is $1.94 \times 10^{-4} \mathrm{~s}$. It can be considered negligible in the system transfer function because it is significantly smaller than the mechanical time constant.

The motor voltage constant was determined by applying varying voltages to the motor and measuring the resulting speed with no load on the motor shaft. This voltage was changed in $0.25 \mathrm{~V}$ increments from $0 \mathrm{~V}$ to $12 \mathrm{~V}$ and measurements were taken at each step. The angular velocity was measured using a Cole Parmer Model 8211 Digital 
Phototach. Using the electrical model of the motor shown above, the inductance can be eliminated because the current is operating at steady state when the measurement is made. Therefore, the equation 28 reduces to equation 43 .

$$
V-R I-k \omega=0
$$

At this point everything is known except the voltage constant so the voltage constant can be determined from equation 44 .

$$
V-R I=k \omega
$$

Figure 23 shows equation 44 in graphical form. The slope of the graph is the voltage constant. The value of $\mathrm{k}_{\mathrm{e}}$ is 0.005 Volt*seconds.

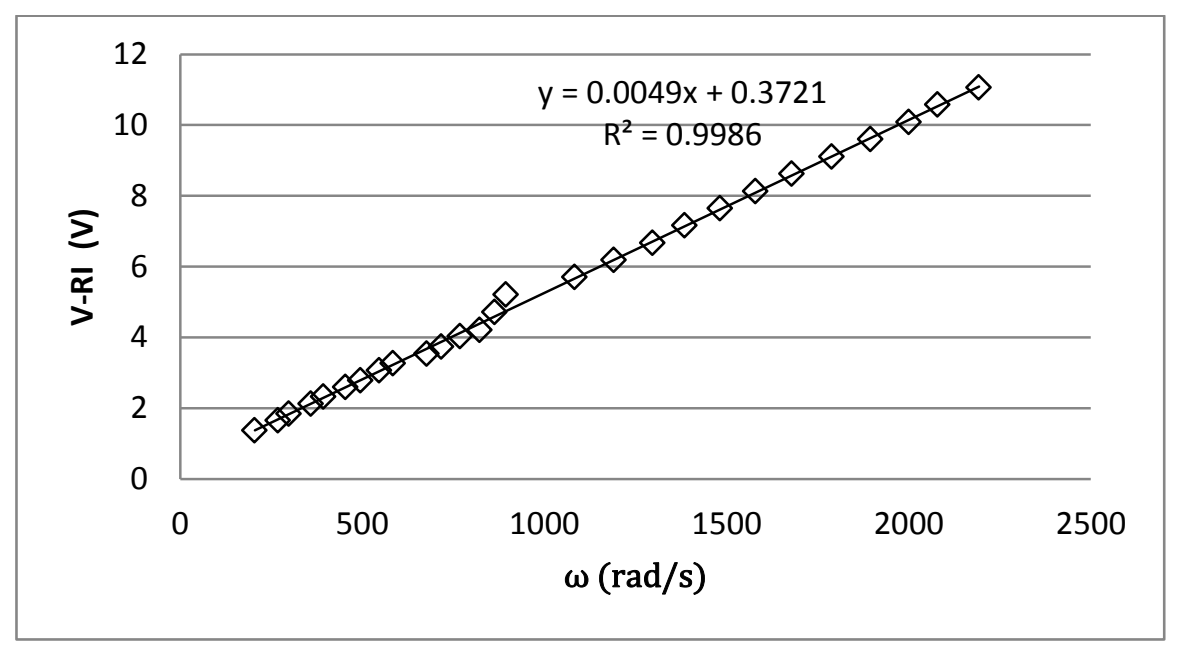

Figure 23. Motor characterization of $\mathrm{k}_{\mathrm{e}}$. The slope of the plotted data is the voltage constant of the motor.

The next step was to determine the vehicle's physical parameters. The vehicle's mass is 260.5 grams and the wheel radius is $11.5 \mathrm{~mm}$.

A 2.7 V step was applied to the vehicle and the speed response was measured using the $3 \mathrm{~mm}$ linear encoder. The response was then imported into Matlab 2013b and the software was used to curve fit the response. The results are shown in Figure 24. 


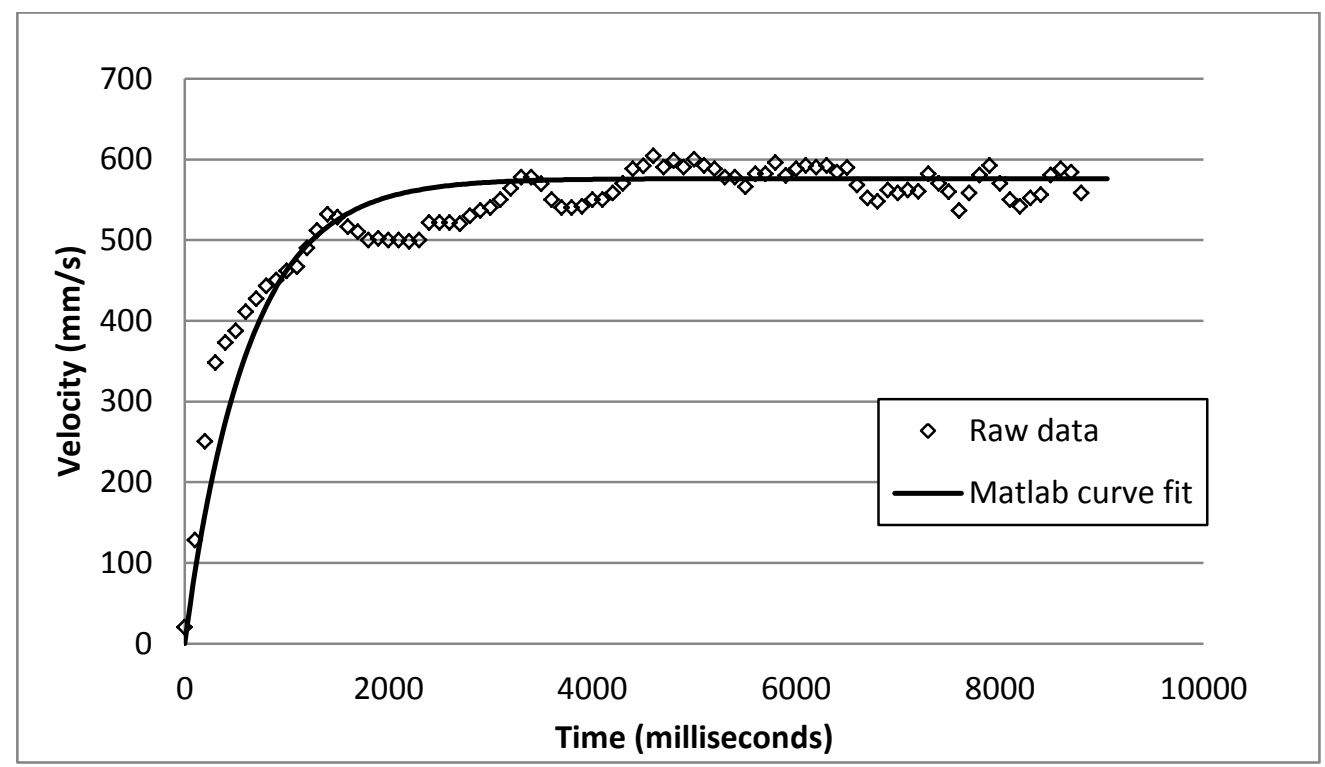

Figure 24. System plant identification. This graph compares experimental data with a transfer function model generated in Matlab.

The function determining the plant transfer function is shown in equation 45 .

$$
\frac{\Omega(s)}{V(s)}=\frac{18.56}{0.6186 s+1}
$$

The corresponding transfer function with position as the output is shown in equation 46 .

$$
\frac{\theta(s)}{V(s)}=\frac{18.56}{0.6186 s^{2}+s}
$$

The transfer function in equation 46 was used to determine the appropriate controller for the system.

The time constant for the first order electromechanical system is $0.6186 \mathrm{~s}$. This is significantly greater than the electrical time constant of $1.94 \times 10^{-4} \mathrm{~s}$ calculated earlier. Therefore, the mechanical dynamics of the system will dominate the system response which means that the first order mechanical approximation of the system is valid. 


\section{System Controller}

The transfer function derived in the previous section was used to determine the response of the system with unity feedback and a proportional gain. The block diagram of the system is shown in Figure 25.

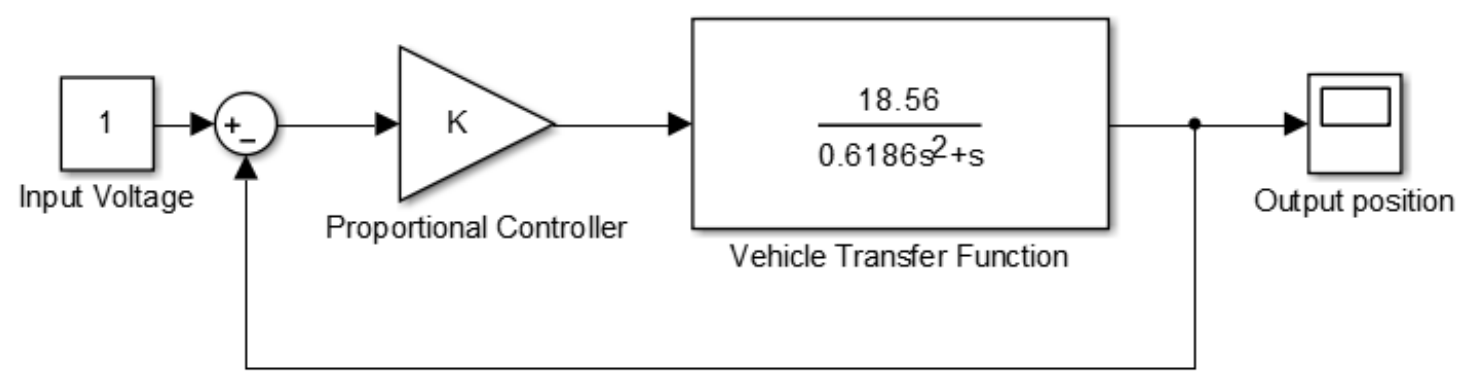

Figure 25. Block diagram of a system with a proportional gain controller. The PID controller consists of only a simple proportional gain.

The corresponding root locus of the system is shown in Figure 26. The root locus shown in Figure 26 and the unit step response of the system shown in Figure 27 were generated using Matlab. The code to generate this data is shown in Appendix E. 


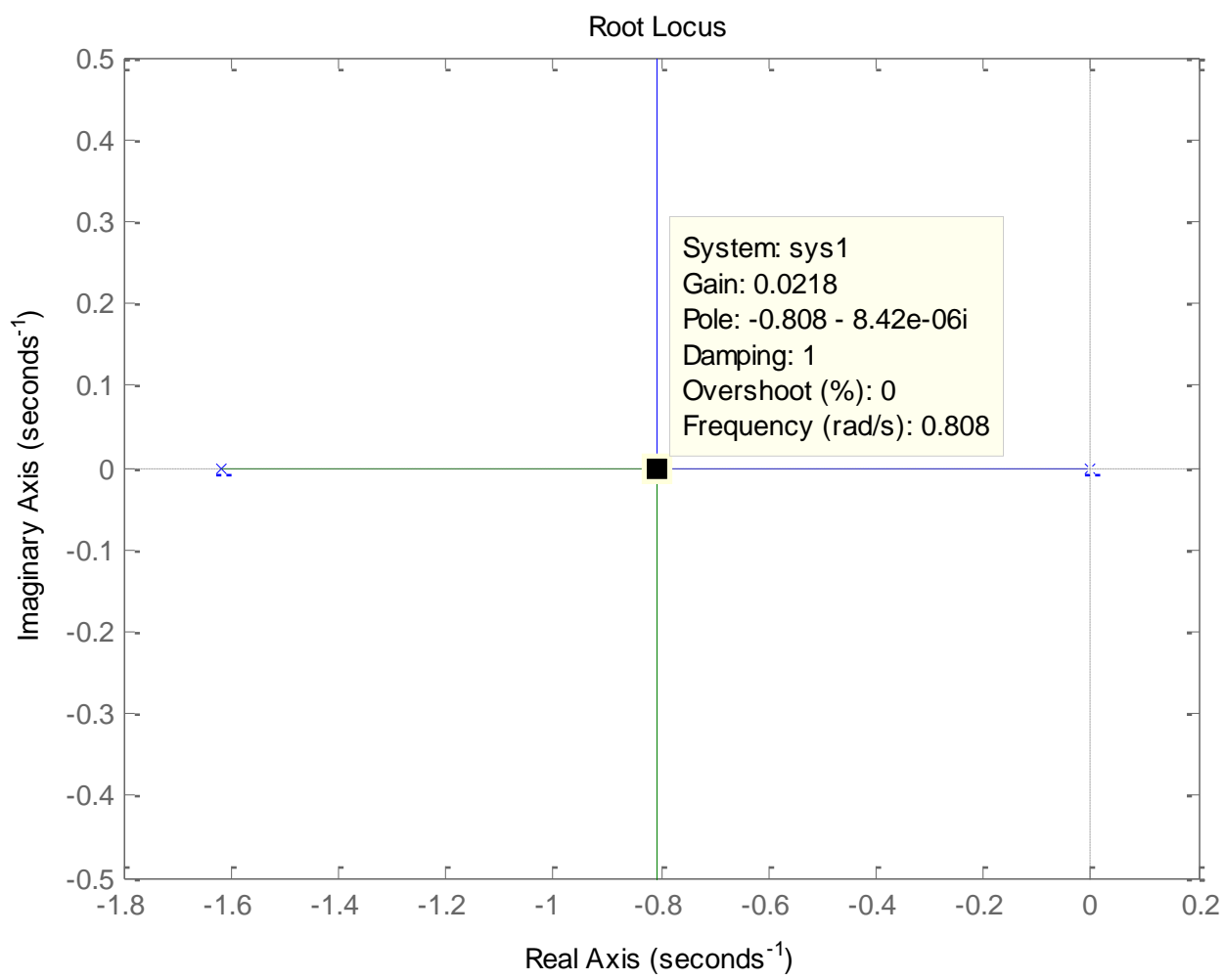

Figure 26. Root locus of the open loop transfer function. The fastest response time of the closed loop system with a proportional gain has a proportional gain equal to 0.0218 .

The system open loop transfer function is simply the vehicle transfer function since there is only a proportional controller. Based on the root locus, the rise time that can be achieved is 4.15 seconds at a gain of 0.0218 if there is no overshoot. This is determined by finding the maximum gain where the poles are still on the real axis. Overshoot is not desired because in a real life system it would be undesirable to overshoot the platform when pulling into a station. The system response at a gain of 0.0218 is shown in Figure 27. 


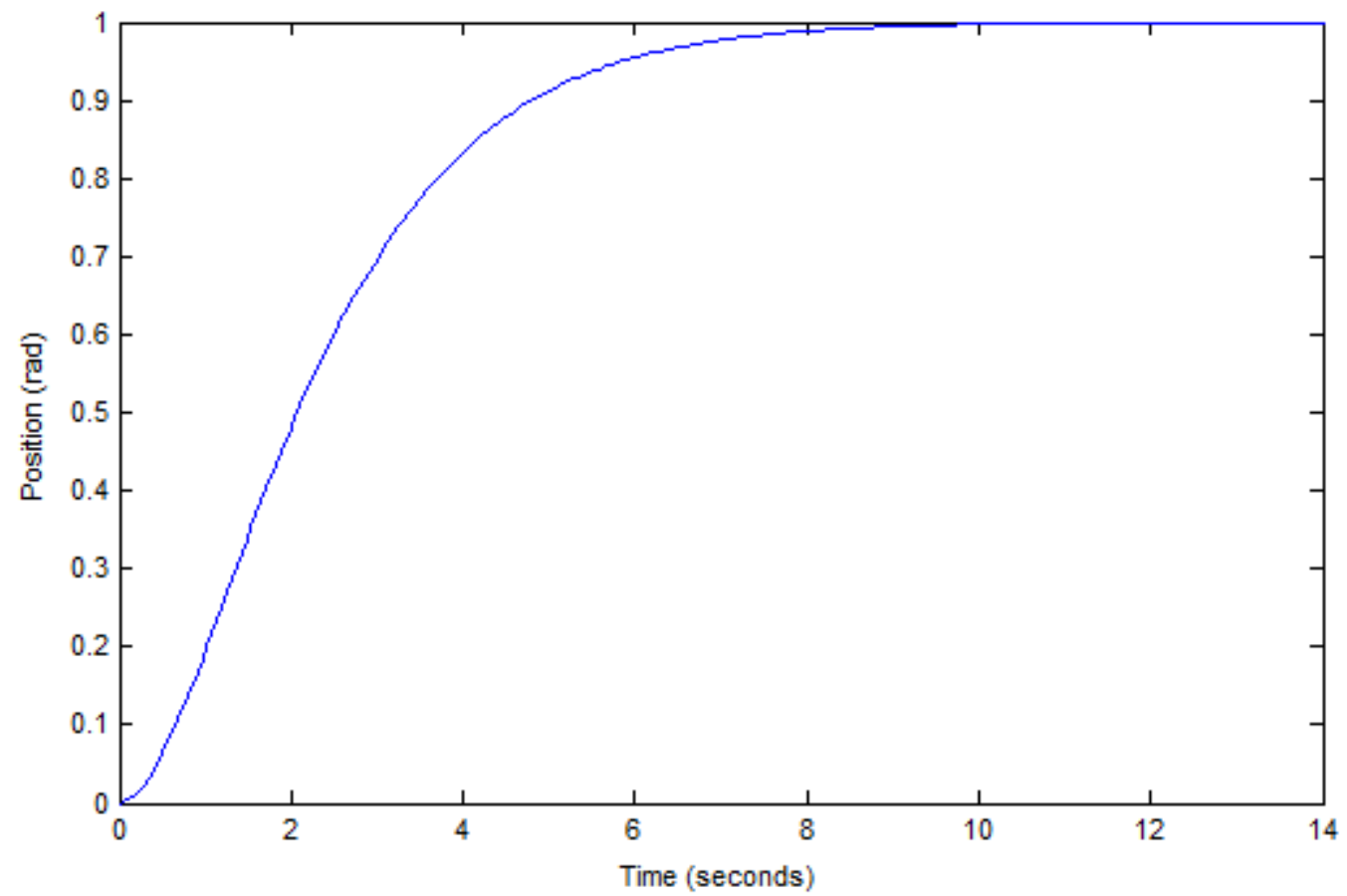

Figure 27. Step response of the vehicle with a proportional gain controller. The step response of the best possible rise time with no overshoot has a gain equal to 0.0218 .

Position controller. It was desired that the steady state error in the system be driven to zero. This is the case with position control since there is an integrator in the denominator of the plant transfer function. This is shown in equation 46. However, it was still desirable to achieve improved response times with the system while adjusting the integral control to overcome friction in the system. Therefore, a proportional integral derivative (PID) controller was developed to improve the response times. The block diagram of the controller and overall system is shown in Figure 28. 


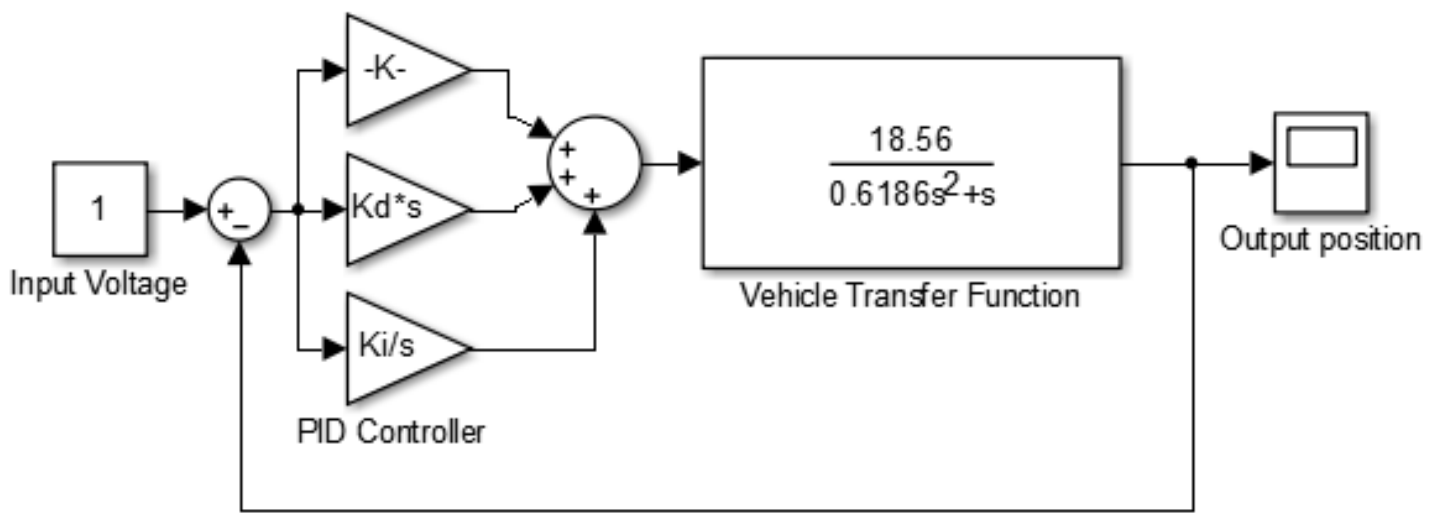

Figure 28. Block diagram of the system with a PID controller. The PID controller was implemented to improve rise time and minimize steady state error.

To initially tune the parameters in the controller the Ziegler-Nichols (1993) method was used. In this method, the integral and derivative terms in the controller are initially set to zero. Then the proportional gain $(\mathrm{Kp})$ is incrementally increased until the system undergoes sustained oscillations when a step response is input into the system. Sustained oscillations occur when the proportional gain is set too high and the system response does not decay to a steady state value.

The system response to a step voltage input was measured by loading the proportional gain on the microcontroller, setting the vehicle up to wirelessly transmit position and time values to the master controller, and then applying the step voltage change to the vehicle. The proportional gain was increased on the vehicle control system incrementally until is underwent sustained oscillations. The recorded response is shown in Figure 29 for an ultimate proportional gain $(\mathrm{Ku})$ of 3. 


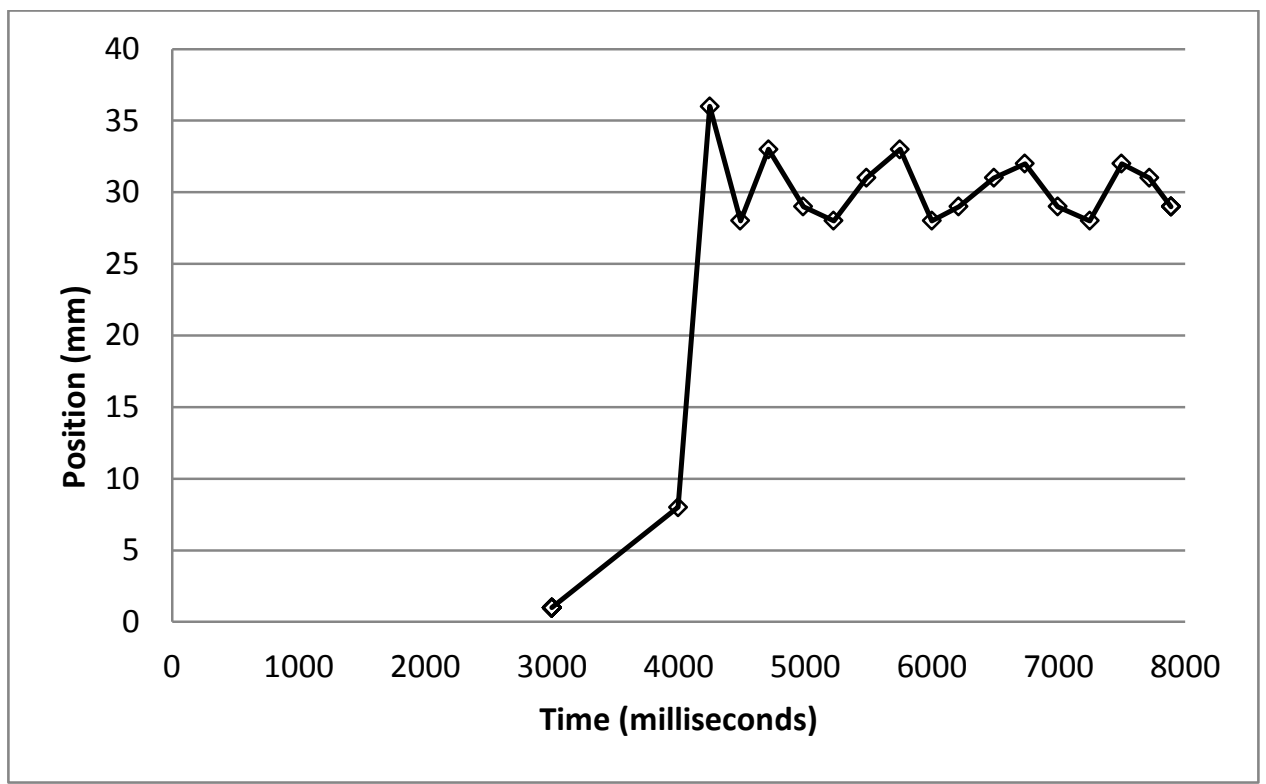

Figure 29. Zeigler-Nichols criterion data for position control. The proportional gain of the system was increased until the system underwent sustained oscillations. The period of oscillation was used to determine the gains of the PID controller.

The oscillation period $\left(\mathrm{T}_{\mathrm{u}}\right)$ was 955 milliseconds, and the Ziegler-Nichols

criterion was used to determine appropriate gains for the system. For a PI controller, the gains are set using equations 47 and 48 (Ziegler \& Nichols, 1993).

$$
\begin{aligned}
& K_{p}=0.45 K_{u}=1.35 \\
& \mathrm{~K}_{\mathrm{i}}=\frac{1.2 \mathrm{~K}_{\mathrm{p}}}{\mathrm{T}_{\mathrm{u}}}=1.7
\end{aligned}
$$

The response of the system using the Zeigler-Nichols parameters was stable with an improved response time; however, it did undergo some overshoot, which is typical of Zeigler-Nichols tuning. Therefore, the system parameters were tweaked using the Zeigler-Nichols parameters as a starting point. The goal was to eliminate the steady state error due to friction while increasing the response time. The final parameters used in the 
system were $\mathrm{K}_{\mathrm{p}}=1.5$ and $\mathrm{K}_{\mathrm{i}}=0.03$. The system response with these parameters is shown in Figure 30.

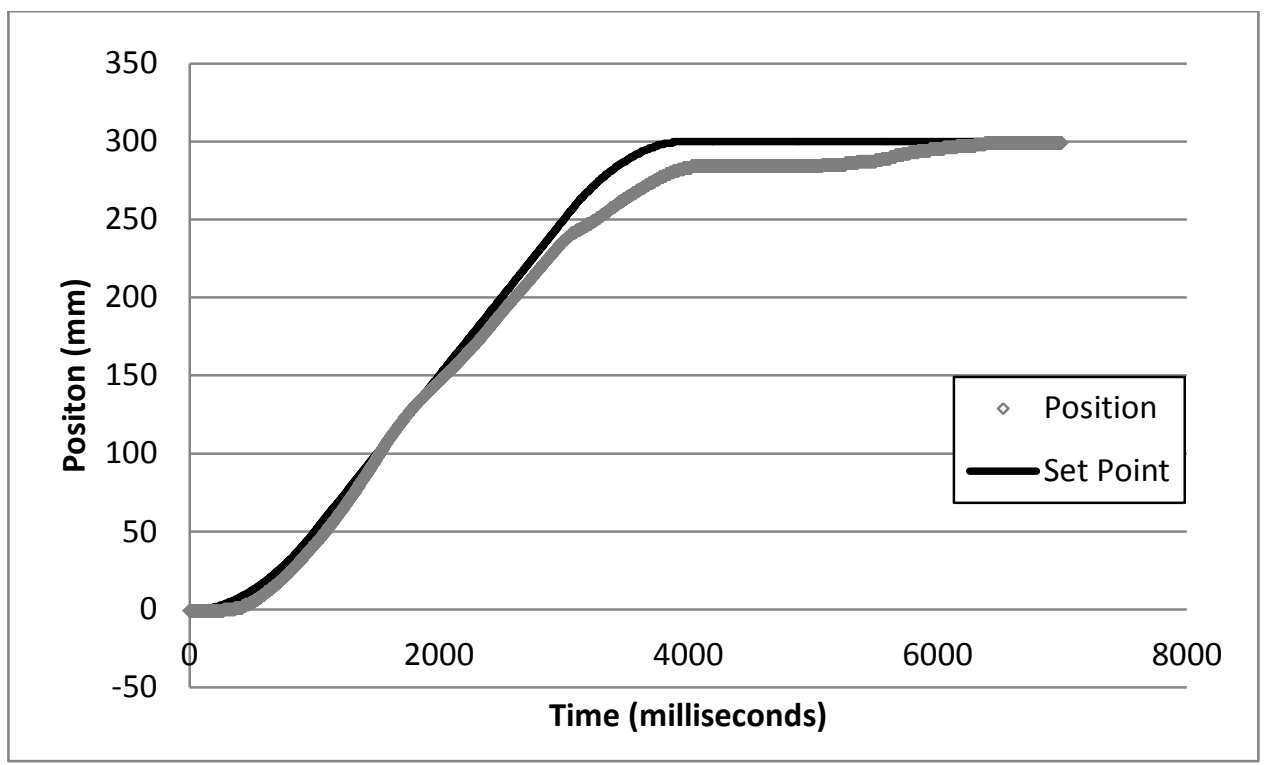

Figure 30. Position with PID controller. The vehicle position is compared to the position set point.

Figure 30 shows a move from zero to $300 \mathrm{~mm}$ with a constantly ramping set point. The response time of the system is 2.91 seconds. The position follows the set point well, but at the end of the move the position falls away from the set point. This is because of increased friction that occurred when the vehicle entered a corner. However, the integral term increased and moved the vehicle to the appropriate set point eliminating the steady state error.

Cascaded position velocity controller. The next step in the system was to develop a velocity controller to control the speed of the vehicle while it is travelling around the track. This is necessary to allow greater position control and to help ensure velocity stability when there is a position error. In Figure 31, the velocity of the vehicle 
was measured when a constant input voltage was applied to the dc motor controlling the vehicle.

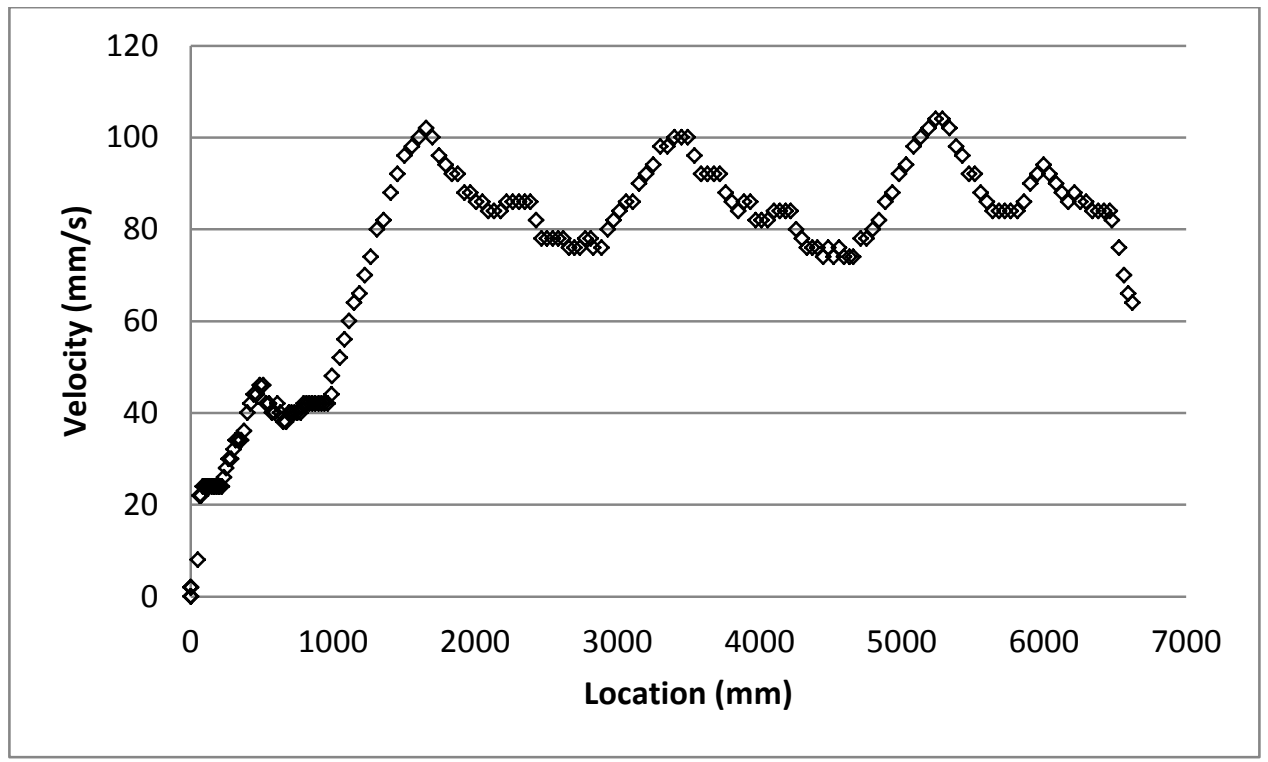

Figure 31 . Velocity with a step voltage input. The graph shows the velocity of the vehicle as it travels around the track once.

Figure 31 shows that the velocity of the vehicle is highly dependent on the position of the vehicle on the track. The dips in velocity occur as the vehicle enters corners on the track where there is a higher friction. A cascaded position velocity controller is needed to allow the vehicle to travel at a constant speed when it is travelling around the track. A cascaded position velocity controller does this by forcing the position and the velocity to converge to predefined set points for position and velocity, respectively. Without the cascaded controller, the position controller would force the position to converge to the set point without regard for the velocity of the vehicle. The block diagram of the system setup is shown in Figure 32. 


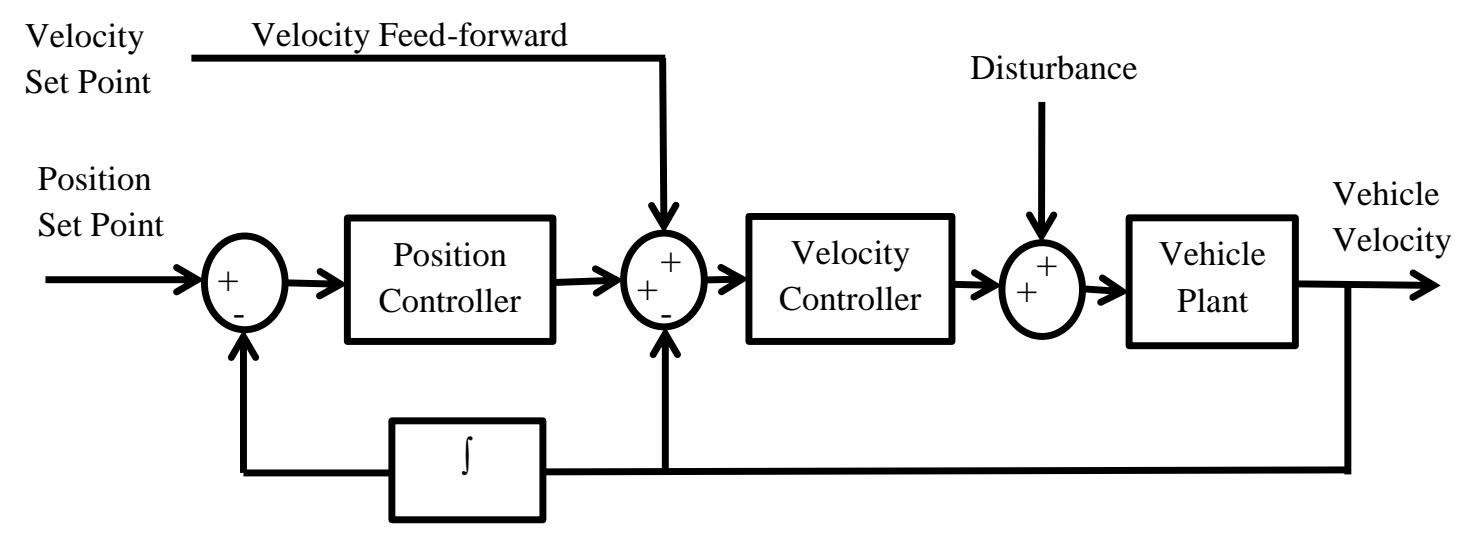

Figure 32. Block diagram of cascaded controller. The cascaded controller consists of an inside velocity control loop and an outside control loop. A velocity feed-forward loop is also implemented to improve the velocity response time.

An additional feature that was added in the controller was a velocity feed-forward loop. This loop approximates what the voltage input should be to the system based on the velocity set point. This value is then fed forward into the summation calculating the velocity error. This allows the system to anticipate how it needs to react based on upcoming circumstances. The values that were needed were approximated by measuring steady state speed at voltage set points throughout the velocity range of the vehicle.

The velocity of the cascaded controller was tuned by increasing the value of the proportional gain until the velocity started to oscillate. The gain that it started to oscillate was 0.8 and the system oscillated with a period of 0.4 seconds. This system response at this gain is shown in Figure 33. 


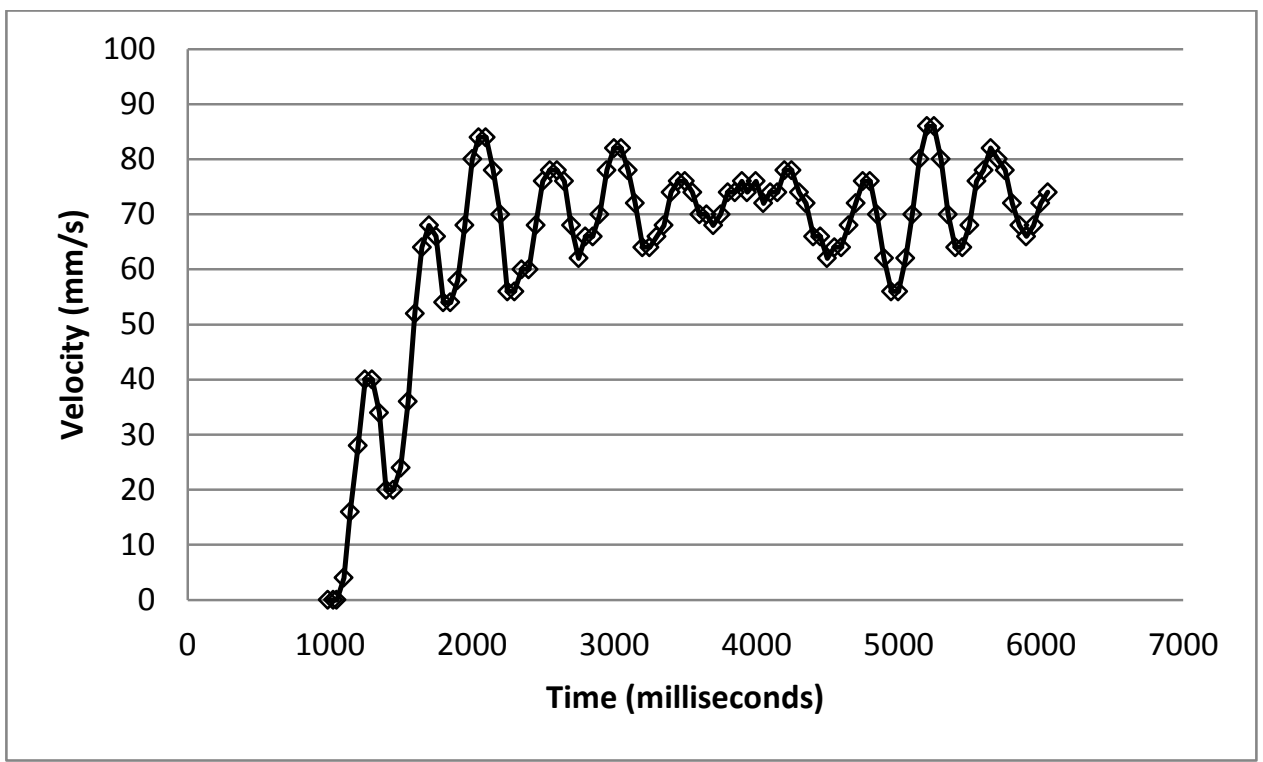

Figure 33. Zeigler-Nichols criterion data for velocity control. The velocity proportional gain of the system was increased until the system underwent sustained oscillations. The period of oscillation was used to determine the gains of the PID controller.

The Zeigler-Nichols criterion was then used to determine initial values to be used for the gains. The equations to determine the gains are shown in equations 49 through 51 (Ziegler \& Nichols, 1993).

$$
\begin{aligned}
& K_{p}=0.6 K_{u}=0.48 \\
& K_{i}=\frac{2 K_{p}}{T_{u}}=2.4 \\
& K_{d}=\frac{K_{p} T_{u}}{8}=0.024
\end{aligned}
$$

These values were used initially with the cascaded controller, but there was still a significant amount of overshoot with velocity as it hunted for the correct speed. The gains were tuned to decrease the overshoot and smooth the response. The optimal coefficients for the real world prototype were $\mathrm{K}_{\mathrm{p}}=0.3, \mathrm{~K}_{\mathrm{i}}=0.01$, and $\mathrm{K}_{\mathrm{d}}=0$. Additionally, the position controller coefficients were adjusted to $K_{p}=1.592, K_{i}=0.01$, and $K_{d}=0$. These 
results were determined by looking at the root locus of the closed loop system and testing the results on the prototype vehicle.

The first step in determining the optimum coefficients from the root locus was to minimize the integral gain while ensuring acceptable rise time for the system. This allowed the system to have low steady state error with a good rise time. The next step was to increase the derivative gains to improve rise time and setting time. However, after implementing the derivative control in the system, the derivative control increased the noise in the system and it sacrificed system robustness. Therefore, the derivative term was eliminated from the controller. The next step was to look at the root locus and adjust the proportional gains until just before the poles on the root locus diverged from the real axis.

The resulting transfer function of the position controller is shown in equation 52 where $\mathrm{E}$ is error. The output of the transfer function is a new velocity set point.

$$
\frac{\text { Velocity }(\mathrm{s})}{\mathrm{E}_{\text {position }}(\mathrm{s})}=1.592+\frac{.01}{\mathrm{~s}}
$$

The transfer function for the velocity controller is shown in equation 53.

$$
\frac{\text { Voltage }(\mathrm{s})}{\mathrm{E}_{\text {velocity }}(\mathrm{s})}=0.3+\frac{.01}{\mathrm{~s}}
$$

The resulting system transfer function is shown in equation 54.

$$
\frac{\Theta(s)}{\theta_{\text {setpoint }}(s)}=\frac{17.37 \mathrm{~s}^{2} 9+0.69 s+.0036}{0.61 s^{4}+6.57 s^{3}+17.56 s^{2}+.69 s+.0036}
$$

The root locus, pole zero map, and step response of the system were generated using Matlab and the code is shown in Appendix F. The root locus is shown in Figure 34. 


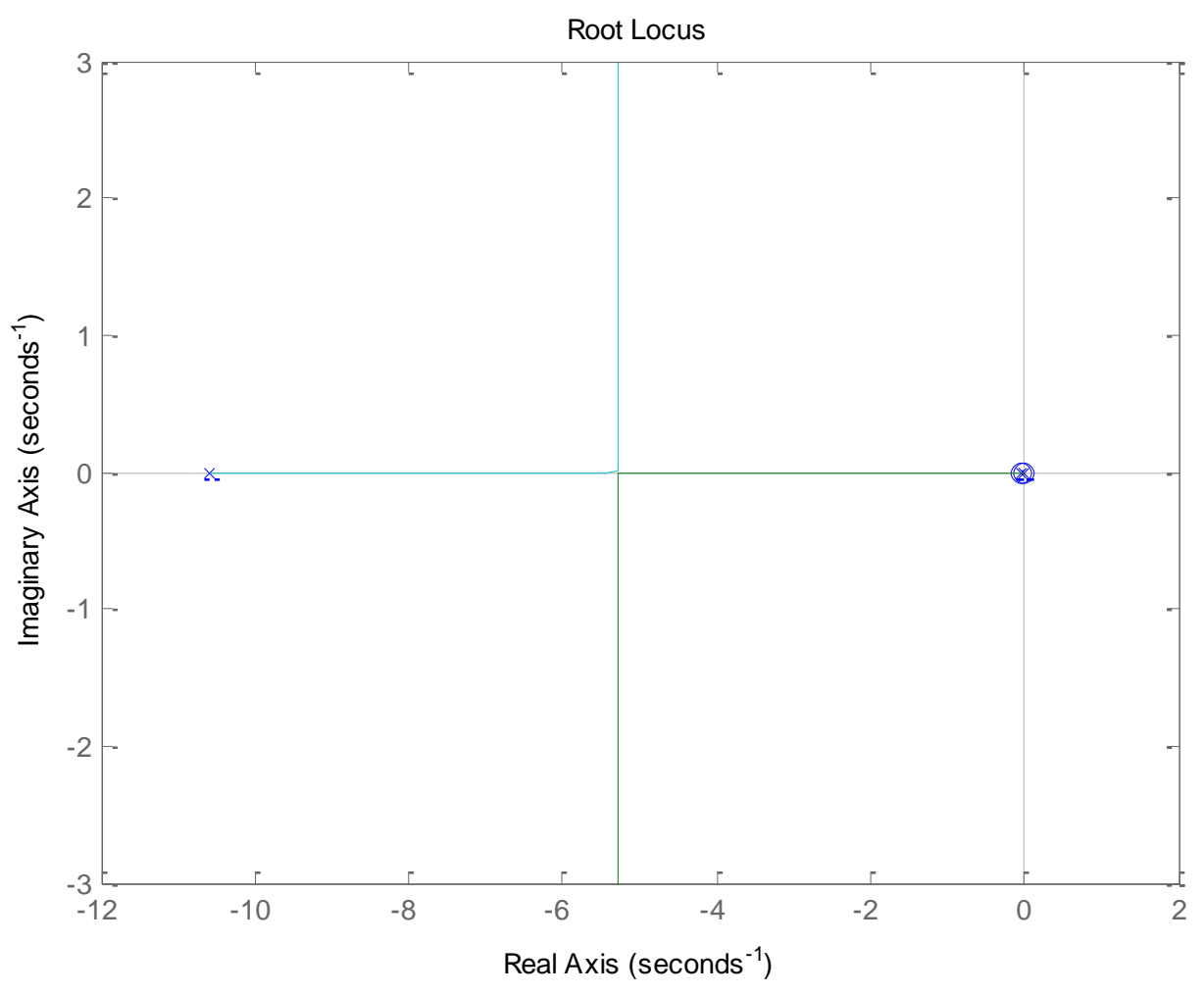

Figure 34. Root locus of cascaded controller. The root locus shows the location of the closed loop poles as the gain of the controller is increased from zero to infinity. The proportional gains were increased until just before the poles diverged from the real axis.

The root locus plot shows that there are two poles and two zeros very near the real axis. This was intentional because the pole placement near the real axis allows the steady state error to go to zero. The pole was placed near the real axis for both the velocity and position feedback loops. However, any pole near the real axis slows the response of the system. In order to counteract this effect, a zero was placed near each pole to cancel out the slowing effect of the pole. These results are shown in the closed loop pole and zero map in Figure 35. 


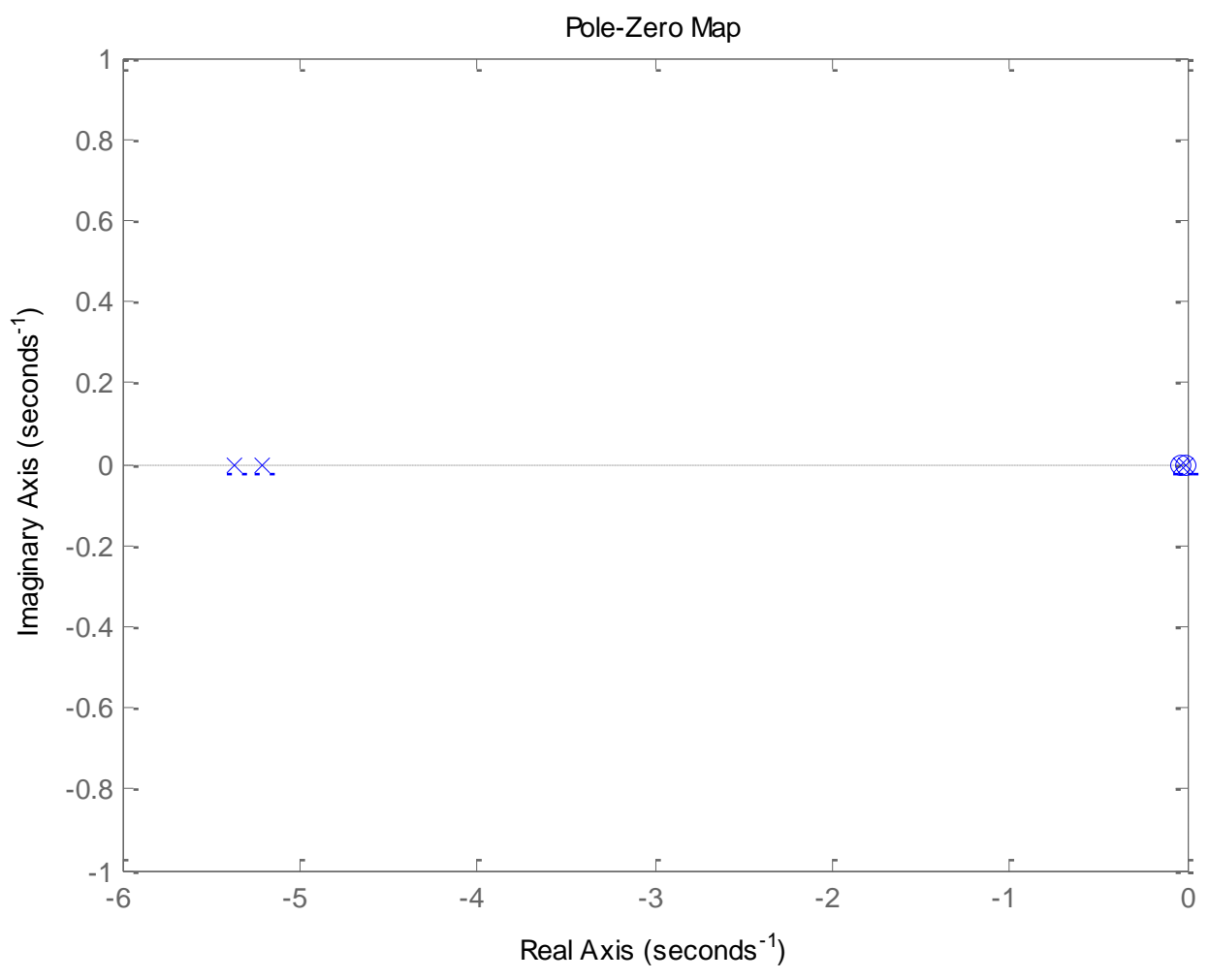

Figure 35. Closed loop pole and zero map of the cascaded controller. The poles of the system are indicated by crosses and the poles are indicated by circles.

The pole and zero map shows that there are poles near the real axis to eliminate steady state error, and there is a zero near each pole to cancel the slowing effect of the poles near the real axis. Additionally, the two other poles in the system were driven as far to the left as possible on the real axis by adjusting the gains of the controller. If the overall gain was adjusted any higher, the poles would leave the real axis, which would cause undesirable overshoot in the system. The theoretical step response of the closed loop system based on the model in equation 54 was created in Matlab. The response is shown in Figure 36. It shows that the response of the system is critically damped which gives optimum rise time with no overshoot. 


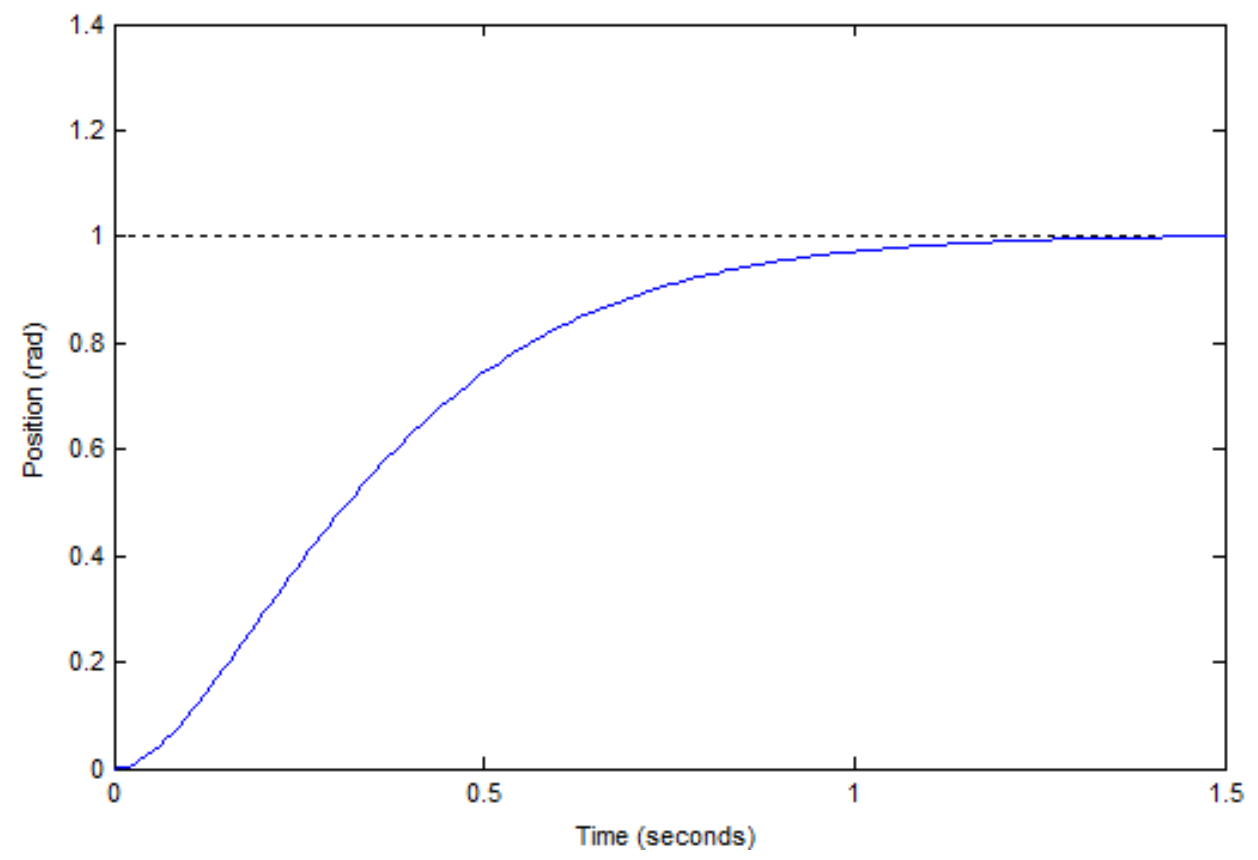

Figure 36. Theoretical step response of the cascaded controller. The graph shows the theoretical step response to a one volt input.

The vehicle position as it travels around the track is shown in Figure 37.

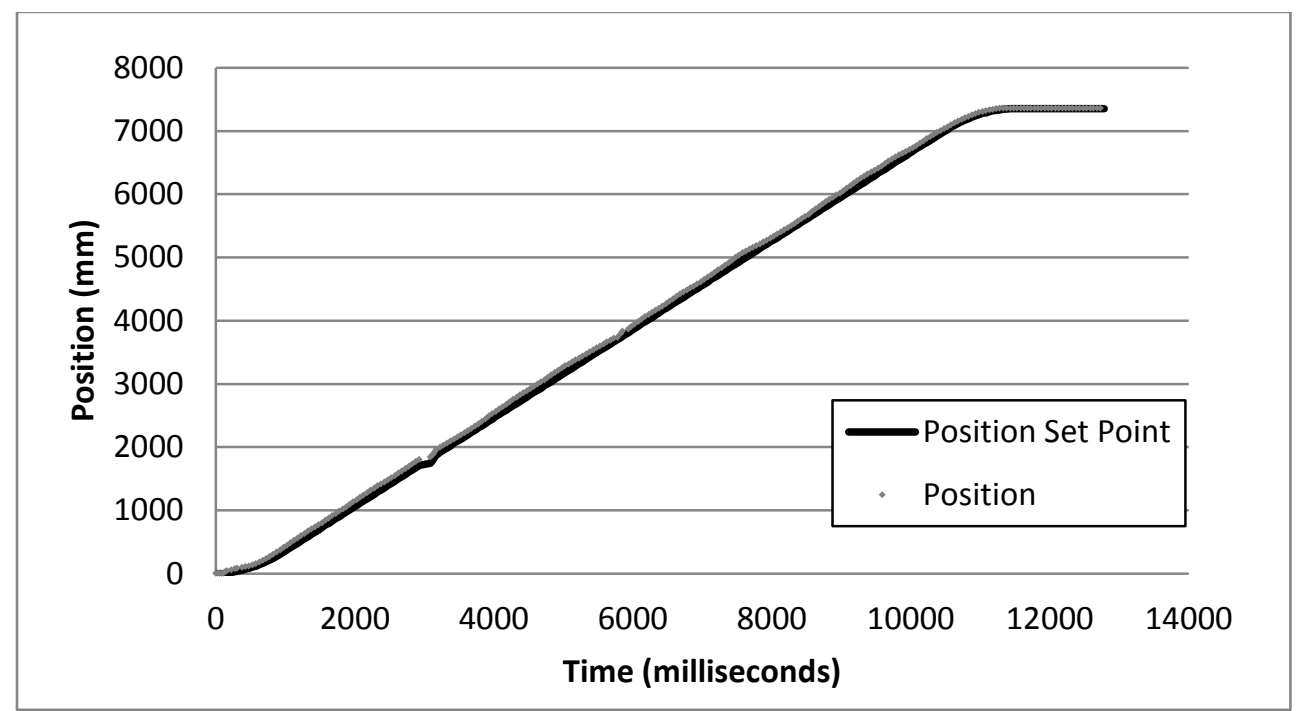

Figure 37. Position with cascaded position velocity controller. The graph shows the comparison of position set point verses actual position though one lap around the track.

The velocity of the vehicle as it traveled around the track is shown in Figure 38. 


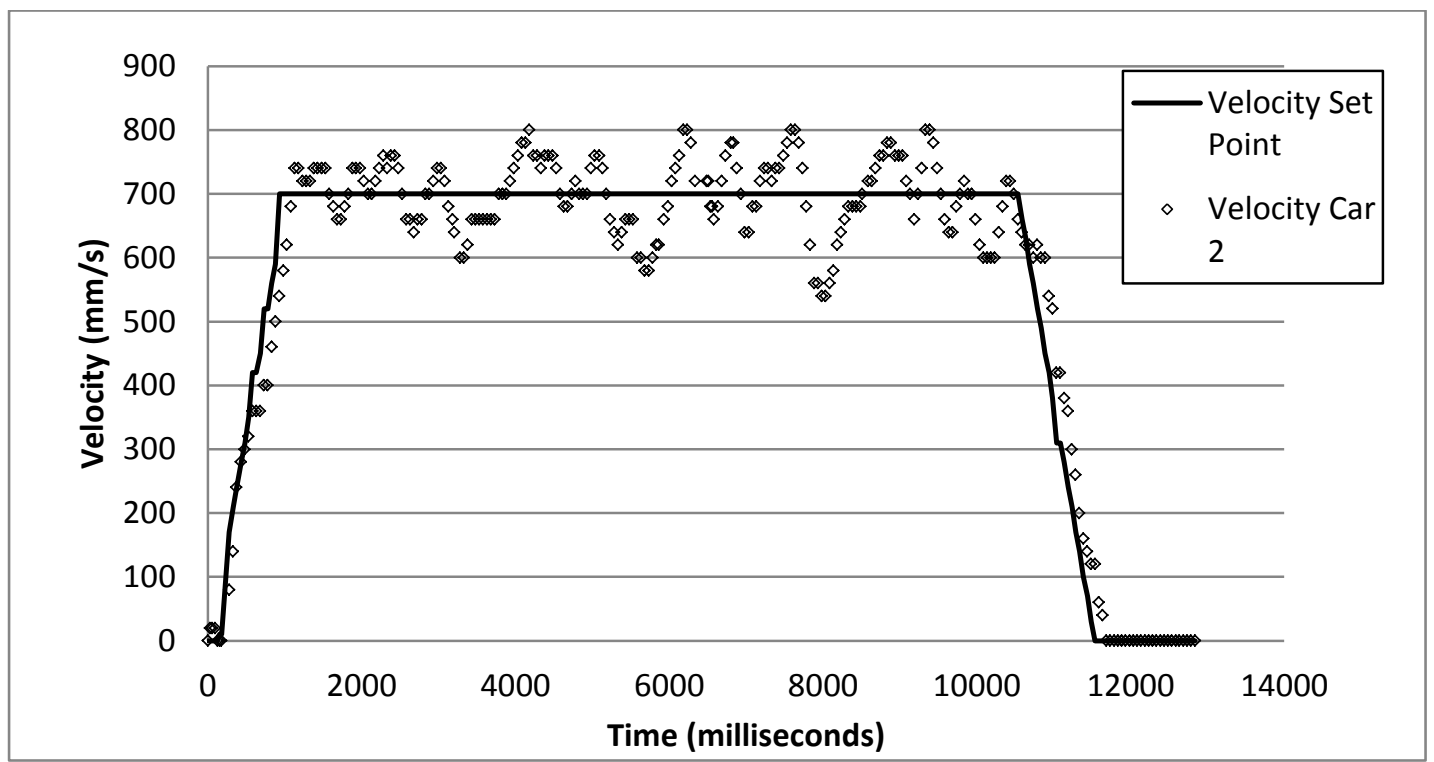

Figure 38. Velocity with cascaded position velocity controller. The graph shows the comparison of velocity set point verses actual position though one lap around the track.

The previous figures show the position tracks the correct set point within $40 \mathrm{~mm}$. The velocity also tracks the set point very well; however, the velocity is noisier than the position data. The velocity resolution can be calculated using equation 55 where $\mathrm{v}$ is actual velocity $(\mathrm{mm} / \mathrm{s}), \hat{v}$ is estimated velocity $(\mathrm{mm} / \mathrm{s}), \mathrm{R}$ is encoder resolution $(\mathrm{mm})$, and $\mathrm{jT}$ is the time between velocity estimates (ms) (Liu, 2002).

$$
\begin{aligned}
& |v-\hat{v}|=\frac{2 R}{j T} \\
& |v-\hat{v}|=\frac{2 * 3 \mathrm{~mm}}{50 \mathrm{~ms}} \\
& |v-\hat{v}|=120 \mathrm{~mm} / \mathrm{s}
\end{aligned}
$$

Equation 57 shows the possible velocity error in the track system. The noise in the velocity data can be accounted for since the velocity error could be as high as 120 $\mathrm{mm} / \mathrm{s}$. 
Additionally, friction is changing constantly as the vehicle travels around the track. The friction acts as a disturbance to the system and the control system is constantly reacting to it. The disturbance enters the system as an additional torque that the motor needs to overcome. This can be described by splitting the vehicle transfer function into an electrical transfer function and mechanical transfer function and adding the disturbance torque into the torque needed for the mechanical system. Alternatively, the disturbance can be converted into a voltage increase needed to produce the additional torque. The latter approach is shown in the block diagram in Figure 32.

\section{Multiple Vehicle System}

The overall system with multiple cars using the cascaded control system was characterized by putting two vehicles on the track. Both vehicles were programmed to accelerate to a maximum speed of $700 \mathrm{~mm} / \mathrm{s}$ and then decelerate to $0 \mathrm{~mm} / \mathrm{s}$. The position set point dictated that the vehicles stop when they completed exactly one lap. The vehicles' velocities are shown in Figure 39. 


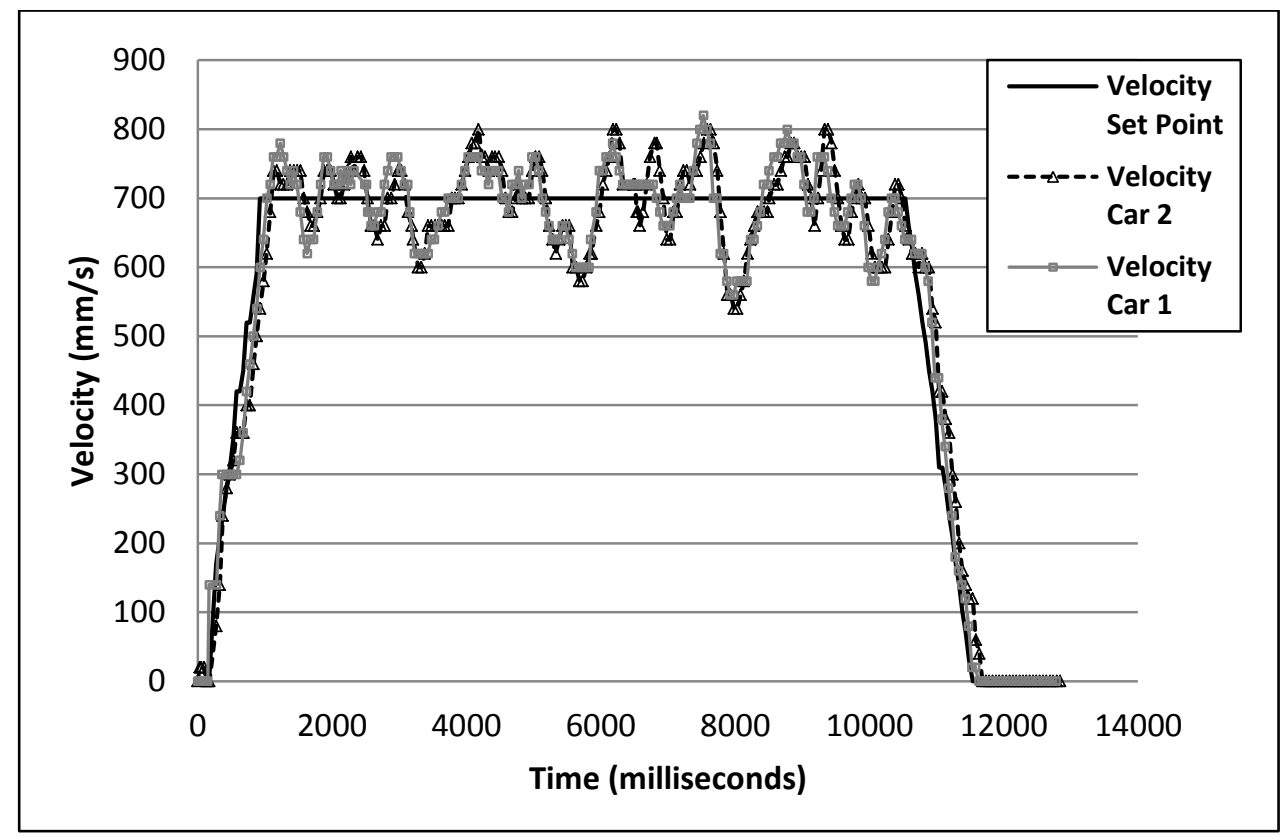

Figure 39. Velocity of two moving vehicles. The graph shows a comparison of the velocity of two moving vehicles that are controlled with position velocity cascaded controllers.

This figure shows that the velocity of the vehicles is changing relative to each other but they are both tracking the velocity set point. The positions track the set point position with $42 \mathrm{~mm}$ at all positions around the track and this can be seen in Figure 40 .

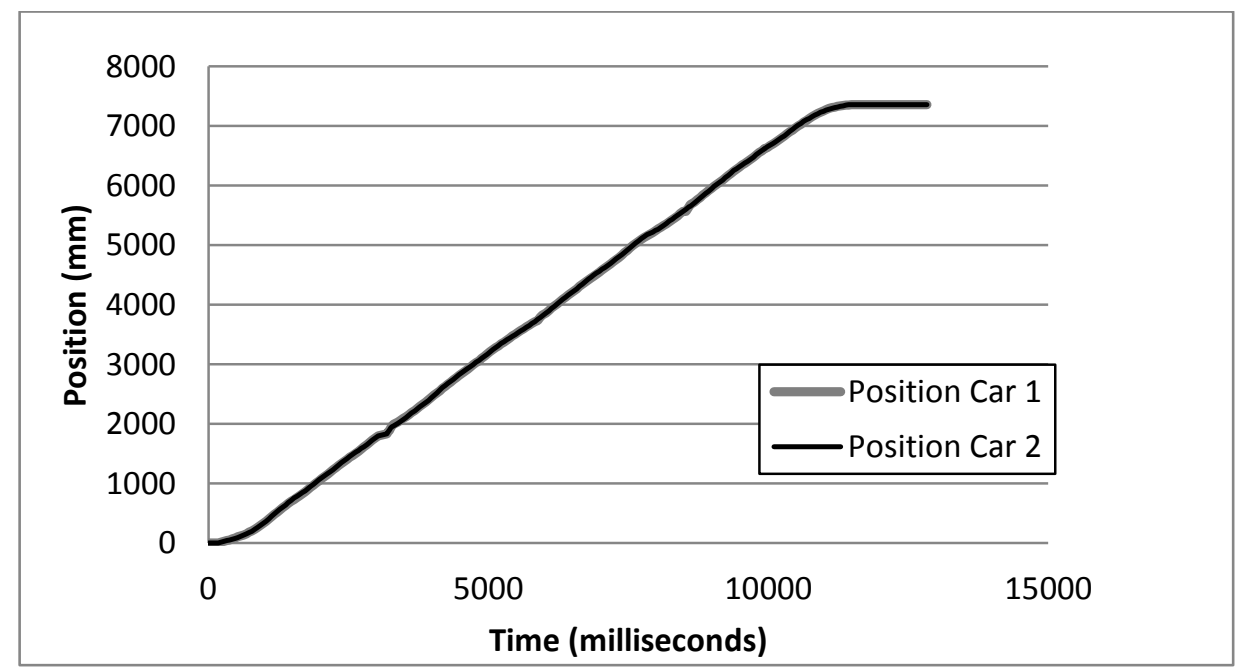

Figure 40. Position with two vehicles with cascaded controller. The graph shows a position comparison of two vehicles travelling with a cascaded controller. 
The relative vehicle position is also important because the vehicles cannot crash into one another. The difference between vehicle positions is shown in Figure 41. The vehicles started $100 \mathrm{~mm}$ apart. The data was normalized to $0 \mathrm{~mm}$ by subtracting $100 \mathrm{~mm}$ from the difference in position between the vehicles.

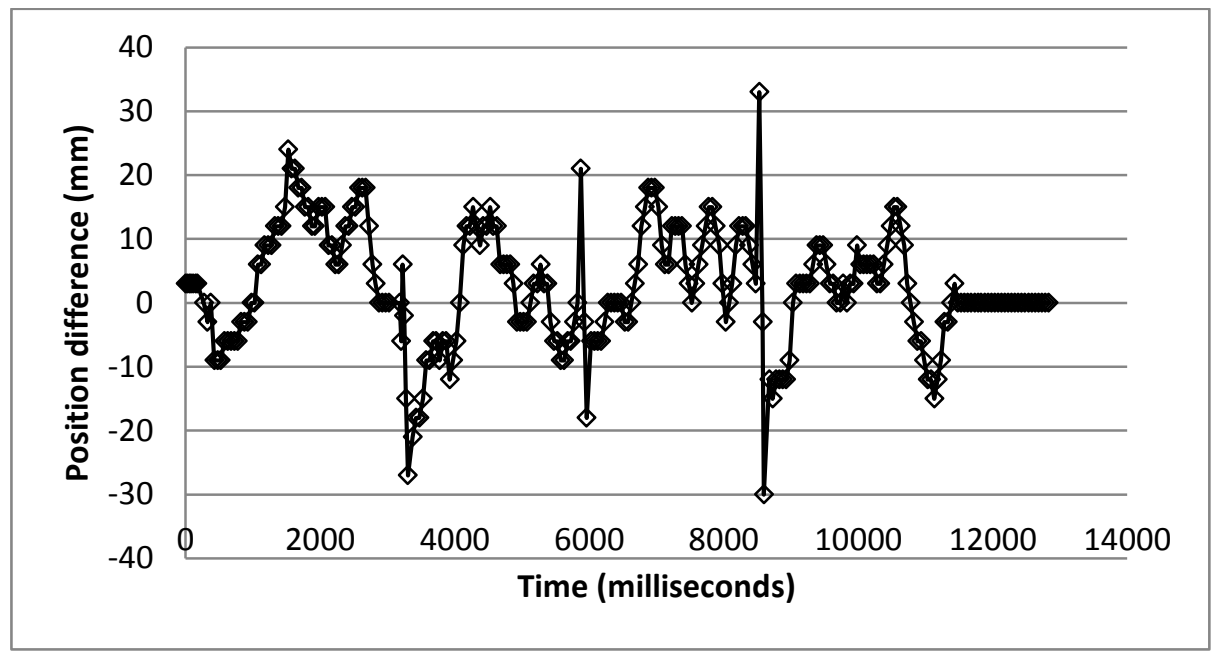

Figure 41. Difference in vehicle position. The graph shows the difference in position between two vehicles as they travel around the track. The data is normalized to $0 \mathrm{~mm}$.

Figure 41 shows that the position difference between the vehicle can vary as much as plus or minus $33 \mathrm{~mm}$ with an average difference of plus or minus $20 \mathrm{~mm}$. This corresponds to a full-scale difference in position of 0.96 meters. One method to decrease this error would be to fully characterize the friction on all tracks the vehicle could run on. The friction is consistent from vehicle to vehicle as it travels in different locations along the track. This is because the major source of friction is between the vehicle and the track. This friction data could then be used to feed-forward a voltage increase at the corresponding track position. 


\section{Chapter 4 Conclusions and Recommendations for Future Work}

The vehicle and track designed met the objective of the study. The design is a low cost, easily expandable system so that future development work can be done on the control systems governing ATN.

Special concern was taken in selecting parts that both met the objective of this study while minimizing cost. It is imperative that the cost of the platform is low so that track infrastructure as well as vehicle fleet size can be expanded. This allows a variety of potential ATN concepts to be tested in real time. The vehicle was designed to be manufactured with a three-dimensional printer. This was a low-cost solution because of the availability of the printer. However, the vehicle design could easily be altered so that it can be manufactured on a milling machine. Alternatively, the design could be adjusted so that the parts could be cheaply molded if volume requirements are high enough. Either of these options could be an area for future work if three-dimensional printing is not an option for manufacturing.

It is also recommended that in future work a printed circuit board assembly (PCBA) is designed and manufactured for this vehicle. A printed circuit board would save size, cost, and improve robustness. The microcontroller, XBEE shield, and motor driver could all be incorporated into a single PCBA. The components of the PCBA would be significantly less than the cost of buying each component individually. It is estimated that the cost could be reduced by approximately $\$ 50$ by implementing a PCBA.

Overall the track assembly was low cost, easy to assemble, and easy to manufacture. Its performance could be improved by ensuring that each track section is 
supported at least every $400 \mathrm{~mm}$. After assembling the track, it was determined that the track is flexible if it is supported less than $400 \mathrm{~mm}$. Alternatively, the thickness of the sheet metal could be increased to increase its stiffness. However, the material and manufacturing cost would need to be taken into account before increasing the thickness.

The cascaded control scheme used in this implementation balances the need for accurate position with maintaining velocity control. This could be improved by improving the resolution of the velocity estimation. One way to do this would be to increase the resolution of the linear encoder. Additionally, the control system could be improved by reducing the friction between the track and the vehicle. A simple upgrade to decrease friction would be to include a roller in the switching mechanism.

The design of the cascaded controller allows a master controller to be implemented right on top of the cascaded controller. In fact, the system was designed so that future work on the master controller would be easy to implement. This architecture allows the ability to test many master control systems while not having to design the individual control system for each vehicle.

In future work, the master controller could be updated to include a controller based on vehicle separation. The current master controller uses set points to tell vehicles where to go in the system. However, there is no feedback system preventing accidents if the master controller makes an error or a vehicle breaks down in the guideway. Therefore, it is recommended that the next steps on this project are to implement a master controller that takes into account separation distances between vehicles. For example, the controller would start if the separation distance between vehicles went below a 
specified threshold. The controller would use the vehicle position to calculate vehicle separation distance and use this as a feedback mechanism. A master controller such as this would be more robust and safe because the vehicles would react if a vehicle broke down in the track guideway to avoid a collision.

Since the beginning of ATN, there has been a substantial amount of theoretical research and full-scale testing, but there are few scaled physical models that have been developed to test ATN concepts. The system described here will allow researchers to further develop control systems and study system operation in order to evaluate and validate specific questions regarding the implementation of an ATN system. The modeling approach shown has potential to allow benefits of ATN to be realized and concerns to be addressed allowing the world to come closer to an environmentally responsible, economical solution to transportation that passengers will appreciate. 


\section{References}

2getthere. (2012, July). Masdar PRT Application. Retrieved October 24, 2013, from Sustainable Mobility Solutions: http://www.2getthere.eu/?page_id=10

Aerospace Corporation. (2012). Automated Transit Network Feasibility Evaluation San José Mineta International Airport San José, CA. El Segundo: Aerospace Corporation.

Aerospace Corporation. (2014). Aerospace. Retrieved April 26, 2014, from https://www.aerospace.org/

Anderson, J. (1984). Optimization of Transit-System Characteristics. Journal of Advanced Transportation, 18(1), 77-111.

Anderson, J. (2000). A Review of the State of the Art of Personal Rapid Transit. Journal of Advanced Transportation, 34(1), 3-29.

Anderson, J. (2005). The future of high-capacity personal rapid transit. Presentation at the Advanced Automated Transit Systems Conference. Bologna, Italy.

Austin, M. B. (2001). Building a case for a new transportation system. Retrieved from http://escholarship.org/uc/item/3fn7j6d2

Carnegie, J., \& Voorhees, A. (2007). Viability of Personal Rapid Transit in New Jersey. New Jersey Department of Transportation.

Digi. (2014, March 10). XBee RF Modules. Retrieved March 27, 2014, from Digi: http://www.digi.com/products/wireless-wired-embedded-solutions/zigbee-rfmodules/zigbee-mesh-module/

Fairchield Semiconductor. (2009, September). QRE1113 - Reflective Object Sensor. Retrieved October 20, 2013, from Fairchild: www.fairchildsemi.com

Helmer, J. R. (2009, March 27). Airport Area Automated Transit Network. San Jose, California: Santa Clara Transportation Authority.

Irving, J. H. (1978). Fundamentals of Personal Rapid Transit. Lexington, MA: D.C. Heath and Company.

Kimberly Horn and Associates, Inc. (2010). Assessment of New Tecchnologies: Personal Rapid Transit. Fresno: Fresno Council of Governments. 
Krueger, A. (2014, March 28). Automated Transit Network (ATN) prototype [Video file]. Retrieved March 28, 2014, from YouTube: http://youtu.be/K-feorJYHNI

Liu, G. (2002). On velocity Estimation Using Position Measurements. American Control Conference, (pp. 1115-1120). Anchorage, AK.

Lowson, M. V. (2011). Sustainable Personal Transportation. Forum on Integrated and Sustainable Transportation Systems (pp. 261-267). Vienna, Austria: IEEE.

Magnovate Technologies. (2013). Magnovate Technologies Realizing the Promise of Future Transportation Systems. Edmonton, Alberta, Canada.

McDonald, S. S. (2013). Personal Rapid Transit and Its Development. Transportation Technologies for Sustainability, 831-850.

Movellan, J. (2013, October 15). DC Motors. Retrieved March 20, 2014, from Machine Perception Laboratory: http://mplab.ucsd.edu/tutorials/DC.pdf

Muller, P. J. (2010). PRT Vendor Updates. PRT News and Views.

Pemberton, M. (2013). The Track to Suncheon: Making APMs Intelligent. Automated People Movers and Transit Systems, 236-275.

Schneider, J. (2012, September 20). Cabintaxi PRT System. Retrieved January 20, 2014, from Innovative Transportation Technologies: https://faculty.washington.edu/jbs/itrans/cabin.htm

Sproule, William J., \& Edward S. Neumann. (1991). The Morgantown PRT: It Is Still Running at West Virginia University. Journal of Advanced Transportation, 269279.

Taxi 2000. (2010). Skyweb Express transportation for the 21 st Century. Retrieved October 1, 2013, from http://faculty.washington.edu/jbs/itrans/SWE\%20marketing\%20intro.pdf

Taxi 2000. (2013). Skyweb Express. Retrieved November 1, 2013, from Skyweb Express Technology: http://www.taxi2000.com/technology.html

The Engineering ToolBox. (2014, March 27). Drag Coefficient. Retrieved March 29, 2014, from The Engineering ToolBox: http://www.engineeringtoolbox.com/dragcoefficient-d_627.html 
Transport Innovators. (2013, August 1). Why no decent scale models of PRT using off the shelf robots? (Izmir Institute of Technology PRT Demostration project). Retrieved August 30, 2013, from Transport Innovators Forum:

https://groups.google.com/forum/\#!topic/transportinnovators/ikdYsIDWCTc\%5B1-25-false\%5D

Ultra Global. (2013, November 28). ultra pods. Retrieved November 20, 2013, from Ultra Global PRT: http://www.ultraglobalprt.com/how-it-works/ultra-vehicle/

United States. (1964). Urban Mass Transportation Act of 1964, Pub. L. No. 88-365 § 78, 100 Stat. 302.

Vectus. (2013). Vectus Intelligent Transport. Retrieved November 10, 2013, from Vectus: http://www.vectus.com

Vectus Ltd. (2012). Media Gallery. Retrieved November 20, 2013, from Vectus Intelligent Transport: http://www.vectusprt.com/EN/media/images/

Ziegler, \& Nichols. (1993). Optimum Settings for Automatic Controllers. Journal of Dynamic Systems, Measurement, and Control, 759-768. 


\section{Appendix A}

\section{System Design Requirements}

\section{General}

Develop a low-cost, easily portable, simplified, small scale Automated Transportation Network (ATN) to be used for the demonstration of some of the basic concepts of ATN transportation systems. This project will also be used for transportation network and vehicle control development. The ATN shall demonstrate the following:

a. Origin to destination service such that simulated trip requests at a station will initiate a vehicle traveling from the trip request station to the selected destination station. If a vehicle is not present at the origin station, an 'empty' vehicle will be sent to that station.

b. Non-stop vehicle service. Vehicles will not stop at intermediate stations between the origin station and a destination station.

c. Offline stations allowing vehicles to pass as passengers embark and disembark. The ATN will not have offline parking of vehicles. However, the system will be designed to allow for this in a future expansion if desired.

d. Data will be collected on trip requests per unit time (minute), time between trip request and vehicle departure, and time between vehicle departure and vehicle arrival. The goal will be to minimize the time between trip requests and vehicle departure.

e. System capacity by continually adding vehicles until performance starts to degrade, i.e. vehicle movements begin to interfere with each other. 
f. Comparison of results to theoretical models and simulations.

\section{ATN Controller}

The ATN will have a master controller that will do the following:

a. Control routing and movements of all vehicles.

b. Maintain location information on all vehicles.

c. Display vehicle location information in a graphical user interface display. This will include a layout of the system and location of the vehicles in it.

d. Allow users to set up and design different scenarios of trip generation, including symmetrical, asymmetrical, random, and manual trip requests.

e. Display number of passengers at stations who have requested vehicles.

f. Allow capability to alter the headway criteria used by the ATN to show how this affects system capacity as well as safety concerns in case of rapid deceleration.

g. Display a layout of the demonstration track and all conflict points such as diverges, merges, and station stops.

h. Allow operators to 'initialize' vehicles as they are introduced onto the track such that it enters the location of new vehicles into its inventory.

\section{Stations}

The stations will have the following characteristics:

a. The stations will be offline with diverges and merges to allow vehicles to exit and enter the mainline. Vehicles stopped in stations will not impede mainline traffic flow when they stop to allow passengers to embark and disembark the vehicle. 
b. Stations will have adequate length ramps to allow vehicles to stop at berthing position(s).

c. Stations will have adequate length ramps to allow vehicles to accelerate to merge with mainline traffic.

d. Vehicles will not be able to leave the track at any point through the stations.

e. The stations will have visual displays to indicate the passenger queue count.

f. The stations will have a physical means to allow users to generate a trip request from that station to any one of the other stations.

\section{Vehicles}

a. Vehicles will be autonomous with an onboard controller such that they will be capable of making the trip without input from an outside controller, i.e. store track layout and routing information.

b. Vehicles will maintain onboard current location in relation to the overall track.

c. Vehicles will have the capability to periodically read a track mounted landmark and update its current position information accordingly.

d. Vehicles will follow a preset track guide way from one destination to another.

e. Vehicles will maintain a following distance from a vehicle in front to avoid vehicle collisions.

f. Vehicles will maintain a preset velocity.

g. Vehicles will switch tracks as appropriate to reach the final destination.

h. Vehicles will display total passengers occupying the vehicle. 
i. Vehicles will be battery powered and maintain a charge for at least five minutes of continuous use.

5. Track Layout

a. The track will be an oval shape with at least four offline stations.

b. The track will not exceed an $8 \mathrm{ft} \times 8 \mathrm{ft}$ footprint and be constructed so as to be easily assembled and disassembled.

c. The track will not have in track switching.

d. The track will provide guidance for the vehicles so that vehicles will easily stay within the track.

e. The track will have a vehicle initialization area where vehicles will be introduced into the ATN.

\section{Communications Network}

The communications network will provide communications between the vehicles and ATN controller.

a. The communications network will be robust enough to communicate with at least 10 vehicles.

b. The communication network will reliably transfer route requests to vehicles.

c. The communication network will allow the vehicles to send location information to the ATN controller and be used by the ATN to communicate the location of conflict or stopping locations to each vehicle.

d. The communication network will relay quantity of passenger information to the vehicle. 
e. The communication network will relay passenger queue information to each station.

7. Overall hardware and software design requirements

a. The vehicles and track they run on are to be designed from scratch using commercial off-the-shelf hardware components as well as custom manufactured pieces. Custom pieces will be manufactured using a three-dimensional printer.

b. The ATN controller will be a commercially available computer.

c. The onboard vehicle controller will be a commercially available microcontroller for robotic applications.

d. The software language will be $\mathrm{C}$ for the onboard vehicle controllers.

\section{System Implementation}

Due to the large nature of this project, the system will be implemented in stages with subgroups each tackling specific tasks. There will be meetings as necessary to coordinate the activities of each sub-group. All work and designs will be documented and be reviewed within the group at a minimum. The design documentation will provide information on design philosophies as well as detailed information, i.e. why certain design approaches were taken, what those approaches were, and how they were implemented.

Phase 1: A test track and three vehicles will be developed for feasibility testing of the system. This will include development of both computer aided drafting (CAD) files and a working prototype that can be tested. The prototype will contain adequate sensors to control the vehicle by specific position, velocity, and acceleration profiles. 
Additionally, the prototype will have the ability to maintain specific headways. Preliminary results will be adequately documented to show the performance of the system and the ability to expand.

Phase 2: A master controller will be fully developed to perform coordinated movements of the vehicles which conform to an overall master system approach. The master controller will have functionality to control the system with multiple simulated passenger demand scenarios, including symmetric, asymmetric, and random. The system will have a meaningful graphical user interface that shows the operation of the system and has data export capability for further analysis. The communication protocol will be improved so that the system can be expanded until system degrades. Communication protocol should not be the weakest link. The test track will be improved to contain a visual display that shows the number of people waiting in the queue at each station.

Phase 3: Expand the project to test system degradation. Maximum capacity is determined by the maximum number of vehicles that can operate on the system without interfering with each other, vehicle transit times, and passenger wait times. Multiple control algorithms should be designed and implemented at this phase to show how the system reacts under different control algorithms in different loading scenarios. Additionally, mixed mode operation with different size vehicles can be used to show the effect of mixed mode operation. 


\section{Appendix B}

\section{Bill of Materials of the Track Assembly}

\begin{tabular}{|l|l|l|l|l|l|}
\hline $\begin{array}{l}\text { Item } \\
\text { Number }\end{array}$ & Description & Quantity & $\begin{array}{l}\text { Unit } \\
\text { Cost }\end{array}$ & $\begin{array}{l}\text { Total } \\
\text { Cost }\end{array}$ & $\begin{array}{l}\text { Source } \\
\text { (Part Number) }\end{array}$ \\
\hline 1 & $\begin{array}{l}\text { \#8 x 0.5in Zinc- } \\
\text { Plated Screws }\end{array}$ & 64 & $\$ 0.08$ & $\$ 5.12$ & $\begin{array}{l}\text { Lowes } \\
(59177)\end{array}$ \\
\hline 3 & $\begin{array}{l}\text { 3/4 x 48 x 96 in } \\
\text { Industrial } \\
\text { Particleboard }\end{array}$ & 2 & $\$ 20.42$ & $\$ 40.84$ & $\begin{array}{l}\text { Lowes } \\
(12260)\end{array}$ \\
\hline 4 & $\begin{array}{l}\text { Gatehouse 4-in } \\
\text { Door Hinge }\end{array}$ & 3 & $\$ 0.30$ & $\$ 0.90$ & $\begin{array}{l}\text { Lowes } \\
\text { (308921) }\end{array}$ \\
\hline 5 & $\begin{array}{l}\text { Track corner2 } \\
\text { out }\end{array}$ & 8 & $\$ 3.32$ & $\$ 26.56$ & Solidworks Module \\
\hline 6 & $\begin{array}{l}\text { Track corner1 } \\
\text { in }\end{array}$ & 8 & $\$ 7.55$ & $\$ 60.40$ & Solidworks Module \\
Track corner1 & 4 & $\$ 7.63$ & $\$ 30.52$ & Solidworks Module \\
\hline 7 & Track mirror & 4 & $\$ 8.20$ & $\$ 32.80$ & Solidworks Module \\
\hline 8 & $\begin{array}{l}\text { Track straight } \\
\text { long }\end{array}$ & 4 & $\$ 3.05$ & $\$ 12.20$ & Solidworks Module \\
\hline 9 & $\begin{array}{l}\text { Track straight } \\
\text { out }\end{array}$ & 4 & $\$ 3.00$ & $\$ 12.00$ & Solidworks Module \\
\hline 10 & Tracksplit & 4 & $\$ 8.20$ & $\$ 32.80$ & Solidworks Module \\
\hline Total & & & & $\$ 254.14$ & \\
\hline $\begin{array}{l}\text { Total/foot } \\
\text { of track }\end{array}$ & & & $\$ 7.70$ & \\
\hline
\end{tabular}

The cost of custom parts was estimated using the Solidworks Costing Module. The program has a costing module that will estimate the cost of a custom part based on machining time and material cost. 


\section{Appendix C}

Bill of Materials for the Vehicle Assembly

\begin{tabular}{|c|c|c|c|c|c|}
\hline $\begin{array}{l}\text { Item } \\
\text { Number }\end{array}$ & Description & Quantity & $\begin{array}{l}\text { Unit } \\
\text { Cost }\end{array}$ & Total Cost & $\begin{array}{l}\text { Source } \\
\text { (Part Number) }\end{array}$ \\
\hline 1 & Chassis & 1 & $\$ 20.02$ & $\$ 20.02$ & Solidworks Module \\
\hline 2 & Switch & 2 & $\$ 1.55$ & $\$ 3.10$ & Solidworks Module \\
\hline 3 & Flexure & 4 & $\$ 0.96$ & $\$ 3.84$ & Solidworks Module \\
\hline 4 & Motor Mount & 1 & $\$ 4.50$ & $\$ 4.50$ & Solidworks Module \\
\hline 5 & Wheel & 8 & $\$ 0.50$ & $\$ 4.00$ & Solidworks Module \\
\hline 6 & Shaft $3 \mathrm{~mm}$ & 1 & $\$ 2.75$ & $\$ 2.75$ & $\begin{array}{l}\text { Mcmaster-Carr } \\
\text { (8920K14) }\end{array}$ \\
\hline 7 & Shaft $1 / 8$ in & 1 & $\$ 1.75$ & $\$ 1.75$ & $\begin{array}{l}\text { Mcmaster-Carr } \\
\text { (8279T16) }\end{array}$ \\
\hline 8 & Worm Gear & 1 & $\$ 2.50$ & $\$ 2.50$ & $\begin{array}{l}\text { Gizmozone } \\
\text { (gw0.5-01014) }\end{array}$ \\
\hline 9 & Worm & 1 & $\$ 2.20$ & $\$ 2.20$ & $\begin{array}{l}\text { Gizmozone } \\
\text { (gwg0.5-26-31) }\end{array}$ \\
\hline 10 & $\begin{array}{l}\text { Arduino Uno } \\
\text { R3 }\end{array}$ & 1 & $\$ 29.95$ & $\$ 29.95$ & $\begin{array}{l}\text { Sparkfun } \\
\text { (DEV-11021) }\end{array}$ \\
\hline 11 & XBEE Shield & 1 & $\$ 24.95$ & $\$ 24.95$ & $\begin{array}{l}\text { Sparkfun } \\
\text { (WRL-10854) }\end{array}$ \\
\hline 12 & XBEE Series 2 & 1 & $\$ 20.95$ & $\$ 20.95$ & $\begin{array}{l}\text { Sparkfun } \\
\text { (WRL-10414) }\end{array}$ \\
\hline 13 & $\begin{array}{l}11.1 \mathrm{~V} \text { Lithium } \\
\text { Ion Battery }\end{array}$ & 1 & $\$ 19.55$ & $\$ 19.55$ & $\begin{array}{l}\text { Hobby Partz } \\
\text { (32P-1500mAh- } \\
\text { 3S1P-111-20C) }\end{array}$ \\
\hline 14 & $\begin{array}{l}\text { QRE1113 Line } \\
\text { Sensor }\end{array}$ & 3 & $\$ 2.95$ & $\$ 8.85$ & $\begin{array}{l}\text { Sparkfun } \\
\text { (ROB-09454) }\end{array}$ \\
\hline 15 & $\begin{array}{l}\text { SN754410 } \\
\text { Half-H Driver }\end{array}$ & 1 & $\$ 2.35$ & $\$ 2.35$ & $\begin{array}{l}\text { Sparkfun } \\
\text { (COM-00315) }\end{array}$ \\
\hline 16 & Scalextric Kit & 3 & $\$ 2.06$ & $\$ 6.18$ & Scalextric (C8523) \\
\hline Total & & & & $\$ 157.44$ & \\
\hline
\end{tabular}

The cost of custom parts was estimated using the Solidworks Costing Module. The program has a costing module that will estimate the cost of a custom part based on machining time and material cost. 


\section{Appendix D}

\section{Drawings of Custom Parts}

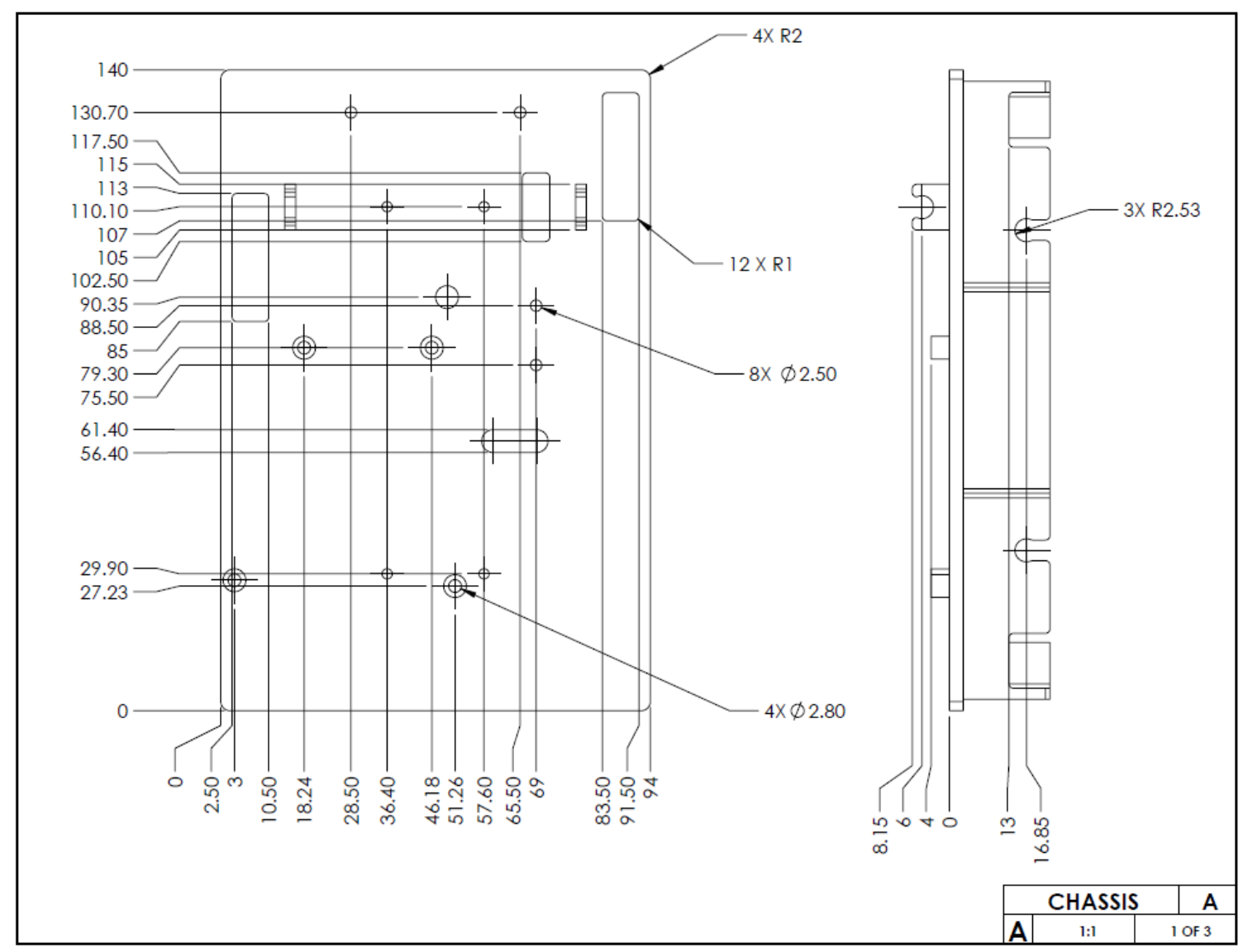




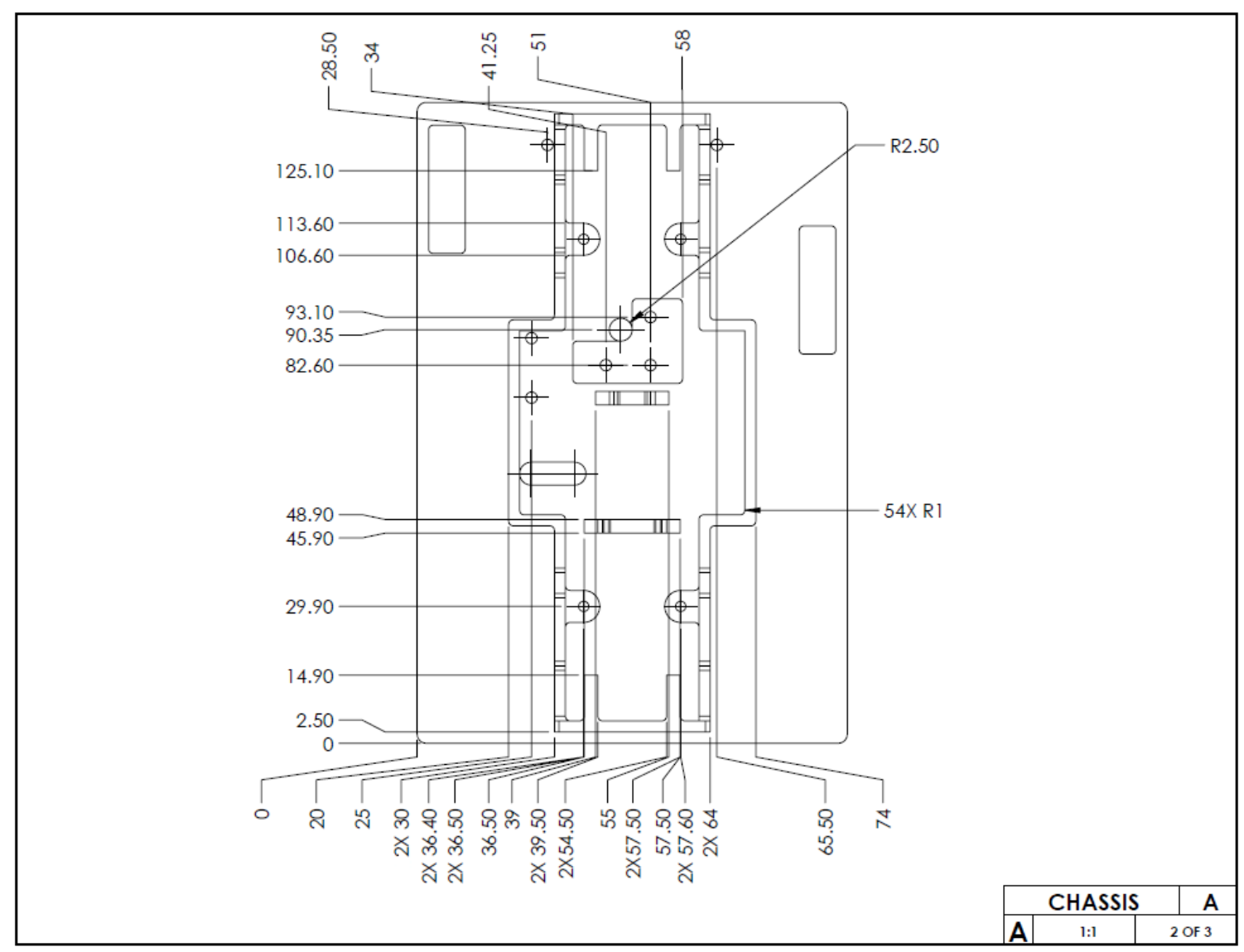




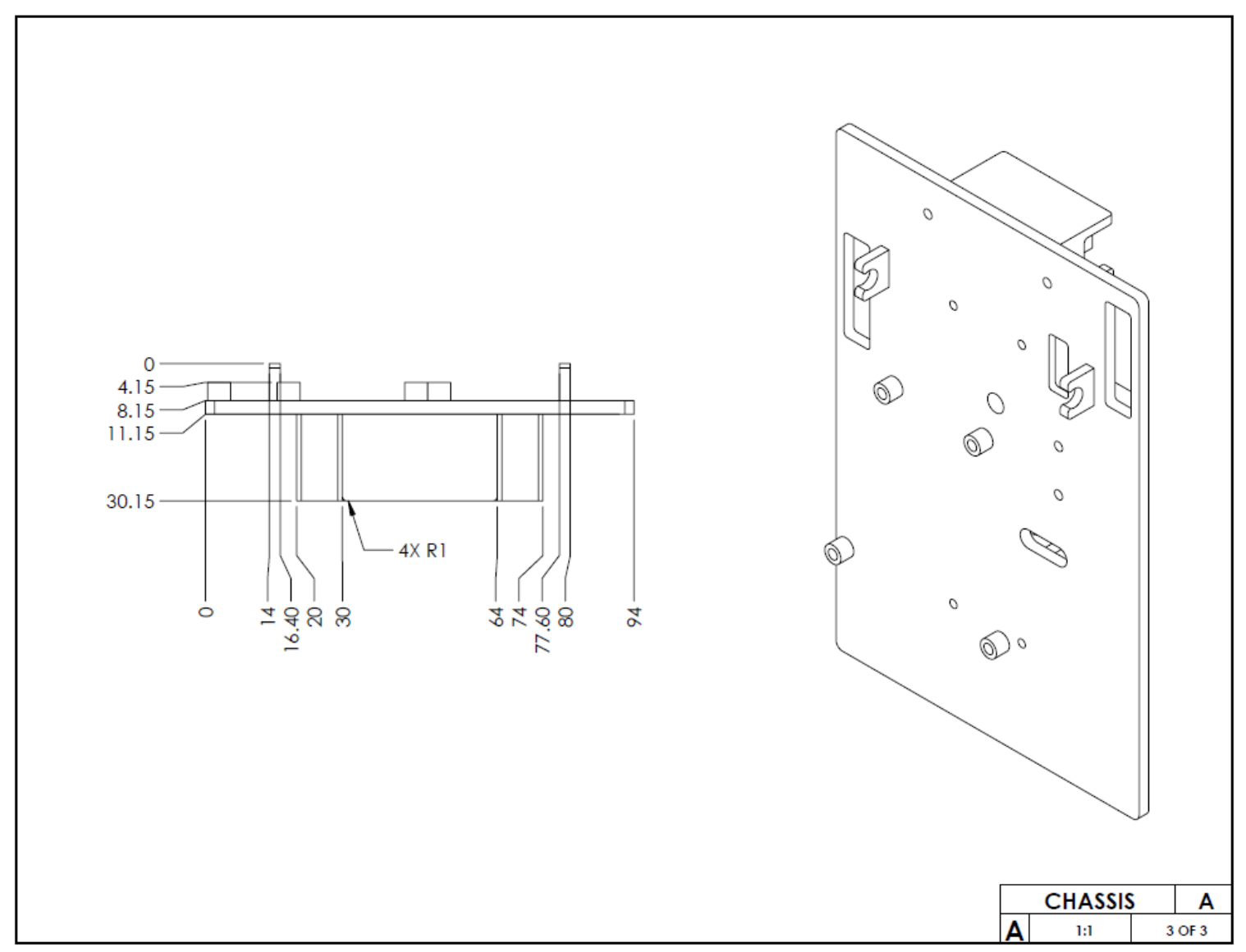




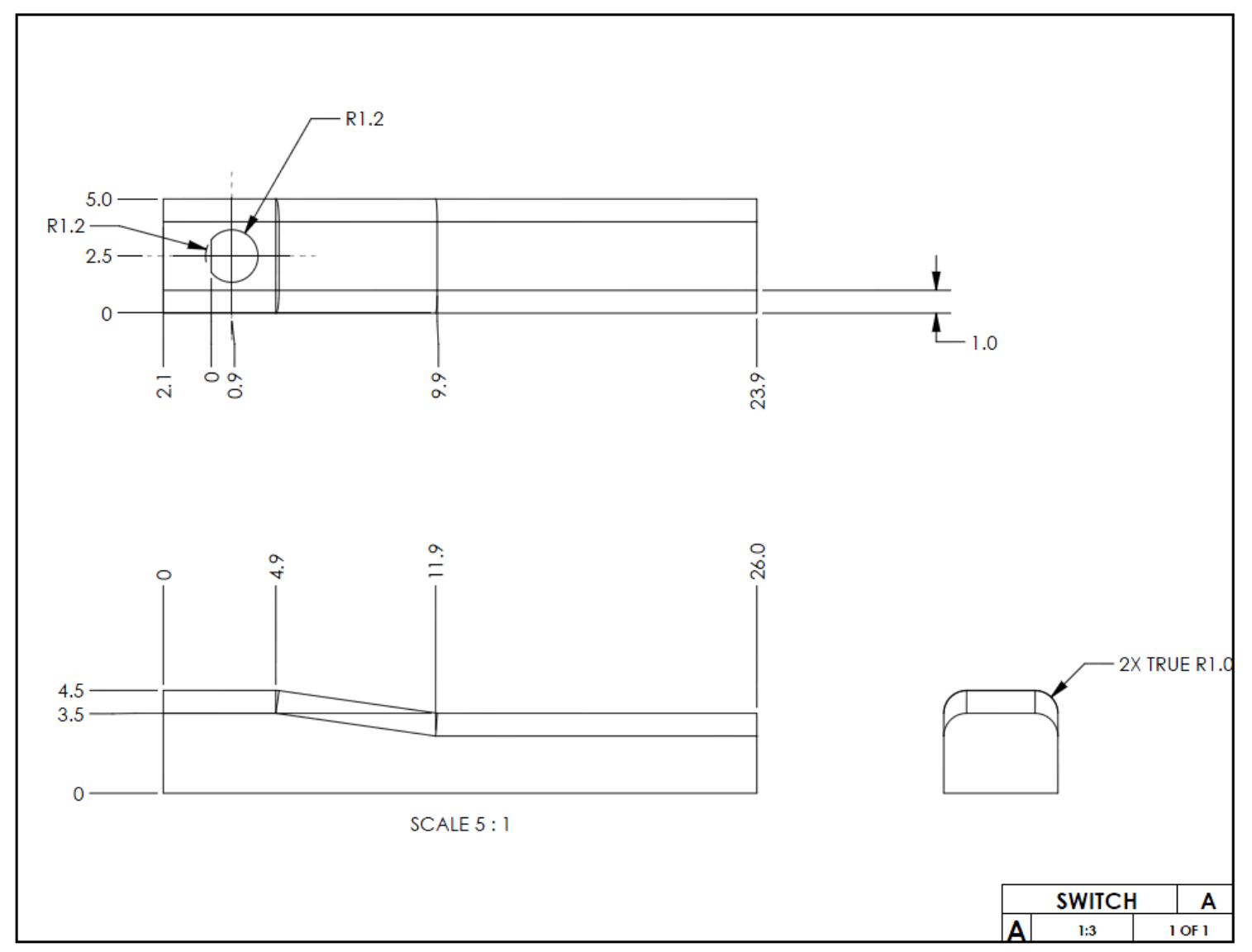




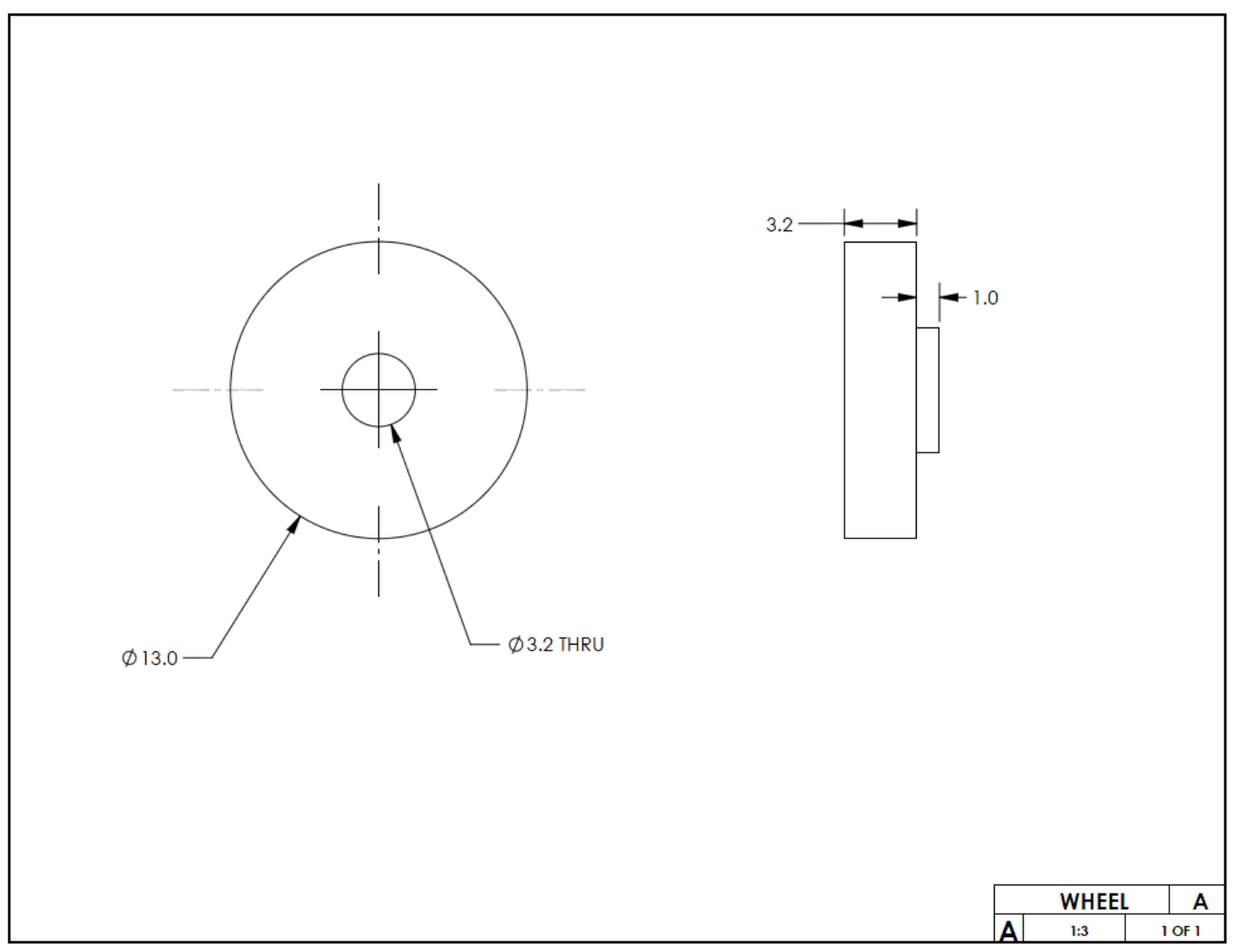




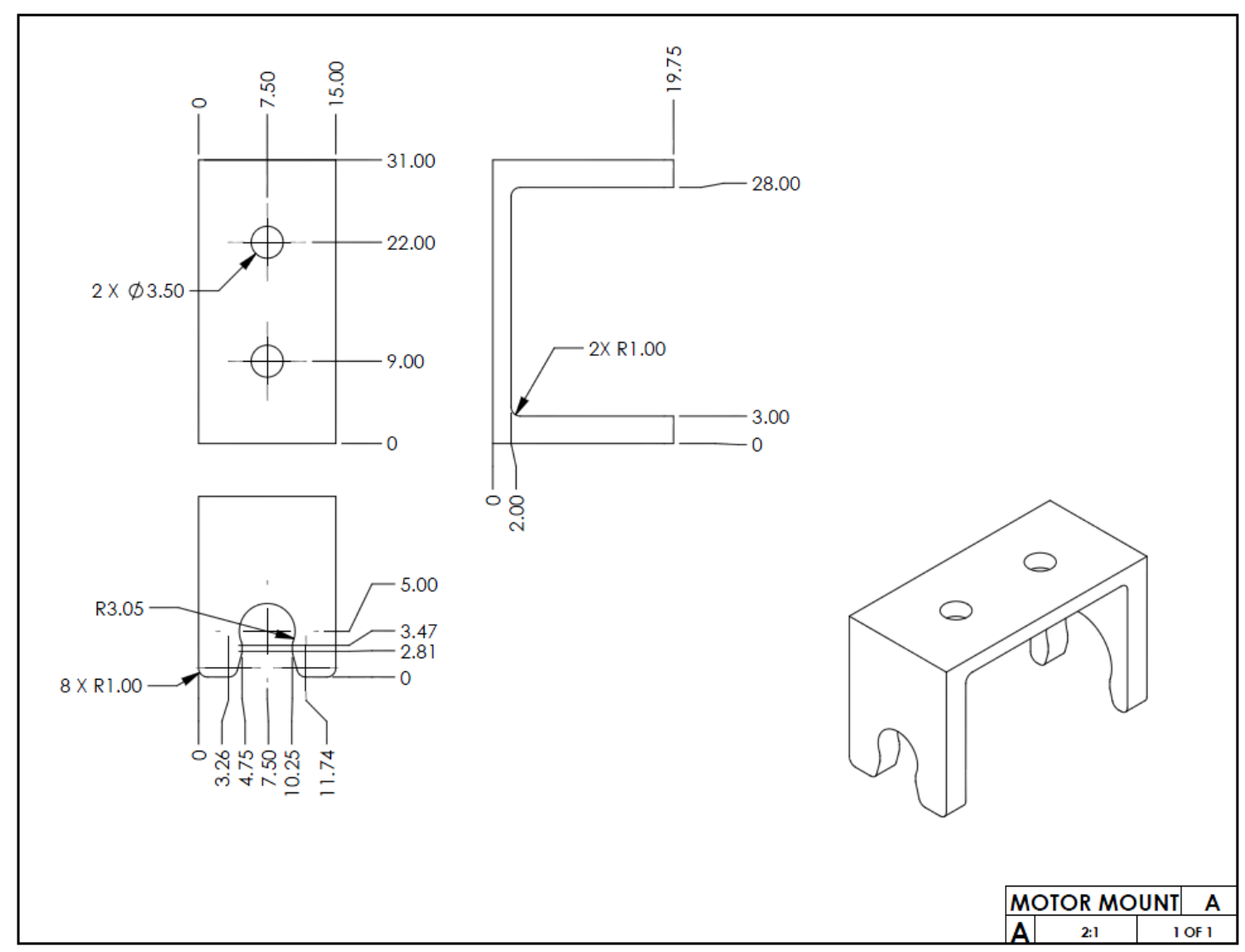




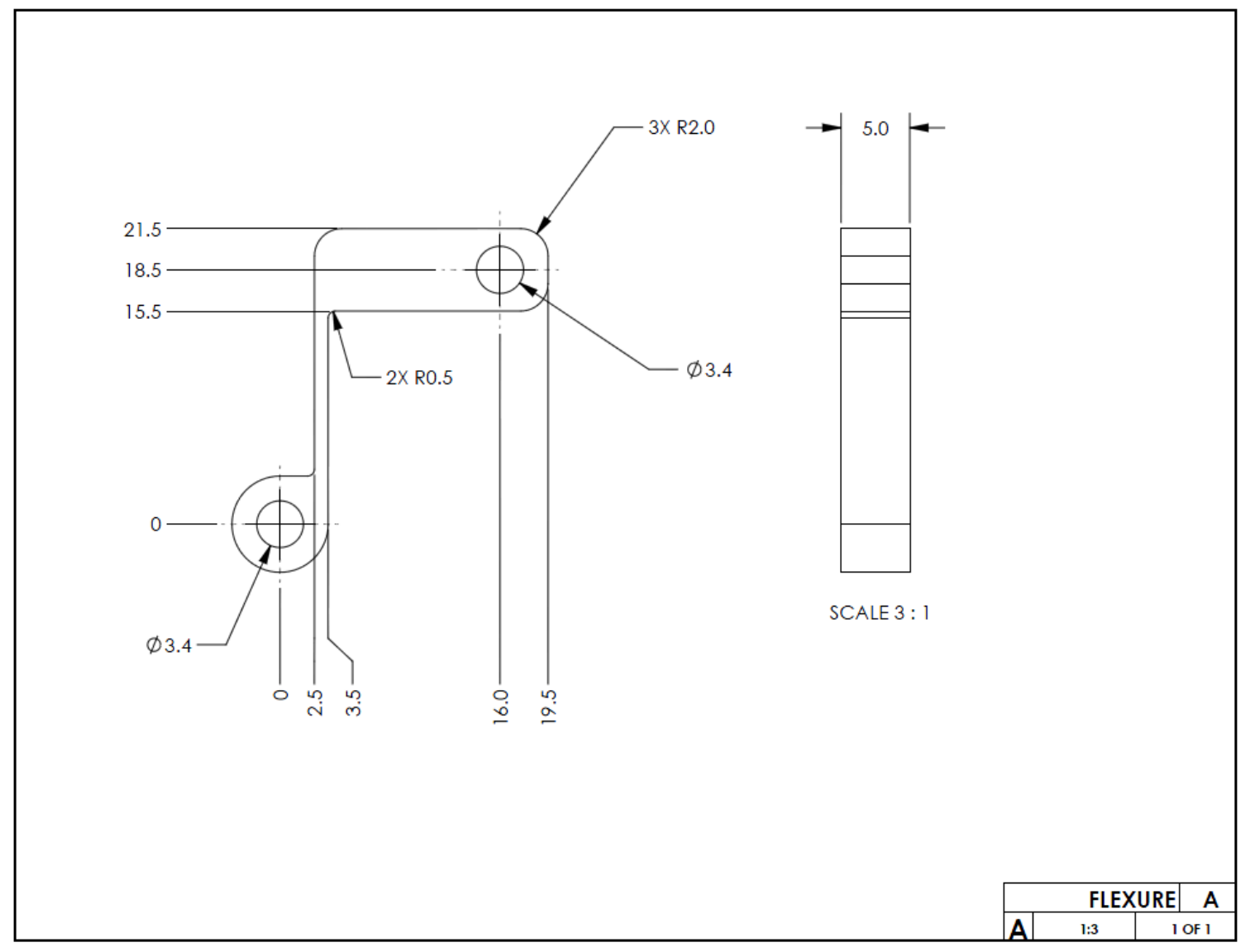




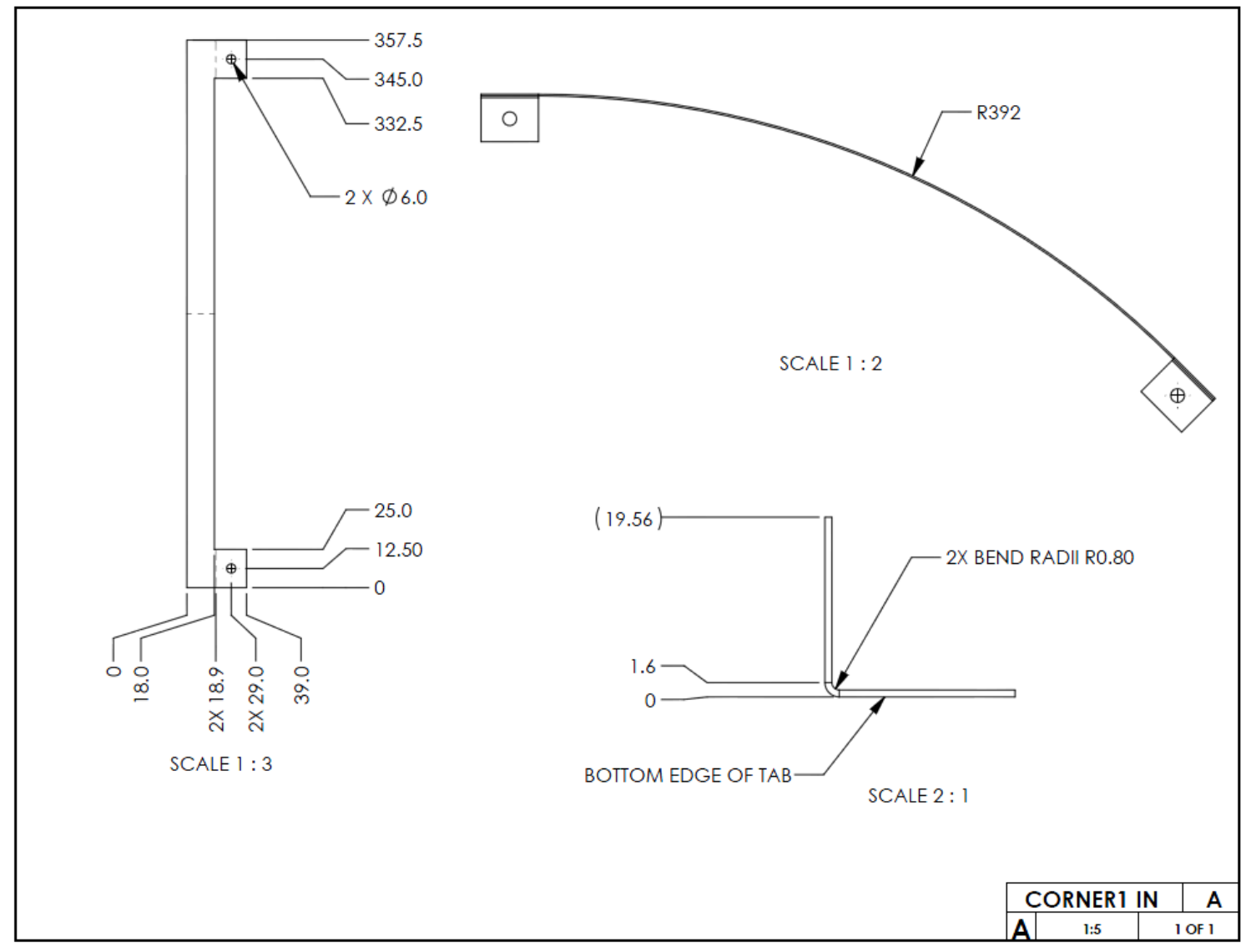




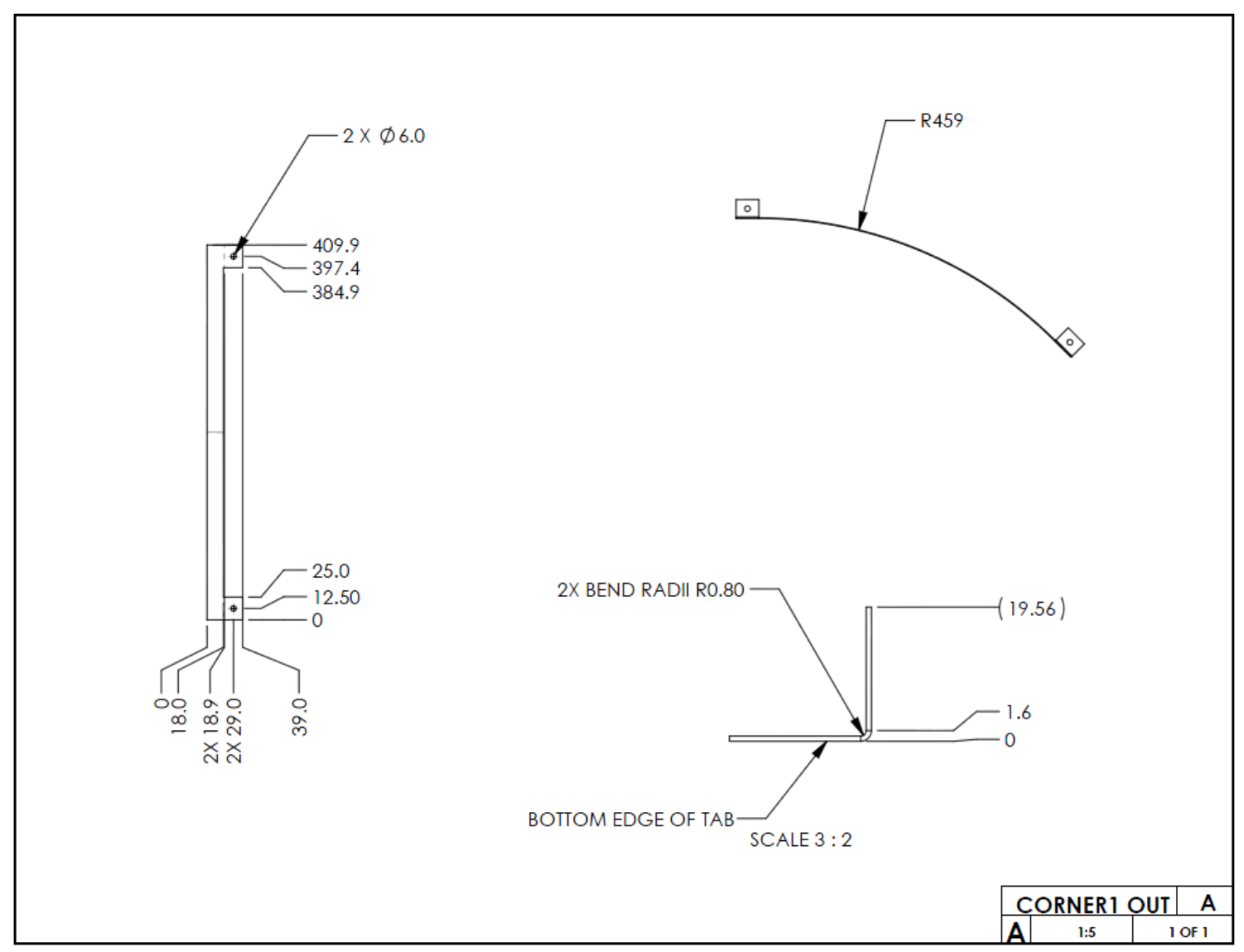




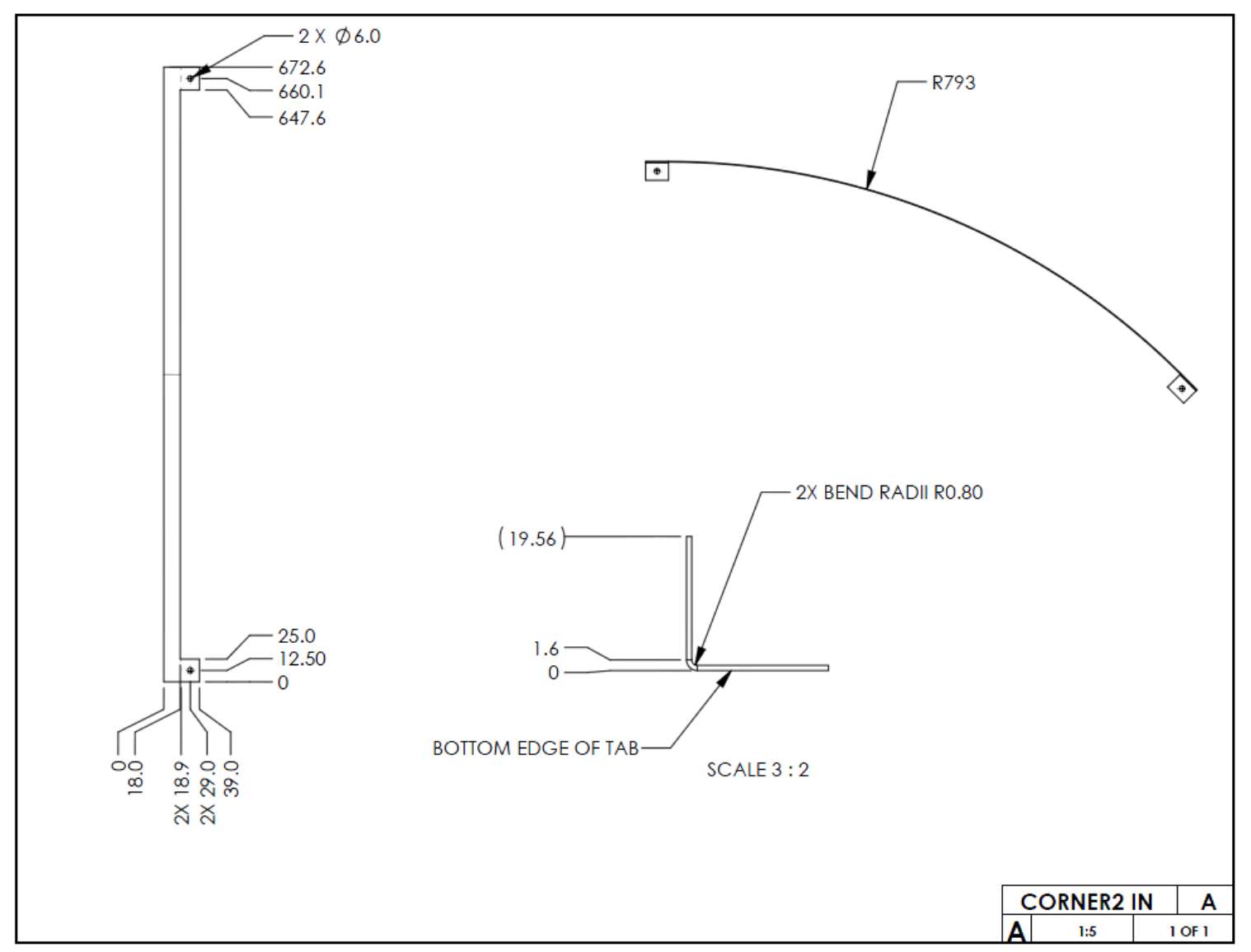




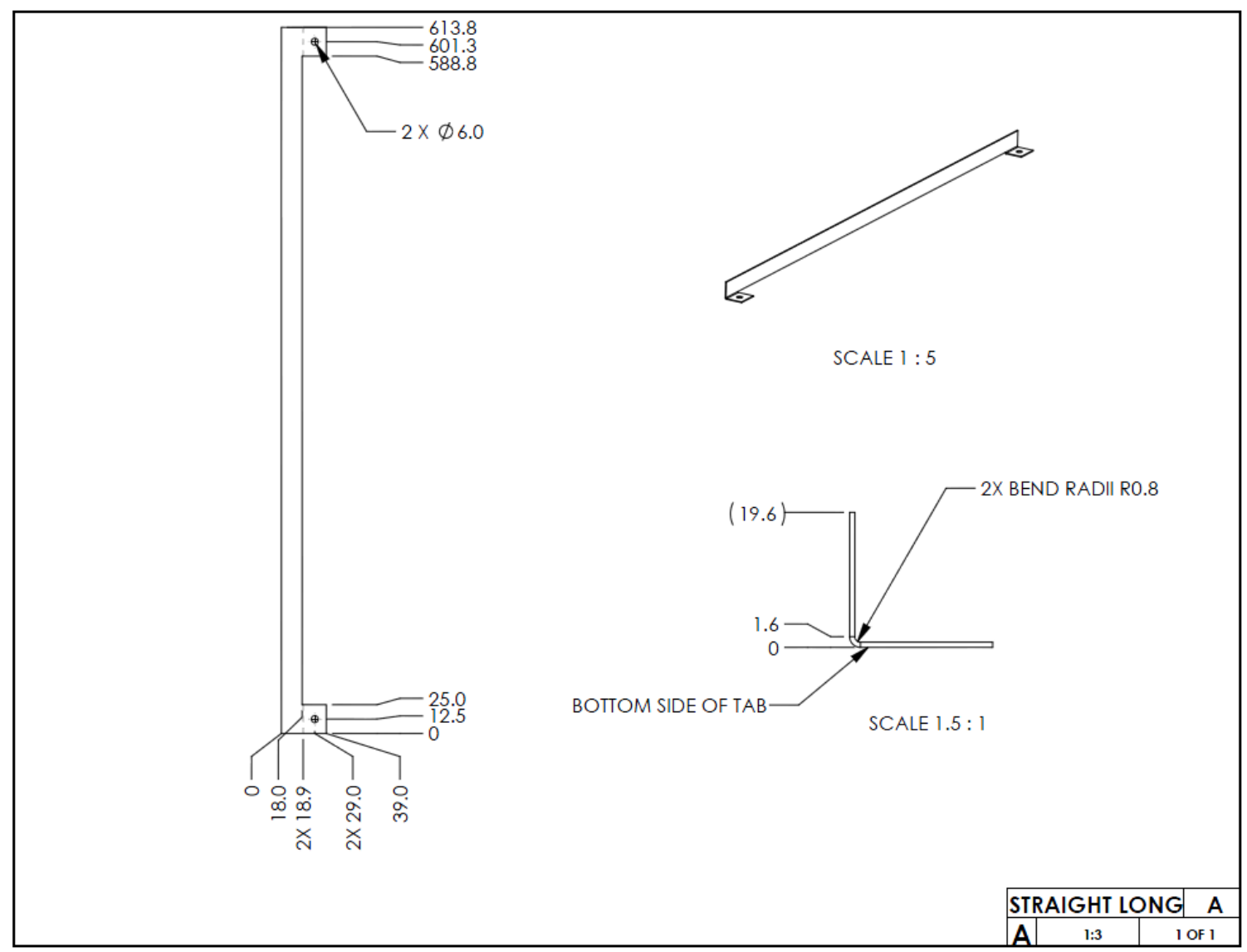




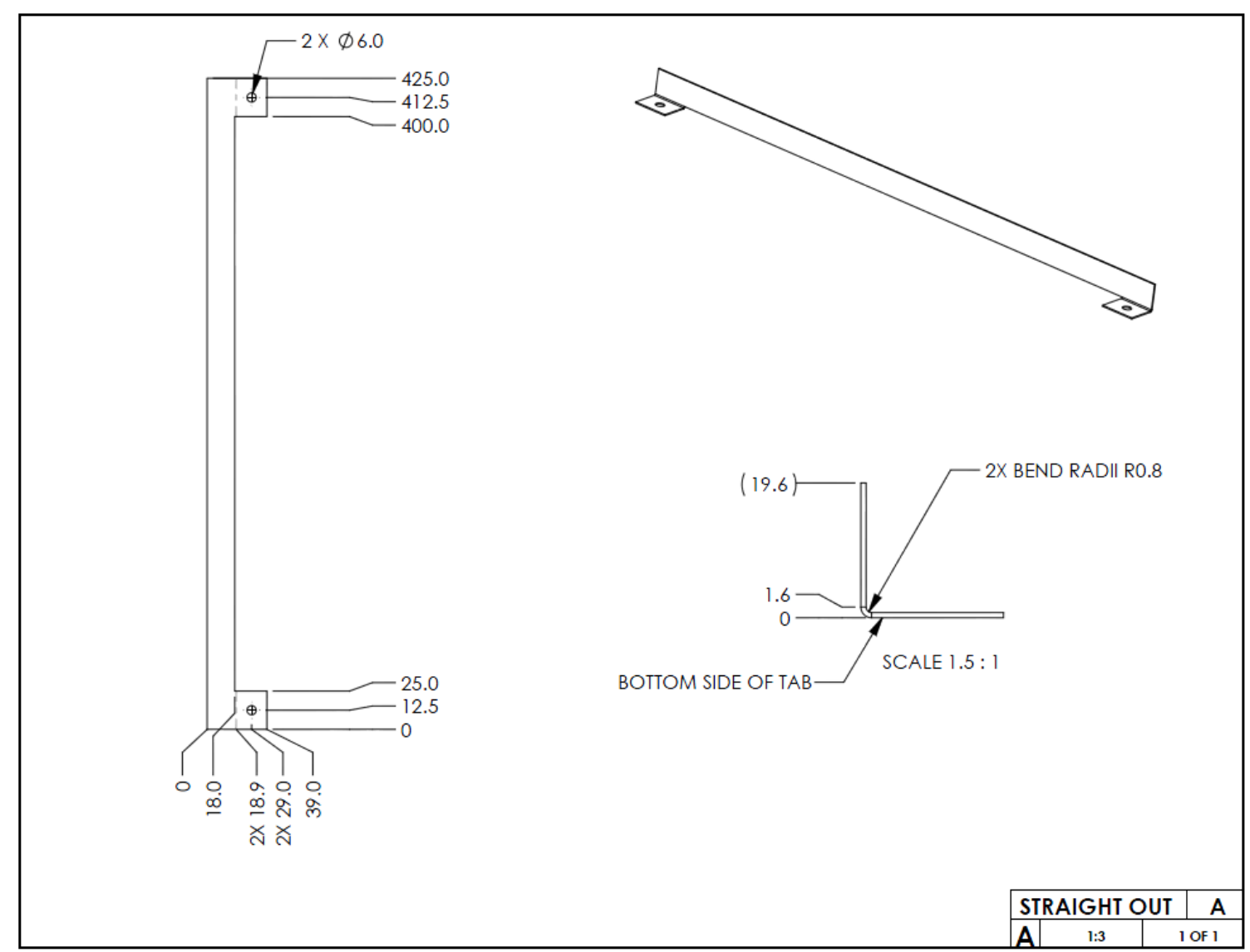




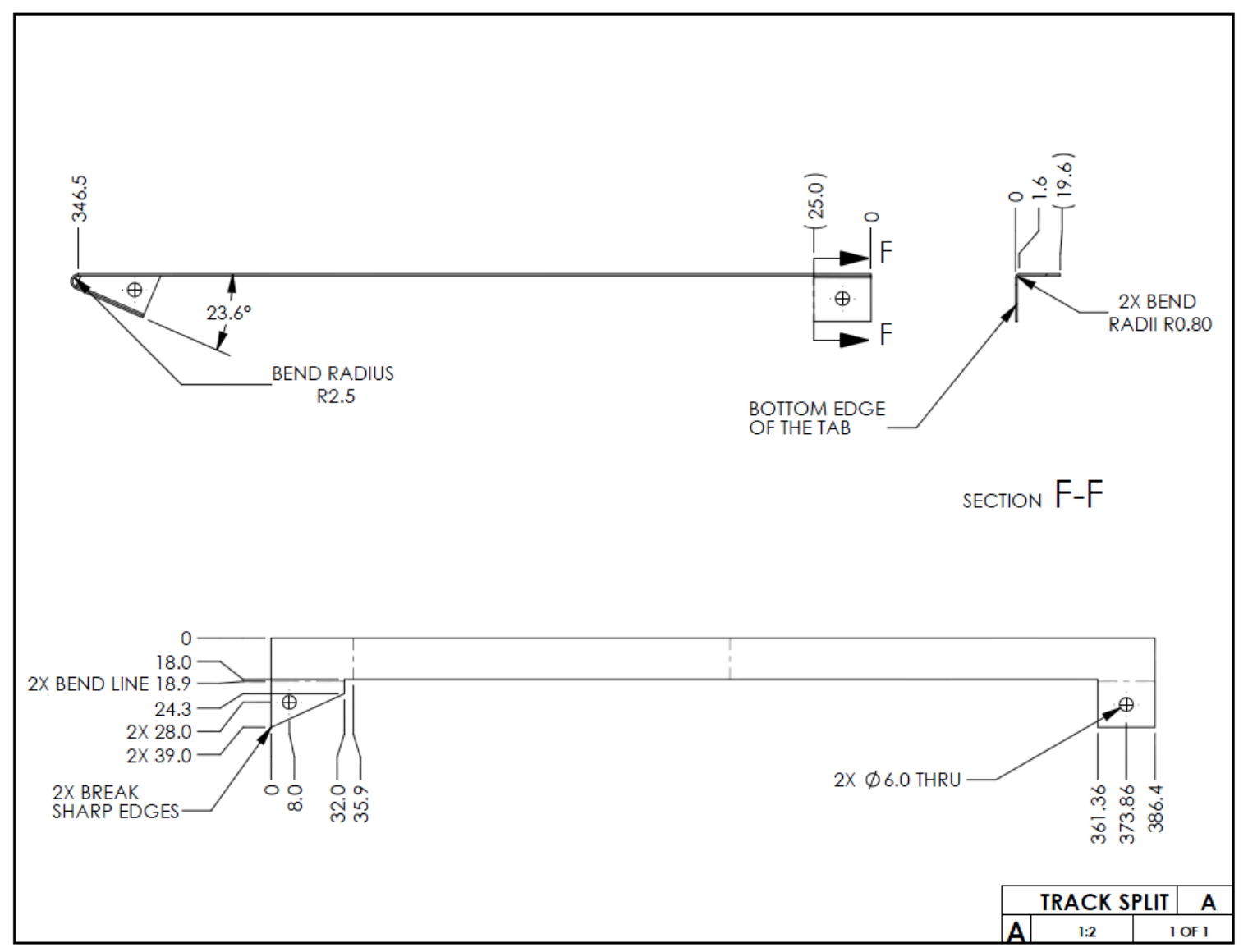




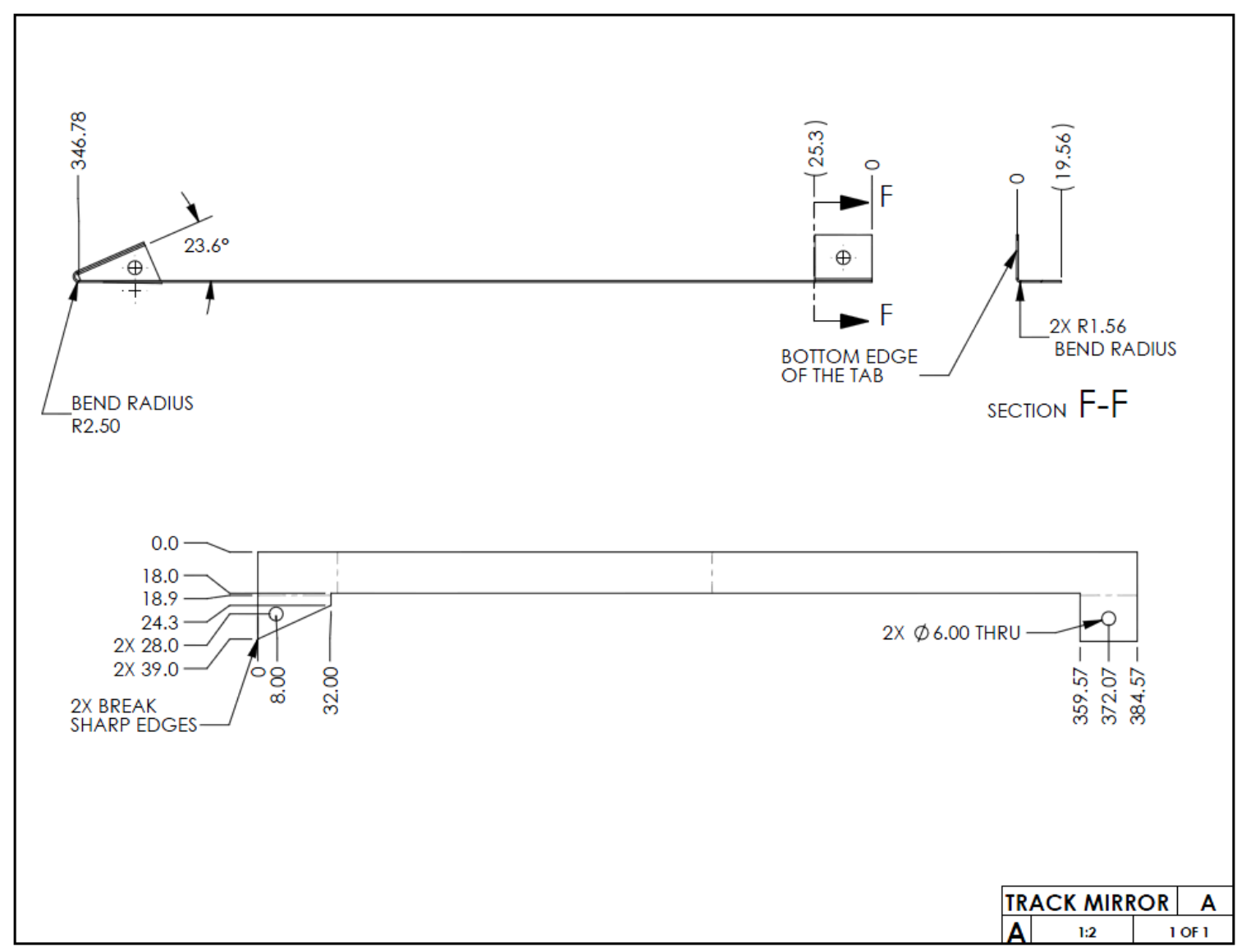




\section{Appendix E}

\section{Matlab Proportional Controller Code}

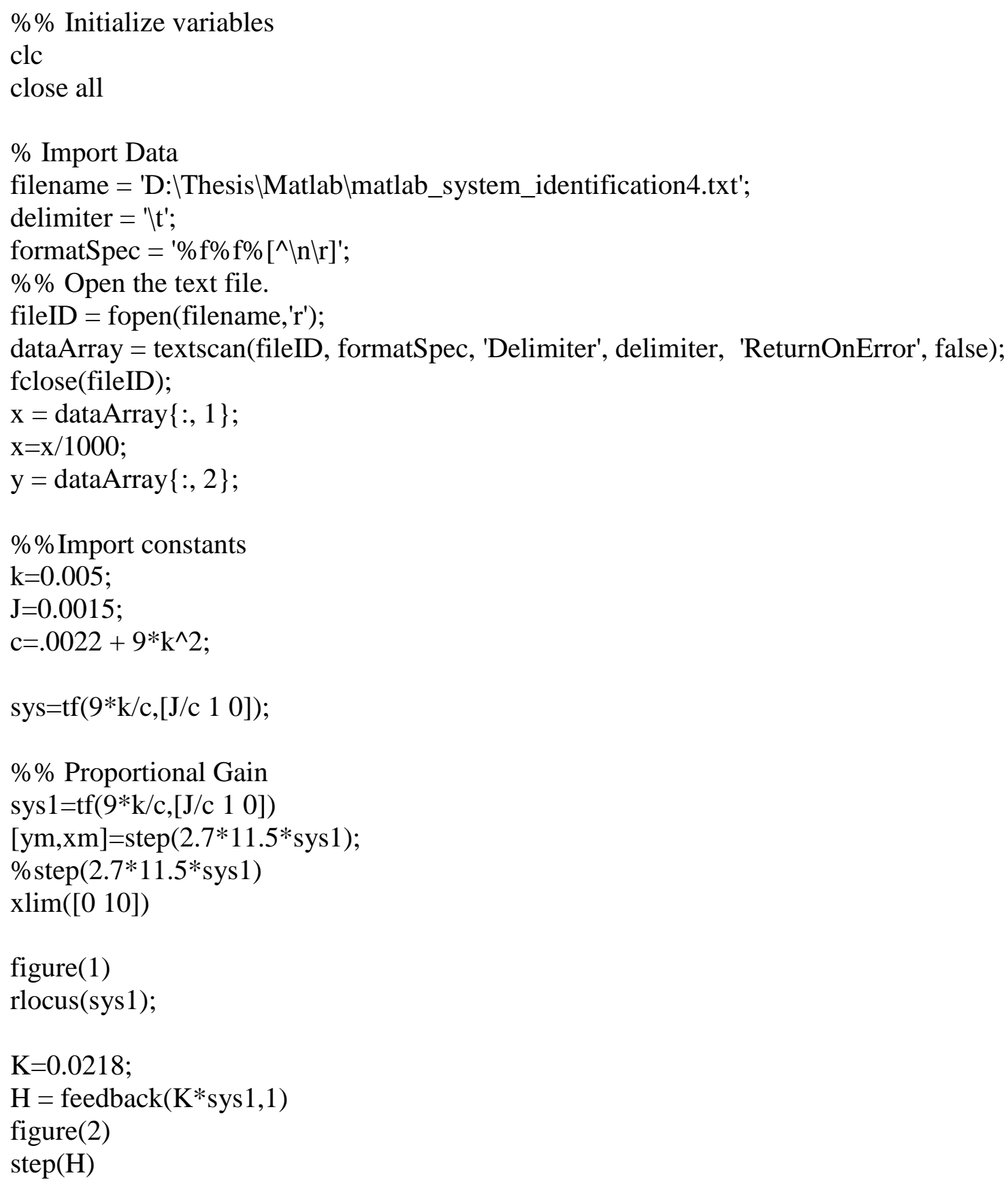




\section{Appendix F}

\section{Matlab Cascaded Controller Code}

$\% \%$ Initialize variables.

filename = 'D: \Thesis \Matlab\matlab_system_identification4.txt';

delimiter $=$ ' $\mid \mathrm{t}$ ';

formatSpec = '\%f\% $\%$ \% [^\} \backslash \mathrm { n } \backslash \mathrm { r } ] \text { '; }

$\% \%$ Open the text file.

fileID = fopen(filename, 'r');

dataArray = textscan(fileID, formatSpec, 'Delimiter', delimiter, 'ReturnOnError', false);

fclose(fileID);

$\mathrm{x}=\operatorname{dataArray}\{:, 1\}$;

$\mathrm{x}=\mathrm{x} / 1000$;

$\mathrm{y}=\operatorname{dataArray}\{:, 2\}$;

$\% \%$ Clear temporary variables

$\mathrm{k}=0.005$;

$\mathrm{J}=0.0015$;

$\mathrm{c}=.0022+9 * \mathrm{k}^{\wedge} 2$

$\% \%$ Cascaded controller

sys $2=\mathrm{tf}(9 * \mathrm{k} / \mathrm{c},[\mathrm{J} / \mathrm{c} 1])$

\%position controller

p_controller $=\operatorname{pid}(1.592, .01)$

\%velocity controller

v_controller $=\operatorname{pid}(0.3,0.01)$

$\mathrm{I}=\mathrm{tf}(1,[1,0]) ; \%$ Integrator

$\mathrm{W}=$ feedback $\left(\mathrm{sys} 2 *{ }^{*} \mathrm{v} \_\right.$controller, 1$)$

figure

$\operatorname{pzplot}(\mathrm{W})$

figure

rlocus (W *p_controller*I)

$\mathrm{W}=$ feedback $\left(\mathrm{W} * \mathrm{p}\right.$ _controller*I* $\left.{ }^{*} 1.96,1\right)$

figure

$\operatorname{step}(\mathrm{W})$

figure

$\operatorname{pzplot}(\mathrm{W})$ 


\section{Appendix G}

\section{Microcontroller Code for the Vehicle}

$1 \quad$ \#include $<$ XBee.h $>$

2

$3 \quad / /$ Include XBee libraries and set up constants

4 extern volatile unsigned long timer0_millis;

$5 \quad / / \mathrm{XBee}$ xbee $=\mathrm{XBee}()$;

$6 \quad$ //ZBRxResponse ZBRx16 = ZBRxResponse();

7 \#define MAX_FRAME_DATA_SIZE 110

8

$9 \quad / / L E D$ in case of errors

10 \#define Err_LED 13

11

//QRE1113 variables

13 \#define Opto_out A2 //Sensor. High when not on Reflector.

14 \#define Opto_in A3//Sensor. High when not on Reflector.

15 \#define Opto_dir A1//Sensor. High when not on Reflector.

17 //Driving Motor Variables

18 \#define EN 4

19 \#define IN1 5

$20 \quad$ \#define IN2 6

21 \#define forwardDuty 100

22 \#define reverseDuty 30

23 \#define FWD $0 / /$ Forward

24 \#define REV $1 / /$ Reverse

25

//Switch Mechanism Variables

\#define SWITCH_TIME 800 //900 ms for switch operation

\#define SWITCH_REST_TIME 2000 // $2000 \mathrm{~ms}$ for switch in a position \#define SWITCH_RETURN_TIME 2800

\#define switch_upDuty 50

//right-hand side motor-controlled switch

\#define EN_RSwitch 12

\#define IN1_RSwitch 7

\#define IN2_RSwitch 11

//left-hand side motor-controlled switch

\#define EN_LSwitch 12

\#define IN1_LSwitch 3

\#define IN2_LSwitch 11

40 


\begin{tabular}{|c|c|}
\hline 41 & //PID variables \\
\hline 42 & \#define kp 0.3 \\
\hline 43 & \#define ki 0.01 \\
\hline 44 & \#define kv 1.592 \\
\hline 45 & \#define kvd 0 \\
\hline 46 & \#define kvi 0.01 \\
\hline 47 & \\
\hline 48 & //time-counter variables \\
\hline 49 & long unsigned time_check; \\
\hline 50 & \\
\hline 51 & //DEFINE MORE VARIABLES \\
\hline 52 & int o_sensor_in $=0$; \\
\hline 53 & int o_sensor_out $=0$; \\
\hline 54 & int o_sensor_dir $=0$; \\
\hline 55 & long unsigned time_now $=0$; \\
\hline 56 & long unsigned time_last $=0$; \\
\hline 57 & long unsigned time_last_line $=0$; \\
\hline 58 & long unsigned time_line $=0$; \\
\hline 59 & long unsigned switch_start $=0$; \\
\hline 60 & long unsigned time_dir $=0$ \\
\hline 61 & int change $=0$ \\
\hline 62 & int count_sensor $=0$; \\
\hline 63 & long location=-180; \\
\hline 64 & byte checkpoint $=0$ \\
\hline 65 & long velocity $=0$ \\
\hline 66 & long vel=0; \\
\hline 67 & long vel2=0; \\
\hline 68 & long vel3=0; \\
\hline 69 & int proximity $=0$ \\
\hline 70 & int $\operatorname{count}=1$ \\
\hline 71 & int count_pos $=1$; \\
\hline 72 & int count_vel = 1 ; \\
\hline 73 & int white $=0$ \\
\hline 74 & int white_dir $=0$; \\
\hline 75 & int spillover $=0$ \\
\hline 76 & boolean rest $=0$; \\
\hline 77 & int pause $=0$ \\
\hline 78 & int pos_check $=0$; \\
\hline 79 & int stoptime $=130 * 2$ \\
\hline 80 & boolean switch_finished_left $=0$; \\
\hline 81 & boolean switch_finished_right $=0$ \\
\hline 82 & int pos_error $=0$ \\
\hline 83 & int vel_error=0; \\
\hline 84 & int last_pos_location $=0$; \\
\hline
\end{tabular}




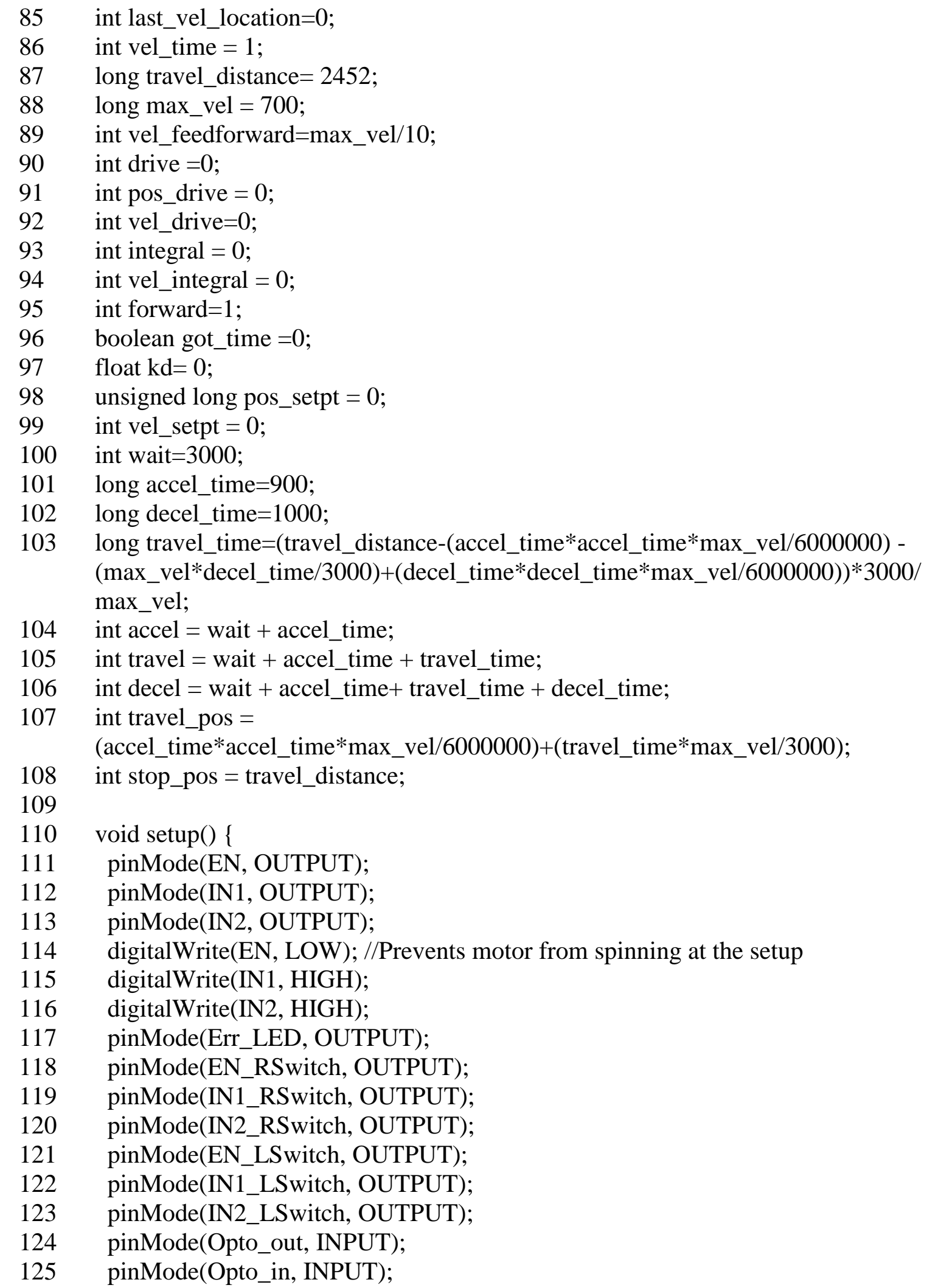


126 pinMode(Opto_dir, INPUT);

127 pinMode(prox, INPUT);

128 //SwitchesReset();

129 pinMode(13, OUTPUT);

130

131

132

133

134

135

136

137

138

139

140

141

142

143

144

145

146

147

148

149

150

151

152

153

154 void $\operatorname{loop}()\{$

155 // PID Loop for Velocity

156 if $($ millis ()$/$ count_vel $>=50)\{$ //Run loop every $2 \mathrm{~ms}$

157

158

159

Serial.begin(115200);

Serial.flush();

xbee.begin(115200);

//Get start command

$$
\text { while (got_time }==0)\{
$$

xbee.readPacket();

if (xbee.getResponse().isAvailable()) \{

// got something

xbee.getResponse().getZBRxResponse(ZBRx16);

Serial.print(ZBRx16.getData(0));

if $($ ZBRx16.getData(0) $==99$ \&\& ZBRx16.getData(1) == 99 \&\&

ZBRx 16.getData(2) ==99) \{ setMillis(0);

160 vel_setpt=(millis ()$-$ wait $) * \max \_v e l / 1000$;

$161\}$

162 else if $($ millis ()$<$ travel $)\{$

163 vel_setpt=max_vel;

$164\}$

165 else if $($ millis ()$<$ decel $)\{$

166 vel_setpt=max_vel-max_vel*(millis ()$-$ travel $) / 1000$;

$167\}$

168 else \{ 


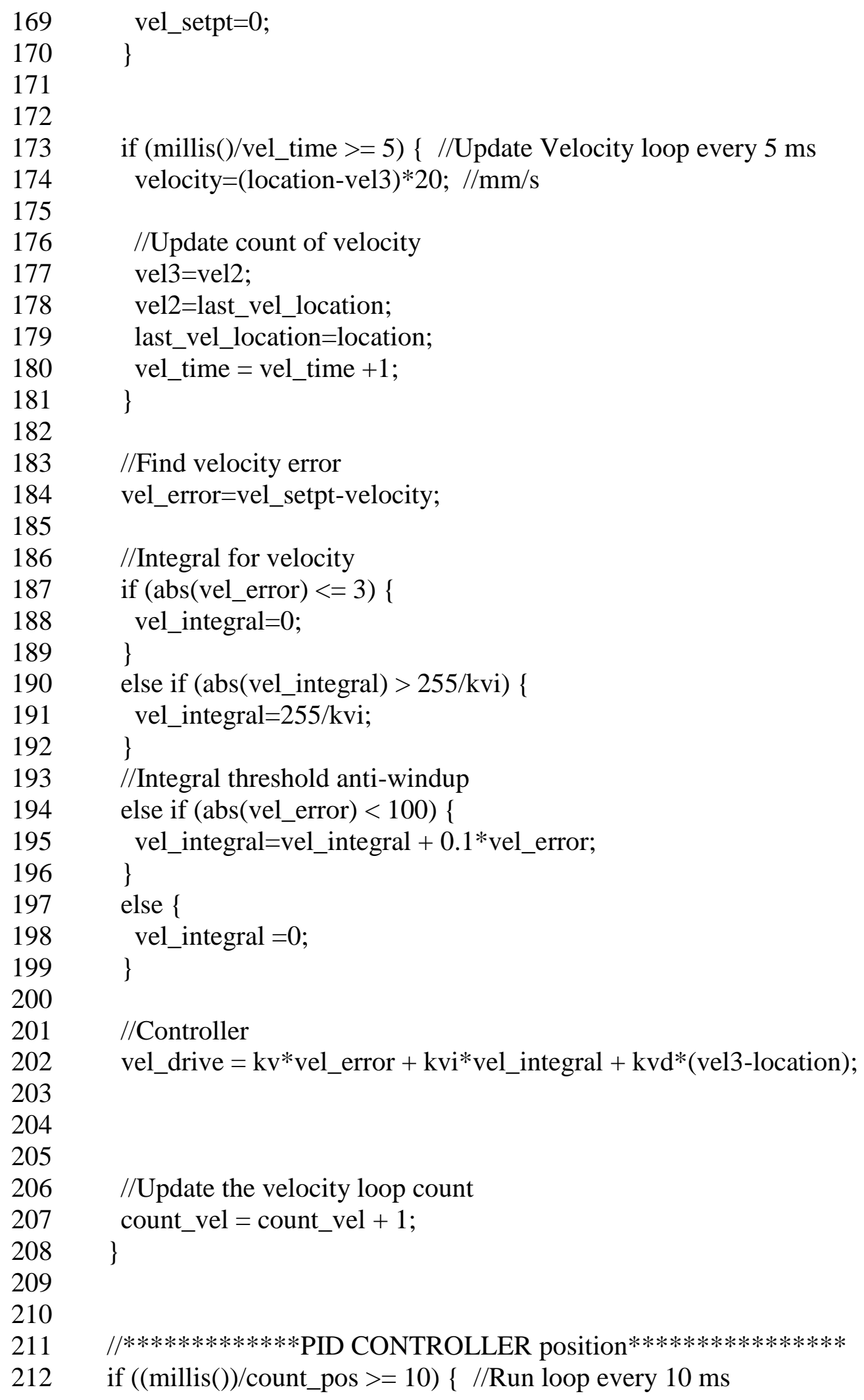




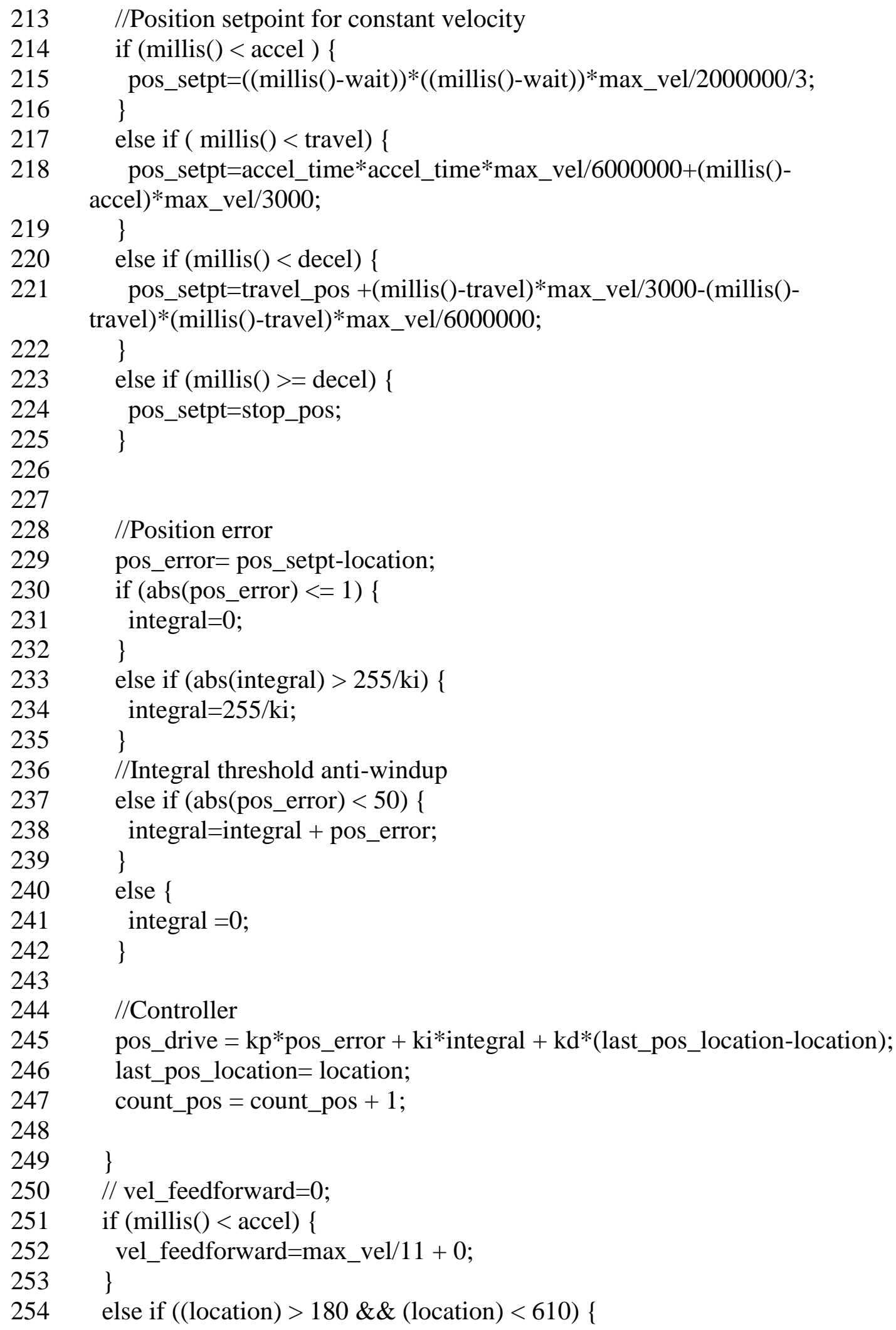




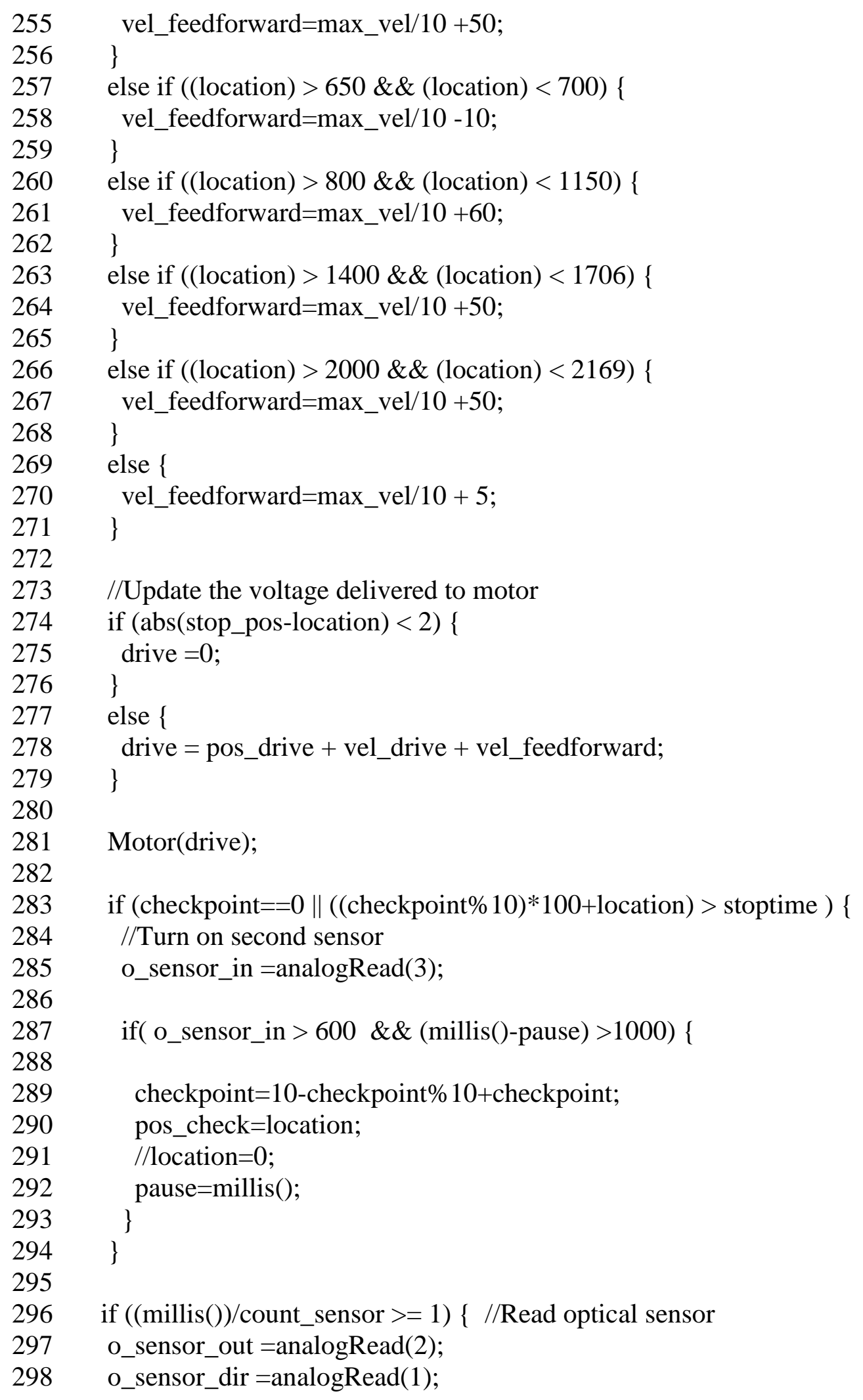


if $($ o_sensor_out $>470 \& \&$ white $==1)\{$

303

304 


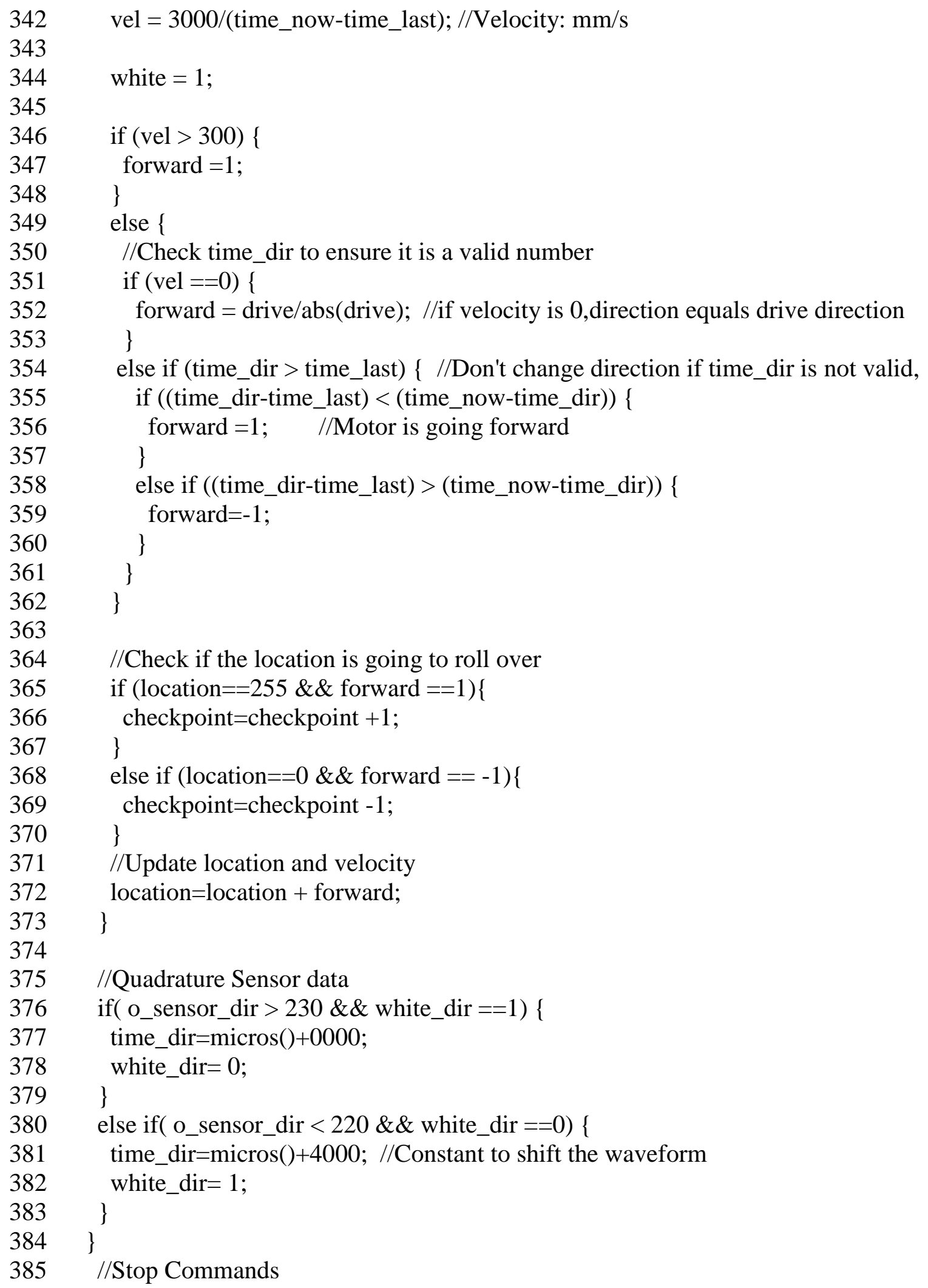


386 if $($ checkpoint $==50 \& \&$ switch_finished_left $==0) \quad\{$

387 if (switch_start $==0)\{$

388 switch_start=millis () ;

$389\}$

390

391 if (millis()-switch_start < 35) \{

$392 \quad$ Switch(1);

$393\}$

394 else \{

$395 \quad$ Switch(0);

396 switch_finished_left=1;

$397 \quad$ stoptime $=160 * 2$;

398 switch_start $=0$;

$399\}$

$400\}$

401

402 if (checkpoint $==20 \& \&$ switch_finished_right $==0) \quad\{\quad / / 200$ inside $/ / 229$ outside

403 if (switch_start $==0) \quad\{$

404 switch_start=millis();

$405\}$

406

407 if (millis () -switch_start < 35) \{

$408 \quad$ //Switch(2);

$409\}$

$410 \quad$ else \{

411 Switch(0);

412 switch_finished_right $=1$;

$413 \quad$ stoptime $=130$;

414 switch_start $=0$;

$415\}$

416

$417 \quad \operatorname{Motor}(0)$;

$418\}$

419

$420 \quad / /$ Transmit Data over XBee

421 if $((\operatorname{millis}()) /$ count $>49||$ pos_check !=0) \{

422

423

424

425

426

427

428

$$
\text { uint8_t payload[] }=\{
$$

pos_setpt,(location/100)\%100,(location)\%100, abs(vel_integral), vel_setpt/10,velocity/10, drive

;

//Address

XBeeAddress64 addr64_c = XBeeAddress64(0x0013A200, 0x40ABB737); 


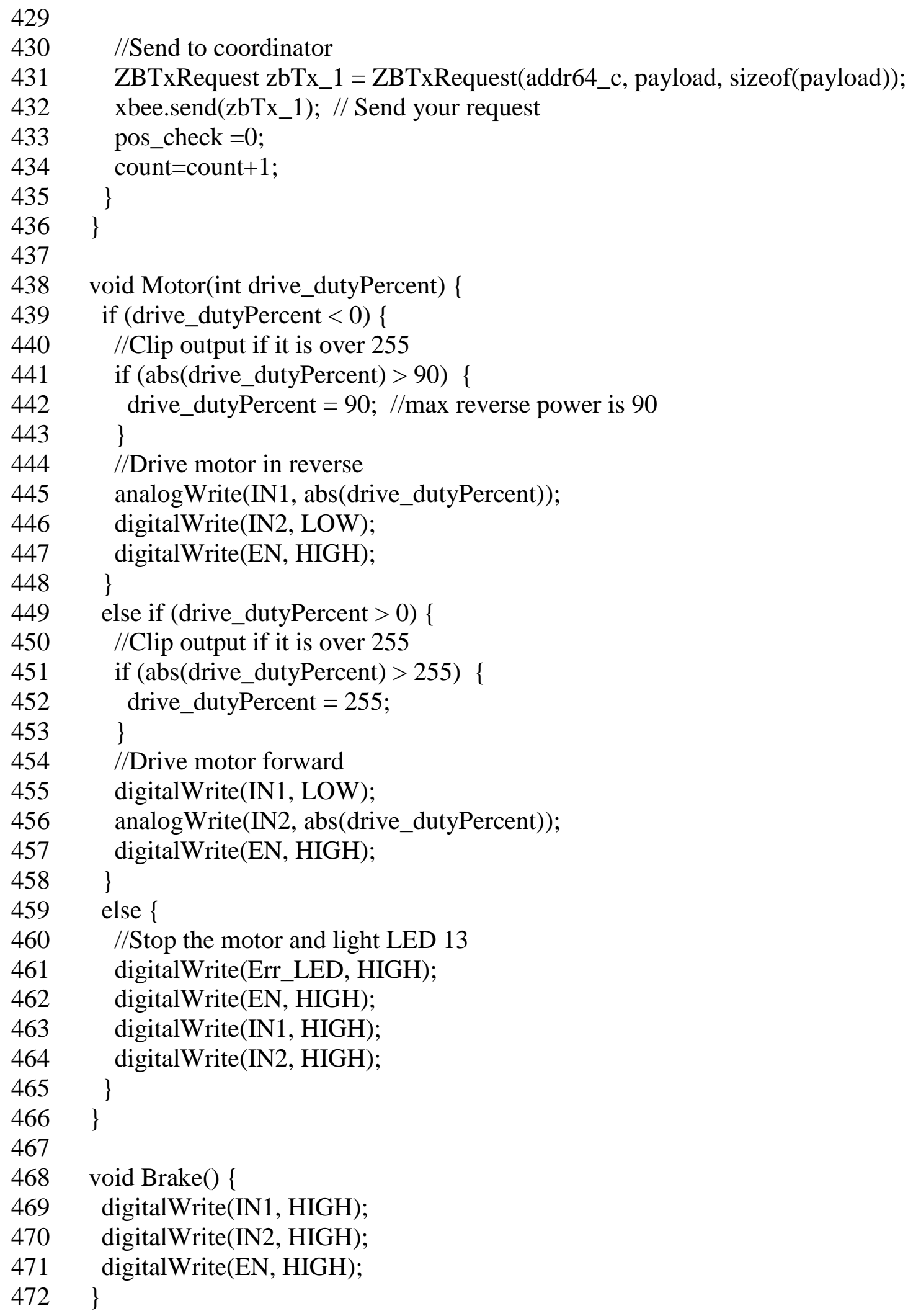


474 void Switch(int switch_direction) \{

475 if (switch_direction == 1) \{ // left switch down

476 analogWrite(IN1_RSwitch, 235);

477 digitalWrite(IN2_RSwitch, LOW);

478 digitalWrite(EN_RSwitch, HIGH);

$479 \quad\}$

480 if (switch_direction $==2)\{$ //right switch down

481 analogWrite(IN2_RSwitch, 235);

482 digitalWrite(IN1_RSwitch, LOW);

483 digitalWrite(EN_RSwitch, HIGH);

$484 \quad\}$

485 if (switch_direction $==0)\{$ //Stops the switch

486 digitalWrite(IN1_RSwitch, HIGH);

487 digitalWrite(IN2_RSwitch, HIGH);

488 digitalWrite(EN_RSwitch, HIGH);

$489 \quad\}$

$490 \quad\}$

491

492 void setMillis(unsigned long new_millis)\{

493 uint8_t oldSREG = SREG;

$494 \quad \operatorname{cli}() ;$

495 timer0_millis = new_millis;

$496 \quad$ SREG = oldSREG;

$497 \quad\}$ 